\title{
THREE DIMENSIONAL SIMULATION OF RAYLEIGH-BÉNARD CONVECTION FOR RAPID MICROSCALE POLYMERASE CHAIN REACTION
}

\author{
A Dissertation \\ by \\ RADHA MALINI GOWRI MUDDU \\ Submitted to the Office of Graduate Studies of \\ Texas A\&M University \\ in partial fulfillment of the requirements for the degree of \\ DOCTOR OF PHILOSOPHY
}

December 2010

Major Subject: Mechanical Engineering 
Three Dimensional Simulation of Rayleigh-Bénard Convection for Rapid Microscale Polymerase Chain Reaction Copyright 2010 Radha Malini Gowri Muddu 


\title{
THREE DIMENSIONAL SIMULATION OF RAYLEIGH-BÉNARD CONVECTION FOR RAPID MICROSCALE POLYMERASE CHAIN REACTION
}

\author{
A Dissertation \\ by \\ RADHA MALINI GOWRI MUDDU
}

\author{
Submitted to the Office of Graduate Studies of \\ Texas A\&M University \\ in partial fulfillment of the requirements for the degree of \\ DOCTOR OF PHILOSOPHY
}

\begin{abstract}
Approved by:
Co-Chairs of Committee, Yassin A. Hassan

Victor M. Ugaz

Committee Members, William Marlow

H.C. Chen

Head of Department, Dennis O’Neal
\end{abstract}

December 2010

Major Subject: Mechanical Engineering 


\author{
ABSTRACT \\ Three Dimensional Simulation of Rayleigh-Bénard Convection for Rapid Microscale \\ Polymerase Chain Reaction. (December 2010) \\ Radha Malini Gowri Muddu, B.Tech.; M.Tech., Indian Institute of Technology, Madras; \\ M.S., Purdue University \\ Co-Chairs of Advisory Committee: Dr. Yassin A. Hassan \\ Dr. Victor M. Ugaz
}

Rayleigh-Bénard convection has been extensively studied in literature owing to its ubiquitous nature. However, most of the studies have been confined to geometries where the aspect ratio of the cylinder was less than 1 . Here we study the motion of fluid in geometries with aspect ratio greater than 1, with particular application to use of such motion to actuate biochemical reactions, such as the polymerase chain reaction. We show that it is possible to accelerate the rate of reaction by using a geometry that promotes chaotic motion versus a geometry that promotes quasi- periodic motion. We also simulate chemical kinetics using the fluid motion as a starting point and we prove that chaotic motion indeed enhances the rate of the reaction. We also provide qualitative and quantitative measures for chaotic motion in a fluid flow, which helps to distinguish between different types of fluid motion. We highlight the transitions between different types of flow that are possible with Rayleigh-Bénard convection. Finally, we compare our simulations against experimental data obtained from particle image velocimetry, laser induced fluorescence and optical microscopic visualization. 


\section{DEDICATION}

To Mum, Dad, Raj, Priya, Meenakshi, Madhuri and, of course, Akhilesh 


\section{ACKNOWLEDGEMENTS}

First, I would like to acknowledge my advisors, Prof. Yassin A. Hassan and Prof. Victor M. Ugaz, for their guidance through my Ph.D. and for involving me in a variety of challenging projects. They have been a source of constant support and inspiration during my course of study - my work has only been possible because of their dedication and pursuit of excellence.

I am also indebted to all my colleagues at the Ugaz and Hassan lab - Tony, Wendy, Nan, Serder, Airong, Winnie, Clemente, Hugo, Ashish, Carlos Estrada-Perez, Noushin and Dr. Tan.

I would also like to thank my parents and my husband, Akhilesh, for inspiring me to go for a Ph.D. 


\section{NOMENCLATURE}

\begin{tabular}{|c|c|}
\hline$c_{i}$ & dimensionless concentration \\
\hline$c^{*}$ & characteristic concentration constant \\
\hline$c_{i}^{\prime}$ & dimensional concentration \\
\hline$c_{d s}$ & dimensionless concentration of dsDNA \\
\hline$c_{s S}$ & dimensionless concentration of ssDNA \\
\hline$c_{a}$ & dimensionless concentration of aDNA \\
\hline$C^{d}$ & correlation sum \\
\hline$d$ & number of embedding dimensions \\
\hline$D$ & correlation dimension \\
\hline$d_{p}$ & diameter of particle \\
\hline$D_{P}$ & characteristic dimension of particle \\
\hline$d$ & diameter of cylinder \\
\hline$D$ & mass diffusion coefficient \\
\hline$D a^{c}$ & convective Damköhler number \\
\hline $\mathbf{e}_{\mathbf{z}}$ & unit vector in the vertically downward direction \\
\hline$f_{d s}$ & mapping function for dsDNA \\
\hline$f_{s s}$ & mapping function for ssDNA \\
\hline$f_{a}$ & mapping function for aDNA \\
\hline$g$ & acceration due to gravity \\
\hline$h$ & height of the cylinder \\
\hline
\end{tabular}




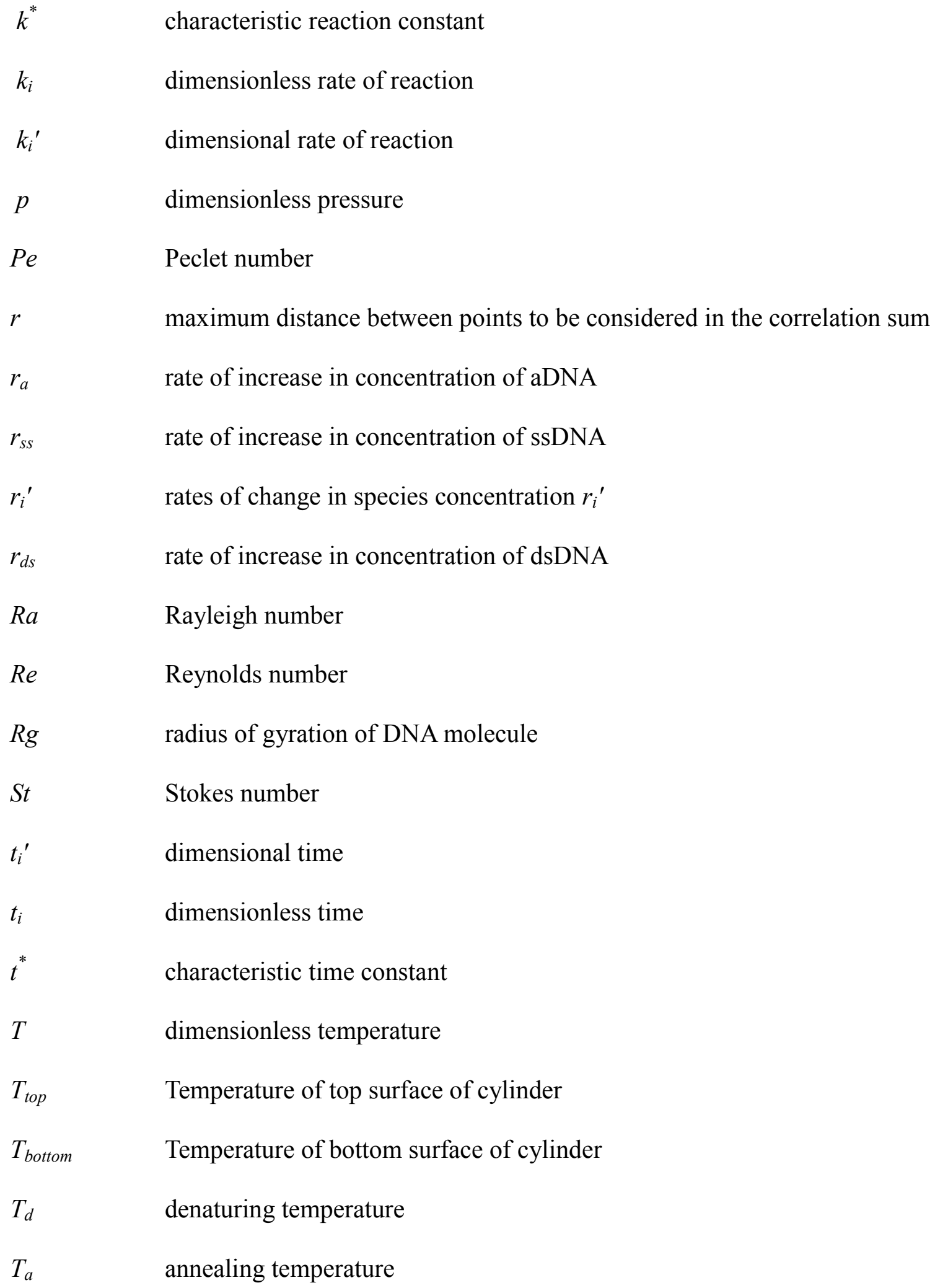




\begin{tabular}{|c|c|}
\hline$T_{e}$ & extension temperature \\
\hline$\Delta T$ & Temperature difference \\
\hline $\bar{u}$ & velocity averaged over the flow trajectory \\
\hline$U$ & average velocity of flow \\
\hline $\boldsymbol{V}$ & dimensionless velocity vector \\
\hline$\alpha$ & Thermal diffusivity \\
\hline$\beta$ & coefficient of thermal expansion of fluid \\
\hline$\eta$ & suspension viscosity \\
\hline$\eta_{o}$ & solvent viscosity \\
\hline$\theta$ & dimensionless temperature \\
\hline$\Theta$ & Heaviside step function \\
\hline$\kappa_{\mathrm{ds}}$ & dimensionless rate of reaction of dsDNA \\
\hline$\kappa_{\mathrm{ss}}$ & dimensionless rate of reaction of ssDNA \\
\hline$\kappa_{\mathrm{a}}$ & dimensionless rate of reaction of Adna \\
\hline$v$ & kinematic viscosity \\
\hline$\sigma_{i}$ & standard deviation of reaction rate \\
\hline$\tau$ & doubling time \\
\hline$\varnothing$ & volume fraction \\
\hline
\end{tabular}




\section{TABLE OF CONTENTS}

\section{Page}

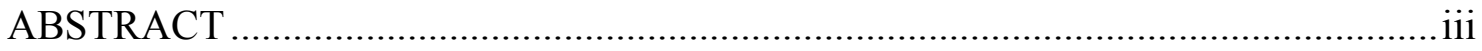

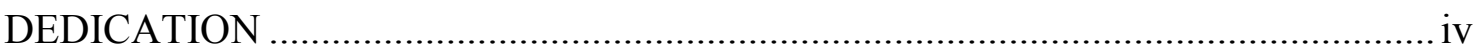

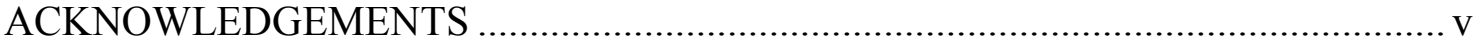

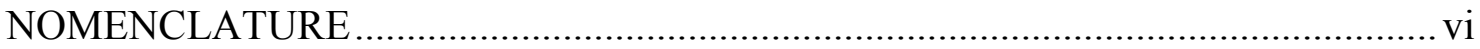

TABLE OF CONTENTS ............................................................................. ix

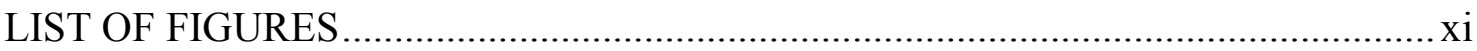

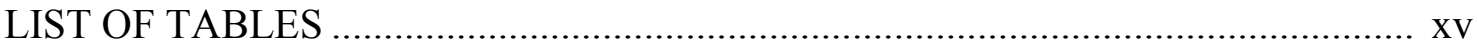

CHAPTER

I INTRODUCTION: NATURAL CONVECTION IN BIOCHEMICAL REACTIONS ............................................................................. 1

Rayleigh-Bénard convection .............................................. 1

Polymerase chain reaction....................................................... 3

Natural convection based polymerase chain reaction ................. 7

Other convective devices in literature ..................................... 11

II COMPUTATIONAL FLUID DYNAMICS SIMULATION .............. 15

Grid convergence ............................................................... 18

Numerical results of simulations ............................................ 19

Two dimensional simulations of PCR geometries ..................... 24

Experiments in redesigned reactor .......................................... 25

Computational fluid dynamics simulations of PCR geometries .... 30

III NUMERICAL SIMULATION OF CHEMICAL REACTIONS ......... 34

Introduction ............................................................................ 34

Coupled flow and chemical reactions ........................................ $\quad 35$

Evaluating the kinetic model ...................................................... 39

Results of chemical reaction kinetics ........................................ 41 
CHAPTER $\quad$ Page

IV QUANITFYING CHAOS IN CONVECTIVE FLOWS ................. 46

Introduction ................................................................ 46

Poincaré sections ................................................................ 47

Correlation dimension .......................................................... 49

Transitions between flows..................................................... 57

V FLOW MEASUREMENT TECHNIQUES ................................. 67

Introduction to Particle Image Velocimetry ................................. 67

Experimental setup for PIV ..................................................... 69

Results of PIV experiments ................................................. 75

Comparison of numerical results with PIV experiment .............. 76

Introduction to Laser Induced Flourescence ............................ $\quad 79$

Experimental setup for Laser Induced Flourescence .................. $\quad 80$

Results from LIF experiment .............................................. 85

Numerical simulations of LIF experiment .............................. 87

Visualization using optical microscope................................... 88

VI CONCLUSIONS ................................................................. 92

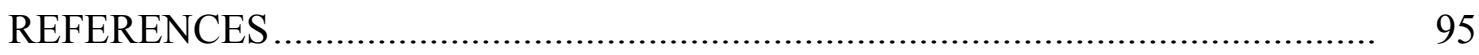

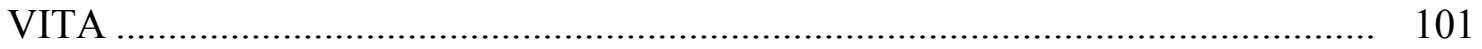




\section{LIST OF FIGURES}

FIGURE

Page

1 Schematic of Rayleigh-Bénard convection in a cylinder ...................... 1

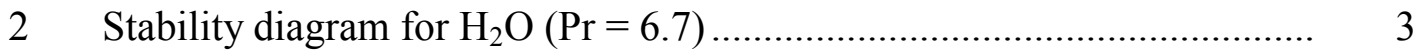

3 Schematic of the process of polymerase chain reaction showing the various steps involved in a cycle ........................................................ 5

4 Commercial bench top PCR instrumentation...................................... 6

5 Prototype of cavity-based convective PCR thermocycling device incorporating a Plexiglas PCR cartridge sandwiched between a top plate connected to a circulating water bath and a bottom plate incorporating cartridge heaters

6 Schematic of the process of gel electrophoresis.

7 Comparison of products obtained from a RB-PCR system with conventional thermocycler (all reactions run for $40 \mathrm{~min}$.)

8 Comparison of reaction times in the RB-PCR device.

9 Spectrum of Rayleigh numbers vs. aspect ratio (h/d) for different reaction volumes

10 Grid convergence study showing the magnitudes of velocity along a line drawn at a circular section which bisects the cylinder into two equal parts

11 Contour plots of temperature and velocity in the $\mathrm{XZ}$ and $\mathrm{YZ}$ planes for cylinder with aspect ratio 3

12 Vector plots of velocity in the $\mathrm{XZ}$ and $\mathrm{YZ}$ planes for cylinders with aspect ratio 8 and 3 , respectively

13 Schematic of points chosen as starting points for extracting streamtraces

14 Particle paths in the XZ, YZ and XY planes for cylinders with aspect ratio 8 , respectively 
15 Contour plots of velocity and temperature for two dimensional simulation of cylinder with aspect ratio 3

16 Results from the cylinder with aspect ratio 3

17 Fluorometer Fluorometer analysis of PCR product from convective device with aspect ratio 3

18 Results from PCR performed in the thermocycler.....

19 Contour plots of temperature and velocity in the $\mathrm{XZ}$ and $\mathrm{YZ}$ planes for cylinder with aspect ratio 3

20 Typical streamtrace in cylinder with aspect ratio 3

21 Typical streamtrace in cylinder with aspect ratio 9

22 Temperature distribution of mapping function $f$

23 Schematic of temperature correction used to estimate doubling time

24 Doubling times as a function of Damköhler number ....

25 Variation of the doubling time as a function of Damköhler number

26 Plot of the doubling time vs. Damköhler number on the log scale

27 Poincaré maps for a single streamtrace for cylinders with aspect ratio 9 and 3 respectively.

28 Plots showing the sine function and the random function respectively .....

29 Plots showing the log-log plot of the correlation sum versus the distance $r$ for the cylinders with aspect ratio 3 and 9 respectively

30 Correlation dimensions for cylinders of varying volumes and aspect ratios

31 Temperature contours for $10 \mu \mathrm{L}$ cylinders and different aspect ratios 
FIGURE Page

33 Temperature contours for cylinders of volume $50 \mu \mathrm{L}$ and aspect ratios.... 57

34 Schematic of points used for constant aspect ratios 3 and 8, for constant volume $30 \mathrm{ul}$ and varying Rayleigh numbers..................................... 58

35 Plots of the $\mathrm{z}$ velocity contours, streamtraces and Poincare sections for cylinder with aspect ratio 3 and $\Delta \mathrm{T}=5^{\circ} \mathrm{C}$

36 Plots of the $\mathrm{z}$ velocity contours, streamtraces and Poincaré sections for cylinder with aspect ratio 8 and $\Delta \mathrm{T}=35^{\circ} \mathrm{C}$

37 Schematic of points used for cylinders of constant volume $30 \mathrm{ul}$ and constant Rayleigh number $=10^{7}$ and varying aspect ratios

38 Plots of the $\mathrm{z}$ velocity contours, streamtraces and Poincaré sections for cylinder with aspect ratio 8 and $\Delta \mathrm{T}=27^{\circ} \mathrm{C}$

39 Plots of the $\mathrm{z}$ velocity contours, streamtraces and Poincaré sections for cylinder with aspect ratio 6 and $\Delta \mathrm{T}=47^{\circ} \mathrm{C}$

40 Contour plots of velocity magnitude in the $\mathrm{XZ}$ plane for cylinder with aspect ratio 3 and $\Delta \mathrm{T}=189^{\circ} \mathrm{C}$, taken at different time instants....

41 Contour plots of velocity magnitude in the XZ plane for cylinder with aspect ratio 3 and $\Delta \mathrm{T}=189^{\circ} \mathrm{C}$, taken at different time instants.....

42 Schematic of experimental setup for particle image velocimetry....

43 Cylindrical plexiglas block with an inner cylinder drilled at its center .....

44 Cylindrical plexiglas block with an inner cylinder drilled at its center .....

45 A rectangular mold made up of PDMS, made from a plexiglas rectangular mold.

46 Distribution of laser power over time, showing a Gaussian distribution of the laser fluctuations

47 Typical image obtained from the PIV experiment

48 Contour and vector plots of velocity for the numerical simulation taken at time $\mathrm{t}=40$ seconds 
FIGURE Page

49 Intensity of the light recorded by the spectrophotometer with concentration of Fluorescein $1.6 \mathrm{mg} / \mathrm{L}$, and for Rhodamine $0.8 \mathrm{mg} / \mathrm{L} \ldots \ldots .$.

50 Intensity of the light recorded by the spectrophotometer, with concentration of Fluorescein $20 \mathrm{mg} / \mathrm{L}$, and for Rhodamine $10 \mathrm{mg} / \mathrm{L} \ldots \ldots \ldots .$.

51 Images taken from the LIF experiment, illustrating the negligible photobleaching effect of the laser on the solution.....

52 Sample images from the LIF experiment, taken with the Fluorescein and Rhodamine filters, respectively

53 Temperature contours from the numerical simulations of the LIF experiment in the $\mathrm{YZ}$ and $\mathrm{XZ}$ planes

54 Screenshot of movie with the digital ruler in the background

55 Velocity in the $\mathrm{z}$ direction for two arbitrarily chosen particles from the numerical simulation of visualization experiment using the optical microscope 


\section{LIST OF TABLES}

TABLE Page

1 Typical temperature and time requirements for each step of the polymerase chain reaction ........................................................... 5

2 Comparison of different choices for the characteristic timescale $t^{*}$ on different dimensionless groups appearing in equation $8 \ldots \ldots \ldots \ldots \ldots \ldots \ldots \ldots . . . . . . . . . .36$ 


\section{CHAPTER I}

\section{INTRODUCTION: NATURAL CONVECTION IN BIOCHEMICAL REACTIONS RAYLEIGH-BÉNARD CONVECTION}

A Rayleigh-Bénard convective system is one where a confined layer of fluid is heated from below. Depending on the geometry and fluid properties, a situation can arise where the fluid density at the bottom becomes lower than at the top, thus bringing about a 'top-heavy' unstable arrangement, resulting in lighter fluid moving to the top while the heavy fluid moves to the bottom. Here, buoyancy acts as the destabilizing force and the viscous and thermal diffusion forces act as the restoring forces. The unstable nature of this arrangement is characterized by the Rayleigh number defined in Eq. (1).

$$
R a=\frac{g \beta \Delta T h^{3}}{v \alpha}
$$

The phenomenon of Rayleigh-Bénard convection is shown in Fig. 1. The RayleighBénard convection system has undergone intense experimental and theoretical studies
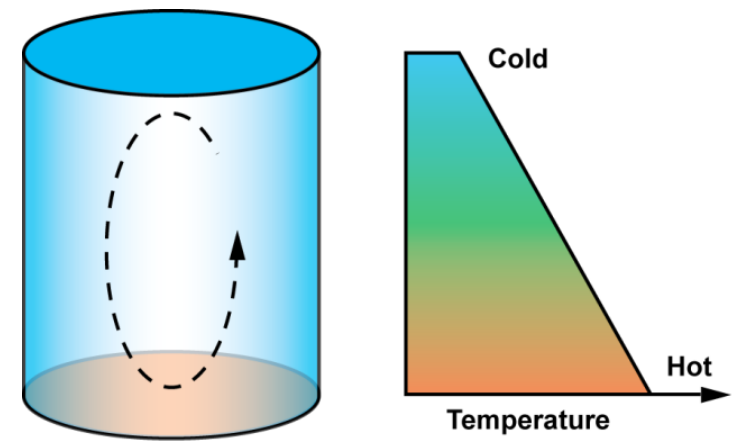

Fig. 1 Schematic of Rayleigh-Bénard convection in a cylinder.

This dissertation follows the style of ASME Journal of Heat Transfer. 
over the course of the past century. A majority of the work has focused on flows generated in low aspect ratio geometries where the effect of the sidewalls is ignored and the fluid is subjected to extremely small temperature gradients which impose negligible variations in fluid properties. Under these conditions, the critical value of Rayleigh number associated with the onset of flow occurs in the vicinity of 1700 [1]. In the case of high aspect ratio geometries, the situation is more complex because the effect of the sidewalls cannot be ignored [2-4]. An analytical stability analysis of convective flow involving insulating sidewall boundary conditions yields an approximate scaling of the order of $R a_{\text {crit }}=1087(\mathrm{~h} / \mathrm{d})^{4}[4]$.

The fluid flow configuration in a cylinder heated from below depends on three critical parameters - the Rayleigh number $(R a)$, Prandtl number $(\operatorname{Pr}=v / \alpha)$ and aspect ratio $(h / d)$. In the case of insulating sidewalls, a number of flow profiles are possible which are discussed in [5] and are highlighted in Fig. 2. The most stable critical mode corresponds to non-axisymmetric motion about the vertical plane of the cavity (i.e. rising in one half of the cavity, falling in the other half, indicated by an arrow in Fig. 2). Thus, it may be seen that for a specific fluid, based on the Rayleigh number and the aspect ratio, a number of flow situations are possible. The phenomenon of natural convection in enclosures has also been used in commercial products like the Lava Lamp where differential heating of two immiscible liquids leads to interesting visual patterns in the flow. In the current work, we present an application of the Rayleigh-Bénard convection system to perform a biochemical reaction. 


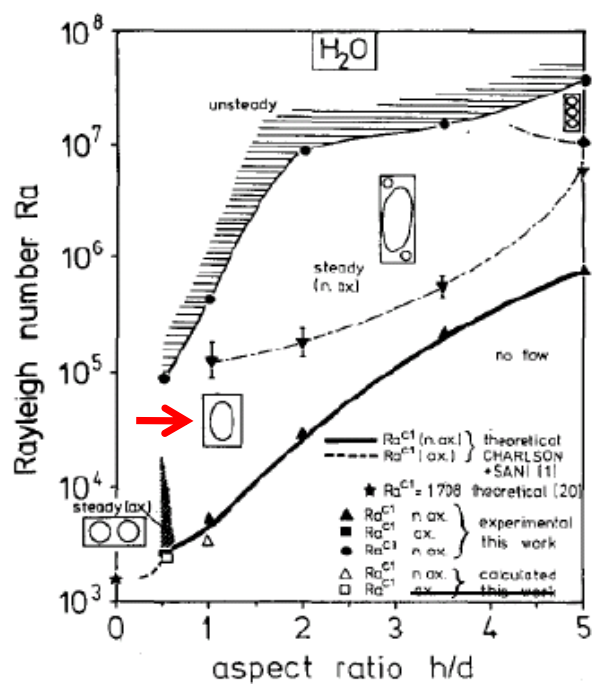

Fig. 2 Stability diagram for $\mathrm{H}_{2} \mathrm{O}(\mathrm{Pr}=6.7)$. Critical Rayleigh numbers for different values of $h / d$ [5]. Most stable critical mode is indicated by an arrow. (Reproduced with permission from Elsevier Limited)

\section{POLYMERASE CHAIN REACTION}

The polymerase chain reaction $(\mathrm{PCR})$ has become an integral tool for the molecular biologist [6]. It was invented by Kary Mullis in 1983 for which he received the Nobel Prize in chemistry in 1993. Since then, the impact of PCR on biological and medical research has been drastic by rapidly speeding the rate of progress of the study of genes and genomes [6]. PCR continues to be an indispensable tool in a diverse array of bioanalysis applications including medical diagnostics, pathogen and infectious disease detection, forensics and population-scale polymorphism and mutation studies [7-13]. The PCR's inherently robust capacity for efficient DNA replication has been significantly extended through development of multiplex assays that allow coamplification of several target sequences so that multiple genomic signatures can be simultaneously detected. Similarly, capabilities to amplify long targets $(>1 \mathrm{~kb})$ have 
proven essential in genome-wide analysis efforts.

A typical polymerase chain reaction involves a reaction volume that contains a buffer solution, a set of primers that are specific to the target DNA strand to be copied, a DNA template that acts as the starter and enzymes that catalyze the reaction. The DNA template is initially double stranded, consisting of two single strands held together by hydrogen bonds. At the denaturing temperature, it separates into two single strands. This is followed by an annealing process at a lower temperature where the primers bind to specific regions of the single stranded DNA. Finally, the primers extend at an intermediate temperature in the presence of the enzyme, thus creating a double stranded molecule from the single stranded DNA. This double stranded DNA once again denatures forming two single stranded DNA templates at the denaturing temperature, and this process is repeated for a specific number of cycles. This process is represented in Fig. 3. Each of the processes of denaturing, annealing and extension takes place at different temperatures for different amounts of time - these conditions are specific to the nature of reagents being used in the reaction. The range of temperatures typically used is shown in Table (1).

Despite the complexity of the individual steps that occur in PCR, the overall reaction may be easily performed by exposing the reagents to the correct temperatures for a required number of cycles (usually about 30-40), which then leads to an exponential increase in the concentration of the required template. Thus, it is possible to start from a very small amount of template and then produce a very high concentration of the target region by using PCR. The temperature cycling protocol for a PCR is 


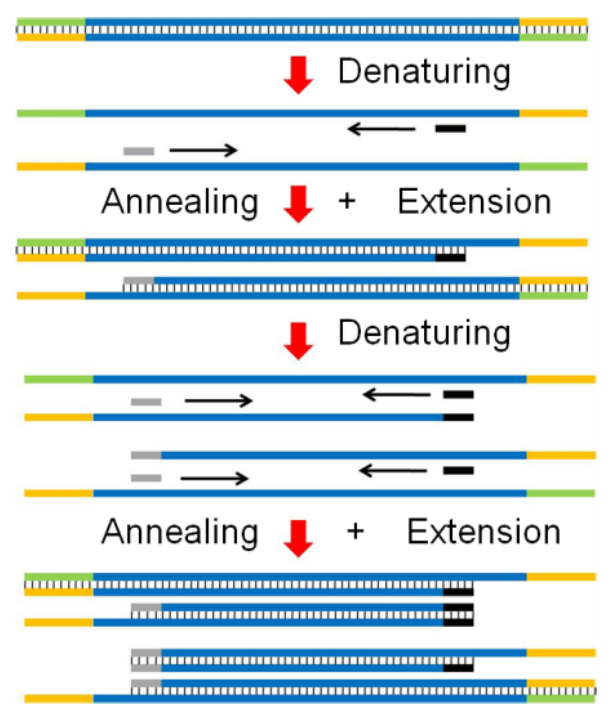

Fig. 3 Schematic of the process of polymerase chain reaction showing the various steps involved in a cycle.

Table 1. Typical temperature and time requirements for each step of the polymerase chain reaction $[14]$.

\begin{tabular}{|c|c|c|c|}
\hline Step & Temperature $\left({ }^{\circ} \mathrm{C}\right)$ & Time (s) & Reaction time \\
\hline Denaturation & $90-96$ & $30-60$ & $\sim$ Instantaneous \\
\hline Annealing & $55-60$ & 30 & $\sim$ Instantaneous \\
\hline Extension & $72-75$ & 30 & $\sim 70$ bases $/ \mathrm{s}$ \\
\hline
\end{tabular}

typically entered into a benchtop instrument called a thermocycler (T1 Thermocycler $\AA$, Biometra, shown in Fig. 4). However, this process takes up to 3 hrs. per reaction. Surprisingly, these limitations have little to do with the reaction kinetics but are instead a reflection of the inefficient design of the instruments used to perform the thermocycling. These instruments essentially consist of metal heating blocks whose temperature is 
regulated by computer-controlled thermoelectric heaters. This design's inherently high heat capacity severely limits the attainable heating and cooling rates and consumes considerable electrical power. These problems are magnified by the low thermal conductivity plastics (e.g., polypropylene) used to construct the reaction tubes and multiwell plates, resulting in the need to hold the temperature constant at each stage of the reaction for a significant period of time so that the entire reagent volume can equilibrate.

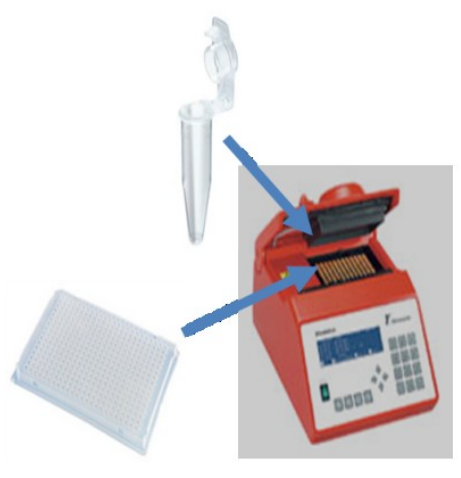

T1 Thermocycler ${ }^{\bullet}$ (Biometra)

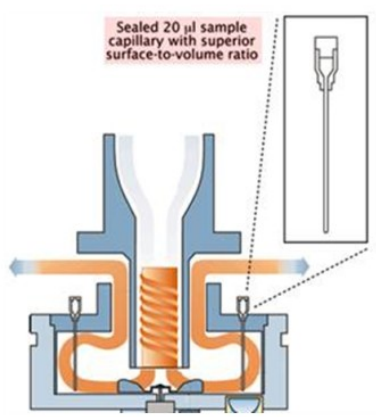

LightCycler (Roche)

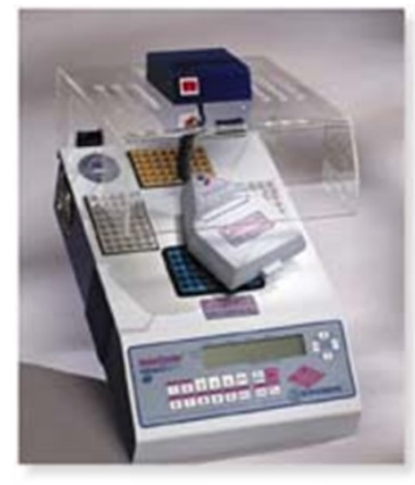

Robocycler ${ }^{\bullet}$ (Stratagene)

Fig. 4 Commercial bench top PCR instrumentation.

Consequently, the majority of time and electrical power consumed in the thermocycling process is wasted regulating the temperature of elements associated with the instrument hardware that are not involved in the reaction. Other commercial devices include an apparatus containing a very thin glass capillary which contains the PCR reagent mixture (Lightcycler ${ }^{\circledR}$, Roche, shown in Fig. 4). Here, the capillary is heated and cooled to the correct PCR temperatures using air driven by forced convection as the 
heating mechanism. Due to the enhanced area to volume ratio, it is expected that the reaction can proceed very quickly compared to a multiwell thermocycler. However, this provides difficulties in loading the reagents due to the extremely tiny diameter of the capillary. Another commercial bench top instrument is the Robocycler ${ }^{\circledR}$, Stratagene, shown in Fig. 4 - here, the apparatus consists of three metallic heating blocks, each maintained at the correct PCR temperature (i.e. denaturing, annealing and extension temperatures) and a robotic arm moves the vial containing the PCR reagents from one block to another and holds it at the correct temperature for the desired time, which makes the device cumbersome to use.

\section{NATURAL CONVECTION BASED POLYMERASE CHAIN REACTION}

Recently, natural convection has been explored as a novel method of performing PCR. Here, Rayleigh-Bénard convection was harnessed to transport reactants through different temperature zones associated with the reaction, thus eliminating the need for repeated heating and cooling that must be implemented in a conventional instrument $[15,16]$. The main advantage of this system was that the temperature control was simple - the only heating required was to maintain the temperatures of the top and bottom surfaces of a cylinder at a constant value. Other advantages include inexpensive hardware, faster reaction speeds and easy integration with existing protocols and liquid handling systems. Typically, reaction volumes for PCR are of the order of tens of microliters. In order to consider a cylindrical system where natural convection would aid PCR, two possibilities were analysed for the design of the reaction chamber, one where the aspect ratio is less than 1 and the other with aspect ratio greater than 1 . The problem 
with a cylinder whose aspect ratio is less than 1 is that for the reaction volumes under consideration and the critical Rayleigh number that must be achieved in order for natural convection to take place, the dimensions of the system are very small and make it difficult to maintain a temperature gradient across the fluid layer. Another problem was that the fluid will recirculate too fast for the reaction to occur optimally. Therefore, the second possibility was explored where the aspect ratio of the cylinder is greater than 1 . In this case, it was easier to establish a temperature gradient across the height of the cylinder.

The previous idea explored was that establishing a temperature gradient over a cylinder with an aspect ratio within a range of about 8-10 will establish a single steady convective cell which will aid in the reaction. This idea was investigated in $[15,16]$ and the main results are highlighted here. A prototype multiwell convective thermocycler device was constructed using two aluminum plates: a bottom plate heated with inexpensive cartridge heaters using a microprocesser driven temperature controller to regulate thermal output and an upper plate whose temperature is regulated with a recirculating water bath, as shown in Fig. 5. This design offers a substantial level of flexibility through the use of interchangable plastic PCR reaction chamber "cartridges" that incorporate an array of cylindrical reactors. The reagents used were from a commercial PCR kit that amplified a 191 base pair (bp) fragment associated with membrane channel proteins M1 and M2 of the influenza-A virus (Maxim Biosciences) together with the AmpliTaq enzyme (Applied Biosystems). The convective PCR system was set with the temperature of the top plate at $61{ }^{\circ} \mathrm{C}$ and that of the bottom plate at 96 
${ }^{\circ} \mathrm{C}$ and the reaction was performed for $40 \mathrm{~min}$. A control reaction was also performed in

a commercial thermocycler (T-Gradient, Biometra) with a three temperature protocol $\left(61-72-95{ }^{\circ} \mathrm{C}, 1 \mathrm{~min}\right.$ each, 35 cycles) for a total time of $2.5 \mathrm{hrs}$.

(a)

(b)
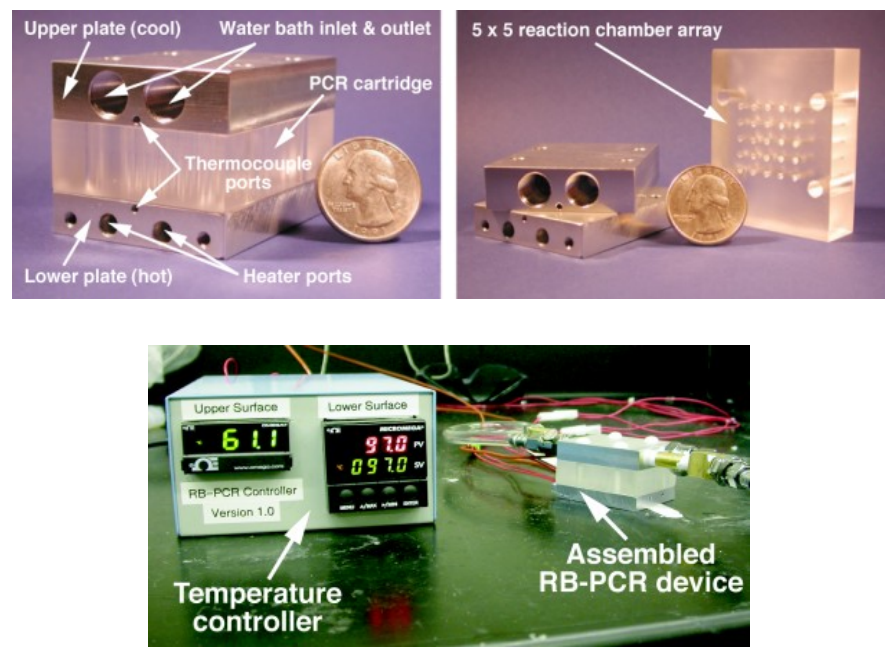

Fig. 5(a) Prototype of cavity-based convective PCR thermocycling device incorporating a Plexiglas PCR cartridge sandwiched between a top plate connected to a circulating water bath and a bottom plate incorporating cartridge heaters. (b) Assembled convective thermocycler with temperature control unit.

The product samples from the reaction were analyzed by gel electrophoresis. A multi-well containing gel was prepared using $2 \%$ agarose mixture and submerged into a buffer solution. DNA samples were mixed with a flourescent dye, and loaded into the wells and an electric potential was applied. The principle involved in this analysis is that DNA molecules are negatively charged and therefore, in the presence of an electric field, migrate toward the positive electrode with a size dependent speed (smaller fragements move faster). The flouroscent dye aids in visual analysis of the gel in the presence of 
ultraviolet light and a photoimage of the gel can be used for analysis. In order to characterize the length of the target DNA strand, a DNA ladder is used for comparison. For the current case where the target DNA strand has a length of $191 \mathrm{bp}$, the DNA ladder consists of markers from 100-1000 base pairs. A schematic of this procedure is shown in Fig. 6.

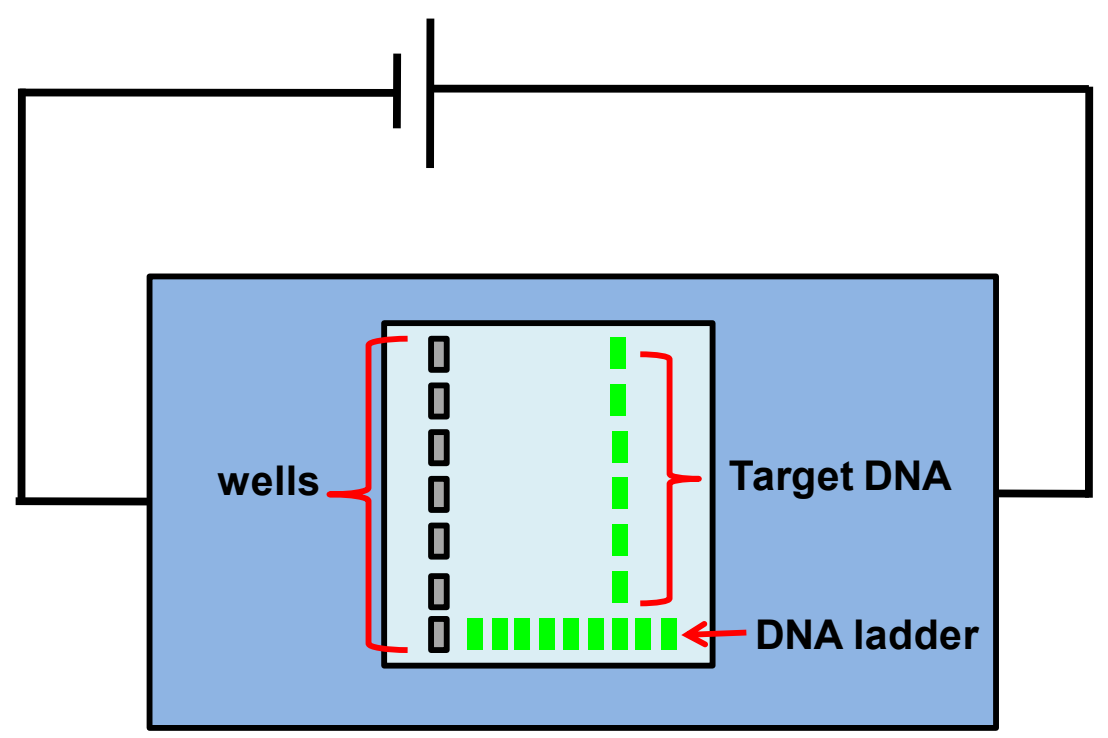

Fig. 6 Schematic of the process of gel electrophoresis

The result of the analysis is shown in Fig. 7. It can be seen that the convective PCR system gave the same product as the conventional system in a shorter period of time. This confirmed that it is possible to run PCR in a Rayleigh-Bénard system accurately. Reactions were also analyzed for different run times in the convective PCR system and it was observed that at least $30 \mathrm{~min}$. of reaction time were needed before a substantial amount of product was visible. These results are shown in Fig. 8. This phenomenon (i.e. the initial "dead time" before reaction product was evident) needed 


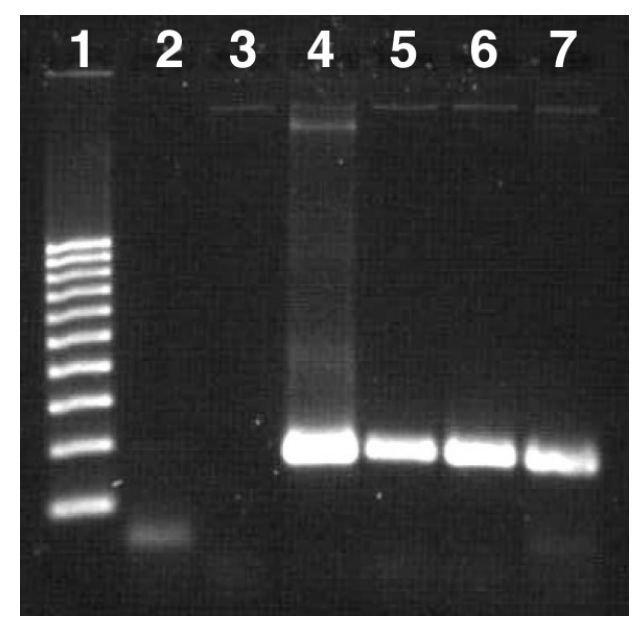

Fig. 7 Comparison of products obtained Fig. 8 Comparison of reaction times in the from a RB-PCR system with conventional RB-PCR device. Lane 1: $100 \mathrm{bp}$ ladder, thermocycler (all reactions run for 40 lane 2: Reaction time $=30 \mathrm{~min}$. lane 3 : min.). Lane 1: 100 bp ladder, lane 2: Reaction time $=35 \mathrm{~min}$. lane 4: Reaction negative control with no template, lane $3:$ time $=40 \mathrm{~min}$. lane 5: Reaction time $=45$ negative control with no enzyme, lane 4: $\min$. lane 6: Reaction time $=50 \mathrm{~min}$. thermocycler using a 3-temperature cycling protocol (anneal: $61{ }^{\circ} \mathrm{C}$, extend: 72 ${ }^{\circ} \mathrm{C}$, denature: $95{ }^{\circ} \mathrm{C}, 35$ cycles), lanes 5-7: PCR product from 3 parallel reactions in the multiwell device.

to be investigated further. But in order to understand the effect of run times on the reaction, it is necessary to consider the dynamics of fluid flow inside a Rayleigh-Bénard system in more detail. This would then help to optimize the design of the reaction chamber and ultimately reduce the reaction time. This is the focus of the current work.

\section{OTHER CONVECTIVE DEVICES IN LITERATURE}

Braun et al. [17] performed convective PCR using a micro immersion heater. The cylindrical chamber consisted of a $1.75 \mathrm{~mm}$ thick silicone rubber sheet with a $4 \mathrm{~mm}$ hole. 
A glass sheet was used at the bottom of the hole to seal the fluid and the fluid was exposed to air at the top. They simulated their setup using FEMLAB simulations, where they modeled the top surface as being adiabatic and the sidewalls were modeled with the temperature maintained at a constant value of $55^{\circ} \mathrm{C}$. They were able to amplify a $96 \mathrm{bp}$ DNA template in 20 min using their setup. Braun et al [18] also performed convective PCR using an infra-red beam as the heating source. The setup consisted of a cylindrical chamber $1 \mathrm{~mm}$ in height and $5 \mathrm{~mm}$ in diameter. The center of the cylinder was heated using an infra-red beam and the sidewalls were maintained at $52{ }^{\circ} \mathrm{C}$. They were able to amplify $96 \mathrm{bp}$ DNA template in $25 \mathrm{~min}$. They also performed two dimensional simulations of their setup in FEMLAB. Chung et al [19] created a palmtop PCR chip using three heaters in a triangular shape. A channel through which the PCR reagents flow, was created along the sides of the heater, its cross-section being $1 \mathrm{~mm} \times 1 \mathrm{~mm}$. The duration of the PCR cycle can be changed by inclining the device with respect to gravity. They were able to amplify a previously prepared $127 \mathrm{bp}$ DNA template in 10 min, whereas when they used a 470 bp genomic DNA as the starting template, the reaction occurred in 20 min. Wheeler et al [20] used two separate circuit boards, assembled in one package, to perform PCR using natural convection. Layers of photolithographically patterned polymer films form an elliptic channel on each circuit board. A specifically designed $12 \mu \mathrm{m}$ thick polypropylene bag is loaded with the sample and sandwiched between the two circuit boards. Surface mounted resistors and resistance temperature detectors are used as heaters and temperature sensors. They were able to amplify $90 \mathrm{bp}$ target DNA in 30 min using this setup. Chen et al [21] used a loop based reactor with 
three heaters to perform PCR using natural convection. In their setup, they were able to change the angle of inclination of the loop to adjust the flow rate of the reagents within. They were able to amplify 700 and 350 bp genomic DNA in $73 \mathrm{~min}$. They also performed three dimensional simulations of the flow inside the loop based reactor using CFD-ACE+.

Ugaz et al [22] used a triangular loop based reactor with two heaters, to perform PCR using natural convection. the loop was created using FEP tubing, which are biocompatible and are optically transparent. Furthermore, they do not require additional surface treatment techniques to load the reagents. The two heaters were set to maintain temperatures of $94{ }^{\circ} \mathrm{C}$ (denaturing) and $72{ }^{\circ} \mathrm{C}$ (extension), so that the third side passively attains a temperature of $60{ }^{\circ} \mathrm{C}$ (annealing). They were able to amplify $1190 \mathrm{bp}$ DNA template in 50 min, using this method. Ugaz et al [23] also created a pocket sized convective thermocycler which could be powered by 2 AA batteries. The setup consisted of three aluminum blocks with thermal interconnections so that they can regulate the heat flow between the different blocks. One block is heated to the denaturing temperature $\left(95^{\circ} \mathrm{C}\right)$ and the system is so designed that the other blocks passively reach the correct temperatures for the annealing and extension reactions. Using this setup, they were able to amplify 1300 bp DNA in 50 min. Ugaz et al [16] also created a rectangular loop based PCR device with two heaters at the top and bottom. They were able to amplify 297 bp DNA in $30 \mathrm{~min}$.

Yao et al [24] created a convection cavity using PDMS (polydimethylsiloxane), with two microheaters at the top and bottom surfaces. They amplified a 406 bp DNA 
template in 6-14 min using a range of aspect ratios between 1 and 7 . They also performed numerical simulations using CFD-RC. Zhang et al [25] created a fin based design, where they used a combination of copper and aluminum fins as the heat sources for a tubing containing the PCR reagents which was wrapped around the fins. They were able to amplify 112 bp DNA in 45 min using this method.

In the current work, we seek to perform computational fluid dynamics simulations of Rayleigh-Bénard convection for PCR conditions. Using these simulations, we would like to understand the effect of the Rayleigh number and aspect ratio on the flow inside a cylinder. Based on the results of the numerical simulations, experiments will also be performed using optimized reactor geometries to verify if the rate of reaction is indeed optimized. The effect of fluid flow on the rate of the polymerase chain reaction will also be studied by performing numerical simulations coupling the flow to the reaction kinetics, which will give us further insight into the interplay between flow and reaction. The nature of the fluid flow will also be studied using Poincaré maps and correlation dimensions which will provide qualitative and quantitative measures of chaos in a given flow respectively. Finally, flow measurement techniques like particle image velocimetry and laser induced fluorescence will be used to evaluate velocity and temperature fields inside the cylinder to provide validation of numerical simulations. 


\section{CHAPTER II}

\section{COMPUTATIONAL FLUID DYNAMICS SIMULATIONS}

The goal of the current work is to model the flow systems using computational fluid dynamics. But, before going any further, it is necessary to establish the fact that using water as the working fluid will be sufficient to predict the flow field in a cylinder containing biochemical reagents. The PCR reagent mixture is an aqueous biomolecule solution at a concentration so dilute that the fluid properties are only minimally different from those of pure water. Moreover, these properties remain essentially constant during the course of the reaction even though the concentration continually increases. To determine whether the continually increasing concentration of target DNA fragments during PCR can significantly influence the bulk fluid properties, we use the Einstein model for viscosity of a dilute suspension of spheres given in Eq. (2).

$$
\eta=\eta_{o}(1+2.5 \phi)
$$

The radius of gyration $(R g)$ of the DNA molecule estimated using the KratkyParod equation for semiflexible polymers [26] allows one to calculate the size of an equivalent sphere representing the DNA coil. Consider a reaction where 1 billion copies of a 500 base-long double-stranded DNA target are replicated from each of 1,000 template DNA molecules initially present in a $10 \mu \mathrm{L}$ reactor. Applying characteristic values (persistence length $\sim 50 \mathrm{~nm}$, contour length per base $\sim 0.4 \mathrm{~nm}$ ) yields $R g=42 \mathrm{~nm}$. This yields a corresponding volume fraction of replicated DNA in the $10 \mu \mathrm{L}$ reactor of $\varnothing$ $=3.1 \%$, and a corresponding viscosity increase of only $\sim 7 \%$ above that of pure water (this is also a very conservative estimate because it assumes $100 \%$ replication efficiency 
during 30 cycles of PCR). Thus, we conclude that the fluid properties exhibit only minimal deviations from those of pure water under the conditions of interest to in this work. Finally, any potential viscoelastic effects associated with the DNA present in the PCR reaction mixture have been shown to exert a negligible influence on the stability of Rayleigh-Bénard convection owing to the relatively weak nature of the convective flow field under PCR conditions [27].

In order to optimize the reaction chamber so that the reactants travel through the different temperature zones for sufficient amount of time, it is necessary to perform a numerical study which would take into account the parameters of the system and predict flow patterns in the cylinder. In the current work, we considered reaction volumes ranging from $0.005 \mu \mathrm{L}$ to $50 \mu \mathrm{L}$ and a range of aspect ratios from 0.5 to 10 . For each of these cases, it is possible to compute the dimensions of the cylinder and the Rayleigh number of the system, given that the temperature difference between the bottom and top surface is $35 \mathrm{~K}$. The resulting range of Rayleigh numbers for each of these cases is given in Fig. 9. It can be seen that there exist a range of reaction volumes $(0.005 \mu \mathrm{L}-1 \mu \mathrm{L})$ that would yield a no-flow situation in the cylinder, i.e. the dominant mode of heat transfer is pure conduction. Therefore, these cases are not useful for the current work. The reaction volumes that are of interest to us are in the range of $10-50 \mu \mathrm{L}$ and these cases have Rayleigh numbers varying from $5 \times 10^{3}$ to $5 \times 10^{7}$. Without loss of generality, we considered a typical reaction volume of $30 \mu \mathrm{L}$ in a cylinder with aspect ratios ranging from 0.5 to 10 . A cylindrical geometry was created for each of these cases with the appropriate dimensions using GAMBIT and this model was then meshed with 


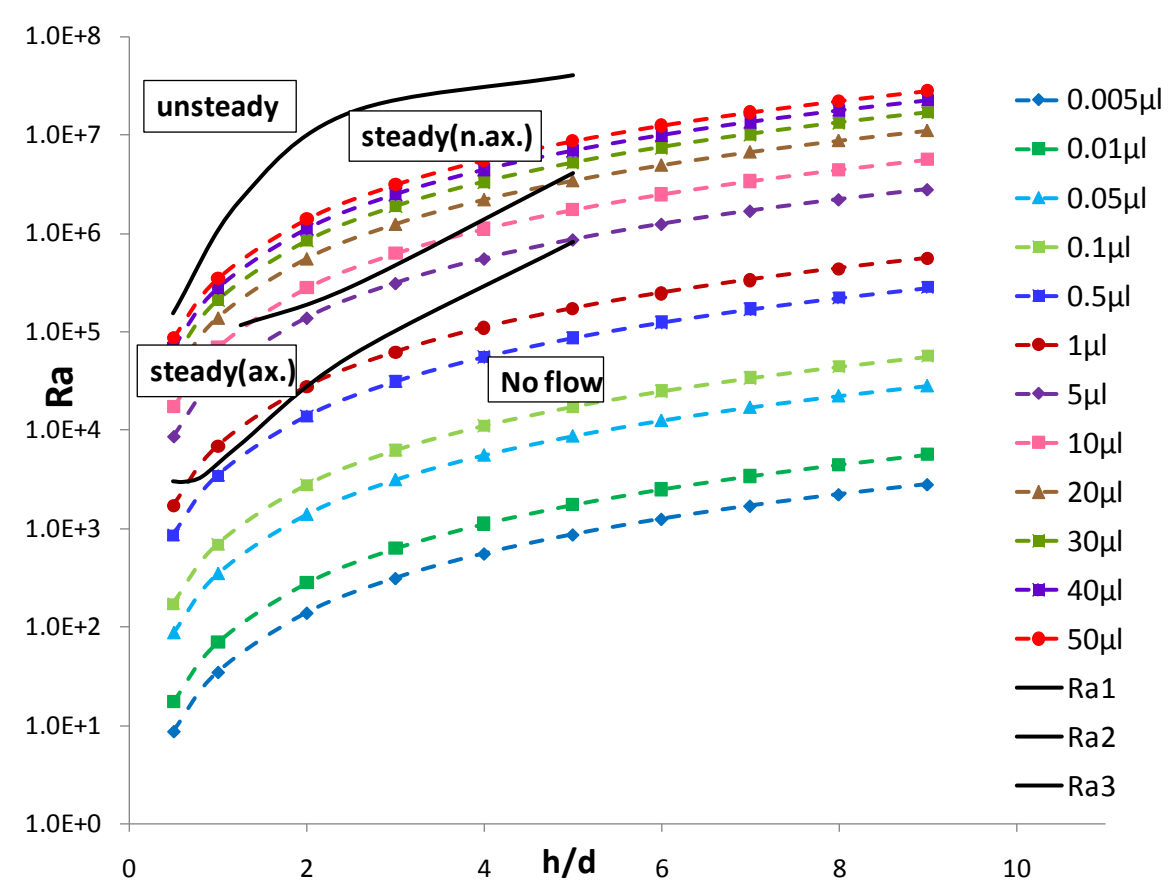

Fig. 9 Spectrum of Rayleigh numbers vs. aspect ratio (h/d) for different reaction volumes. $\mathrm{Ra}_{1}, \mathrm{Ra}_{2}$ and $\mathrm{Ra}_{3}$ refer to the results obtained by Müller[5].

hexahedral elements. This geometry was then solved using FLUENT (ver. 6.0.12) with a three dimensional, laminar and steady-state set of equations. The working fluid was defined as water, with Boussinesq properties evaluated at $95{ }^{\circ} \mathrm{C}$. The top surface of the cylinder was maintained at $62{ }^{\circ} \mathrm{C}$ and the bottom surface was maintained at $97{ }^{\circ} \mathrm{C}$. The sidewalls of the cylinder were considered to be insulated. Gravity was included as a body force and a converged solution was obtained by using second-order upwind discretization schemes for momentum and energy, PRESTO scheme for pressure and SIMPLE scheme for pressure-velocity coupling. The equations for conservation of mass, momentum and energy written in dimensionless forms are given in Eq. (3-5). 


$$
\begin{gathered}
\vec{\nabla} \cdot \vec{V}=0 \\
\vec{V} \cdot \vec{\nabla} V=-\nabla p+\operatorname{Pr} \nabla^{2} \vec{V}+\operatorname{Ra} \operatorname{Pr} T \overrightarrow{e_{z}} \\
\vec{V} \cdot \vec{\nabla} T=\nabla^{2} T
\end{gathered}
$$

The length is non-dimensionalized with respect to the height of the cylinder $h$, velocity with $\alpha / h$, pressure with $\rho \alpha^{2} / h^{2}$, time with $h^{2} / \alpha$ and temperature $T$ as $\left(T-T_{a}\right) /\left(T_{b}-T_{a}\right)$, where $T_{a}<T_{b}$. The fluid is modeled as having Boussinesq properties:

$$
\beta=\frac{1}{\rho}\left|\frac{\partial \rho}{\partial t}\right|
$$

\section{GRID CONVERGENCE}

A grid convergence study was done by considering the case with aspect ratio 3 . The number of hexahedral cells in each case varied from 7488 to 201058 and the solutions for each case were analysed. By computing the magnitudes of velocity across a

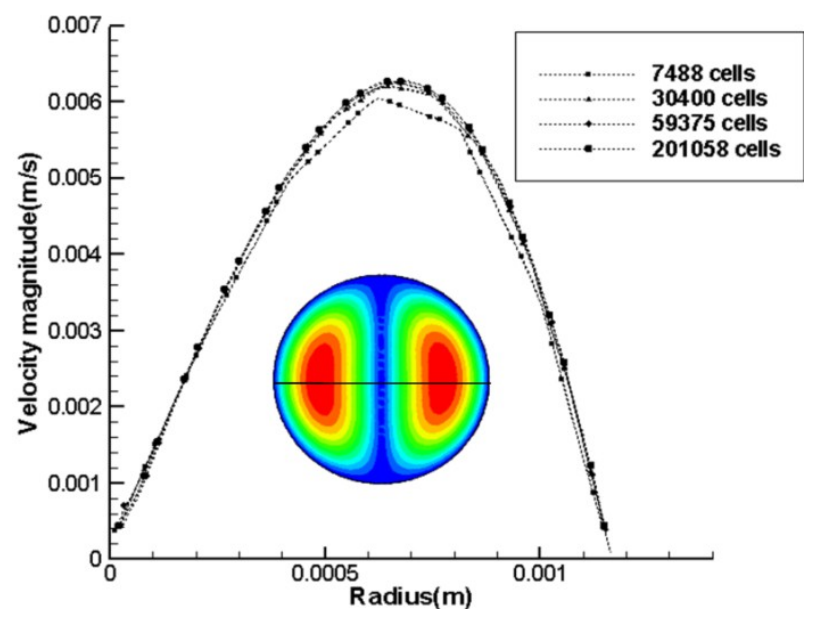

Fig. 10 Grid convergence study showing the magnitudes of velocity along a line drawn at a circular section which bisects the cylinder into two equal parts. 
line drawn along the central section of the cylinder, it has been observed that by increasing the number of cells from 59375 to 201058, the deviation between the solutions is less $1 \%$. These results are shown in Fig. 10. Therefore, in order to attain a balance between computational accuracy and expense, a geometry containing 59375 cells with grid sizes of $\Delta \mathrm{x}=5.188 \times 10^{-5} \mathrm{~m}, \Delta \mathrm{y}=5.188 \times 10^{-5} \mathrm{~m}$ and $\Delta \mathrm{z}=2.335 \times 10^{-5} \mathrm{~m}$ was chosen.

\section{NUMERICAL RESULTS OF SIMULATIONS}

Here, the numerical results of two interesting cases of natural convection in a cylinder are presented, highlighting two distinct flow configurations - one has an aspect ratio of $3\left(h=7.01 \mathrm{~mm}, d=2.34 \mathrm{~mm}, R a=1.85 \times 10^{6}\right)$ and the other has an aspect ratio of 8 ( $\left.h=13.47 \mathrm{~mm}, d=1.68 \mathrm{~mm}, R a=1.32 \times 10^{7}\right)$. First, contour plots of temperature and velocity for each of these cases are presented in Fig. 11. It can be seen from the contours of velocity that a convective flow is generated in both the cases, the maximum velocity being about $6 \mathrm{~mm} / \mathrm{s}(\operatorname{Re} \sim 40)$ for the case with $h / d=3$ and about $3.5 \mathrm{~mm} / \mathrm{s}(\operatorname{Re} \sim 22)$ for the case with $h / d=8$, thus the flow is laminar (Reynolds number was calcuated using the diameter of the cylinder). Now, a closer look at the flow field in obtained by presenting the vector plots of velocity for the two cases, shown in Fig. 12. For the case with aspect ratio 8 , it can be seen that the fluid flow is essentially two dimensional - the fluid at the bottom becomes lighter and rises to the top, while the fluid at the top becomes heavier and sinks to the bottom, creating a stable circulatory flow pattern. However, the case with aspect ratio 3 shows a much different behaviour. As the lighter fluid at the bottom 
(a)h/d=3

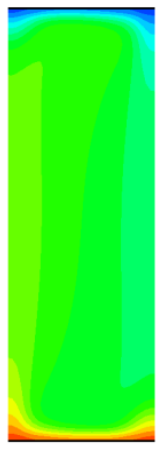

(e) $\mathrm{h} / \mathrm{d}=8$

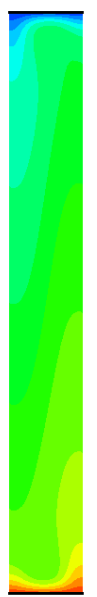

(i) $\mathrm{h} / \mathrm{d}=3$

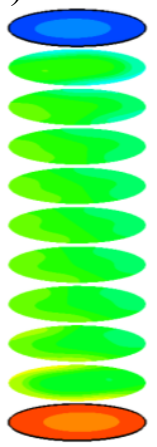

(b) $\mathrm{h} / \mathrm{d}=3$

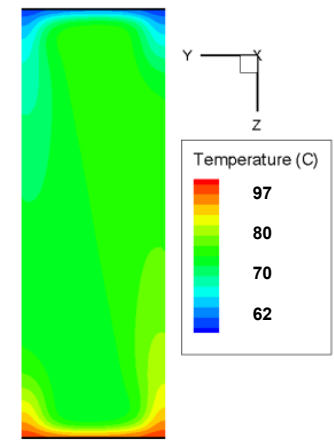

(f) $\mathrm{h} / \mathrm{d}=8$

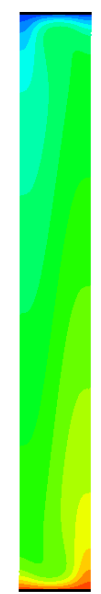

(j) $h / d=3$

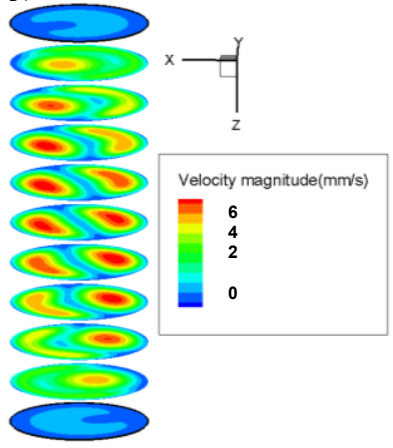

(c) $\mathrm{h} / \mathrm{d}=3$

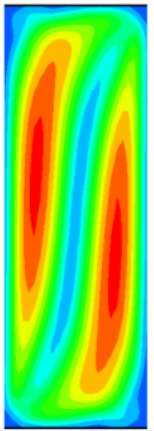

(g) $h / d=8$

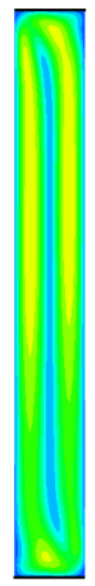

(k) $\mathrm{h} / \mathrm{d}=8$

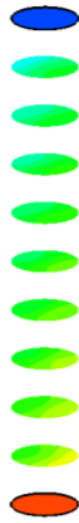

(d) $\mathrm{h} / \mathrm{d}=3$

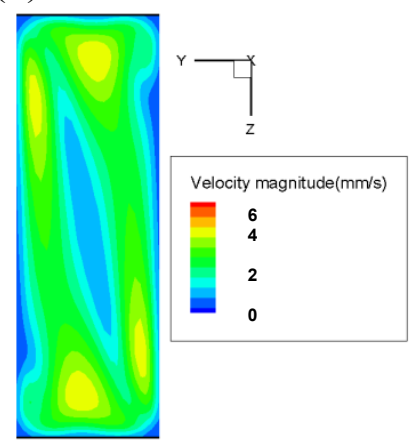

(h) $\mathrm{h} / \mathrm{d}=8$

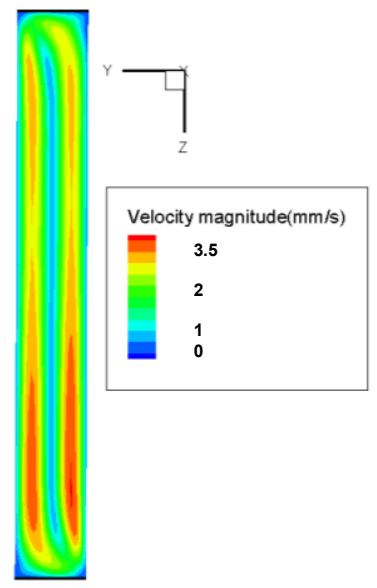

(1) $h / d=8$

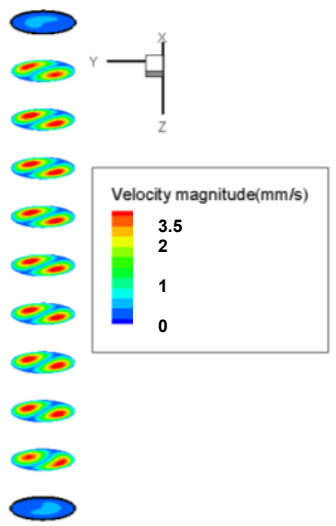

Fig. 11(a)-11(d) Contour plots of temperature and velocity in the XZ and YZ planes for cylinder with aspect ratio 3. Fig. 11(e)-11(h) Contour plots of temperature and velocity in the $\mathrm{XZ}$ and $\mathrm{YZ}$ planes for cylinder with aspect ratio 8 . Fig. 11(i)-11(1) Contour plots of temperature and velocity in the XY planes for cylinder with aspect ratio 3 and 8 , respectively. 
(a) $\mathrm{h} / \mathrm{d}=8$

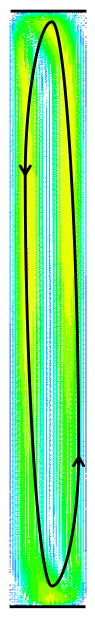

(b) $h / d=8$

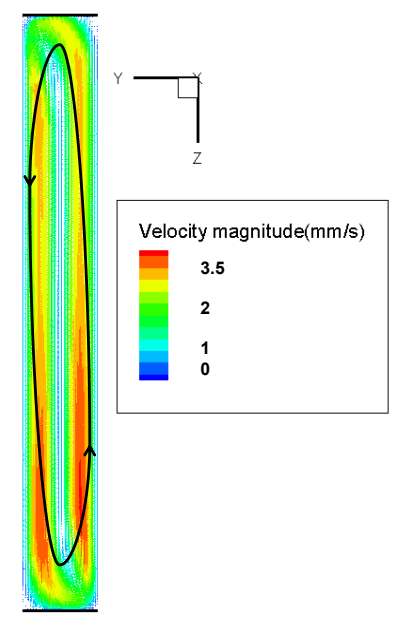

(c) $h / d=3$

(d) $h / d=3$

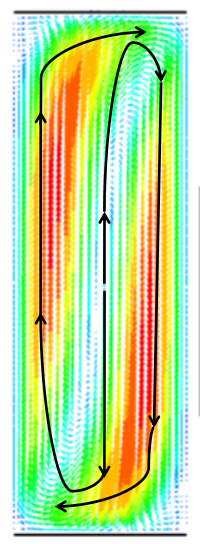

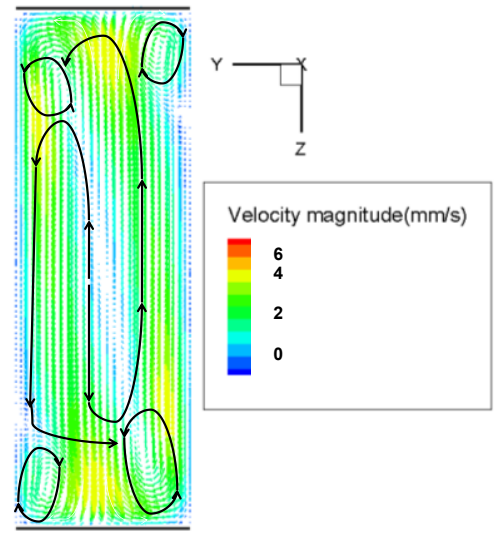

Fig. 12(a)-12(d) Vector plots of velocity in the $\mathrm{XZ}$ and $\mathrm{YZ}$ planes for cylinders with aspect ratio 8 and 3 , respectively.

rises to the top, it circulates around the center of the cylinder, while the heavier fluid at the top circulates around the center of the cylinder as it sinks to the bottom. This is a critical difference in the fluid flow configurations for the two cases. In addition, recirculation zones are present at the upper and lower corners of the cylinder with aspect ratio 3 .

This unusual behaviour prompted further investigation. For this purpose, streamtraces were drawn originating at several locations along a plane bisecting the cylinder in half along its height for both cases. A schematic of the points chosen is shown in Fig. 13. The position, velocity and temperature history of a particle traveling along the streamtraces originating at each of these points was extracted. The pathlines corresponding to particles on streamtraces and the time evolution of their temperatures 


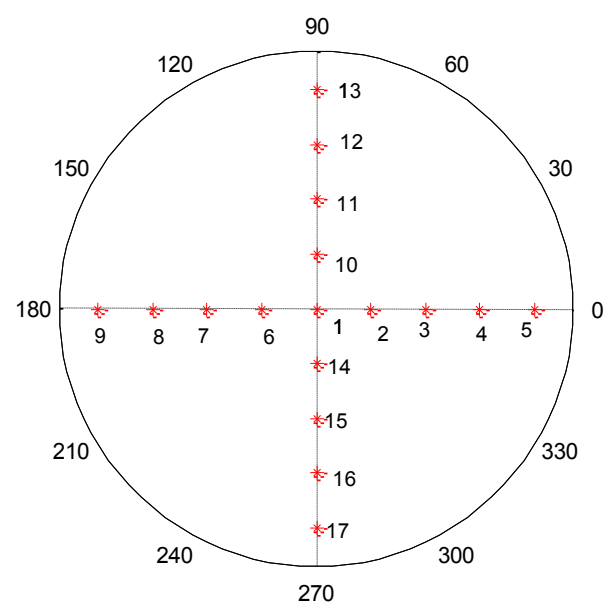

Fig. 13 Schematic of points chosen as starting points for extracting streamtraces.

were plotted, shown in Fig 14. A key observation from this analysis is that the characteristics of the convective flow field can dramatically change within this $R a$ regime. At $h / d=8$, close to the conditions in the reactor designs employed in the previous work $[15,16]$, the fluid element trajectories are essentially closed loops (Fig. 14(a)-14(c)). That is, fluid elements are locked-in to follow the same loop path for a long period of time. Consequently, there is little opportunity for exchange between flow trajectories that expose reagents to the optimal sequence of thermal conditions and other trajectories that do not contribute to amplification. At $h / d=3$, on the other hand, the flow becomes more disordered in the sense that the fluid element trajectories no longer follow closed paths. Thus, fluid elements cover a wide range of trajectories throughout the reactor volume permitting ample opportunity for exchange within the temperature field (Fig. 14(d)-14(f)). 
The CFD analysis also makes it possible to extract the temperature history experienced by a fluid element as it travels through the reactor (Fig. 14(g)-14(h)). In the case of $h / d=8$, the temperature profile is characterized by a periodic oscillation between the upper and lower temperature extremes, mimicking a 2-temperature profile employed

(a) $h / d=8$

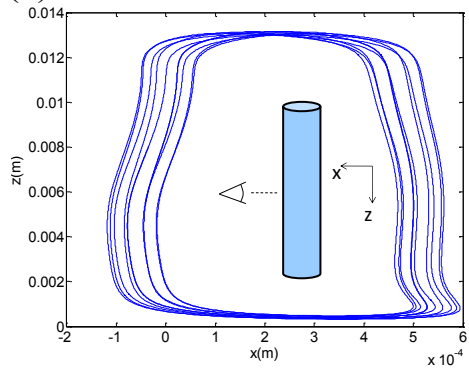

(d) $h / d=3$

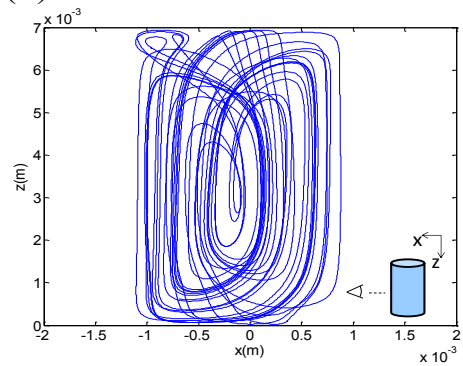

(g) $\mathrm{h} / \mathrm{d}=8$

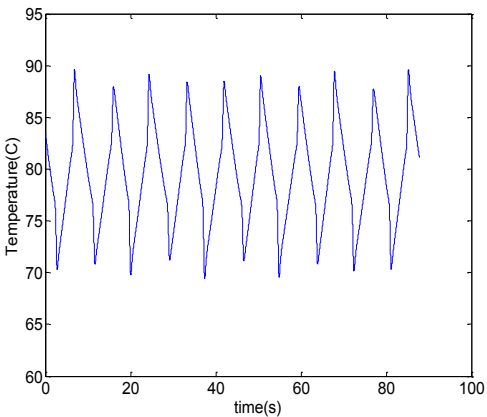

(b) $\mathrm{h} / \mathrm{d}=8$

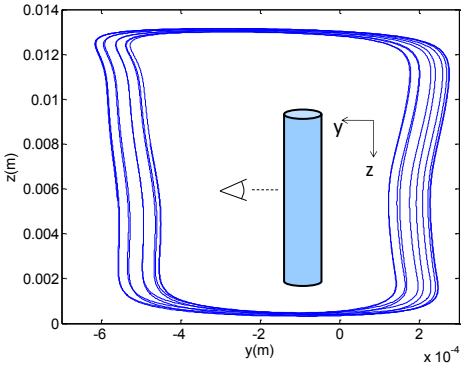

(e) $h / d=3$

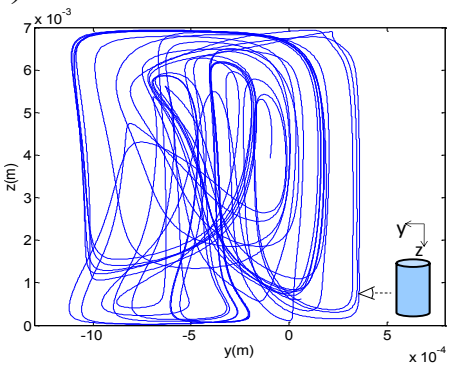

(c) $\mathrm{h} / \mathrm{d}=8$

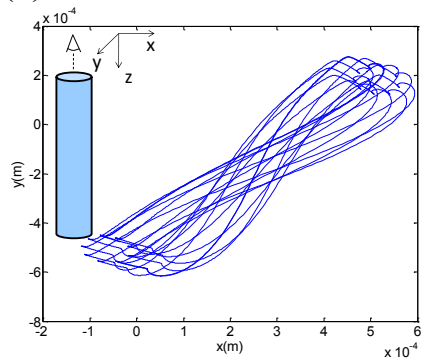

(f) $h / d=3$

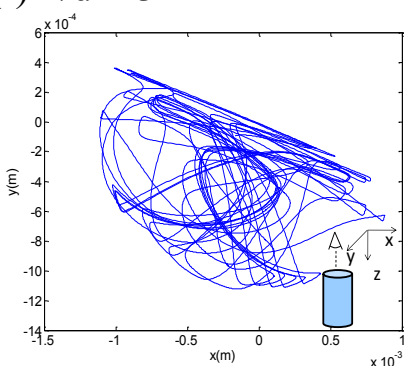

(h) $\mathrm{h} / \mathrm{d}=3$

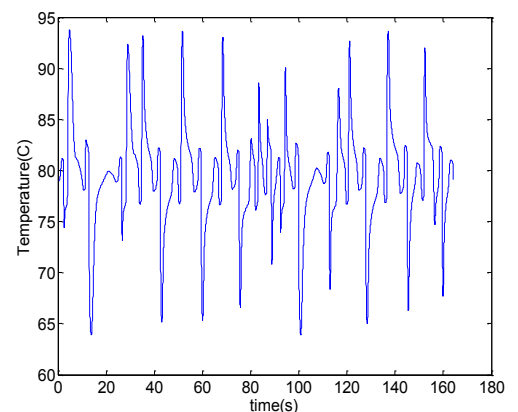

Fig. 14(a) - 14(c) Particle paths in the XZ, YZ and XY planes for cylinders with aspect ratio 8, respectively. Fig. 14(d) - 14(f) Particle paths in the XZ, YZ and XY planes for cylinders with aspect ratio 3 , respectively. Fig. 14(g) - 14(h) Plot of temperature vs. time for a typical particle path in a cylinder with aspect ratio 8 and 3 , respectively. 
in conventional thermocyclers. But the profile is much different at $h / d=3$, displaying increased time at the intermediate temperature corresponding to extension conditions as a consequence of frequent exchange between flow trajectories. Based on this analysis, it was hypothesized that a reactor design corresponding to $h / d=3$ may be more favorable for PCR due to a synergistic combination of (1) continuous exchange among flow paths that would enable reagents an enhanced opportunity to sample the full range of optimal temperature profiles and (2) increased time spent within the extension temperature zone - the rate limiting step of PCR. This hypothesis runs entirely counter to our previous expectation that the stable circulatory flow conditions at $h / d=8$ would be optimal for PCR.

\section{TWO DIMENSIONAL SIMULATIONS OF PCR GEOMETRIES}

In order to understand the need for performing three dimensional simulations as opposed to using results from from two-dimensional simulations of the Rayleigh-Bénard system, we performed additional two dimensional simulations for the cylinder with aspect ratio 3 and volume $30 \mu \mathrm{L}$. The contour and vector plots of velocity for this case are shown in Fig. 15. It can be noticed that the fluid flow is essentially two-dimensional in nature - the fluid circulates between the top and bottom surfaces in a manner similar to the three dimensional simulations of the cylinder with aspect ratio 8 and volume 30 $\mu \mathrm{L}$ - the three dimensional behaviour of the flow field inside the cylinder of aspect ratio 3 is lost. This is to be expected due to the constraints imposed by the two dimensionality of the geometry for this simulation. Thus, one can conclude that three dimensional 
(a)

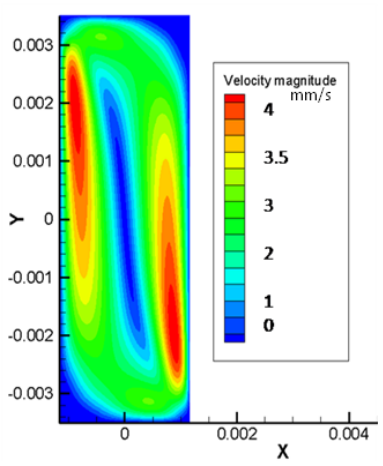

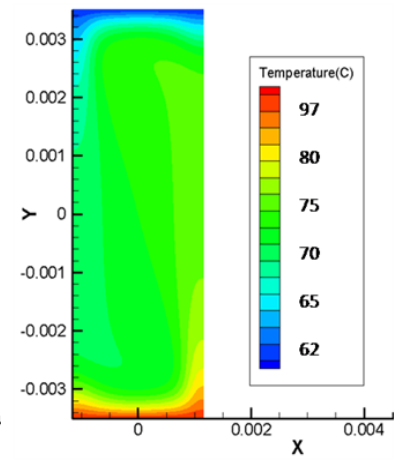

(b)

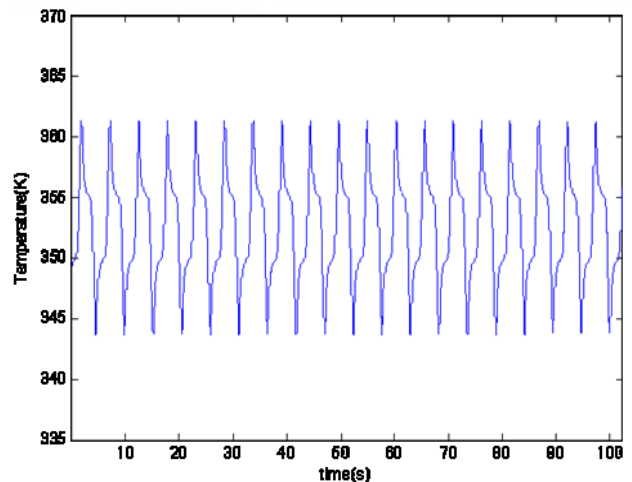

Fig. 15(a) Contour plots of velocity and temperature for two dimensional simulation of cylinder with aspect ratio 3. Fig. 15(b) Plot of temperature versus time for a typical streamtrace in the cylinder with aspect ratio 3.

computational fluid dynamics simulations are needed to capture the real behavior of flow inside a cylindrical cavity.

\section{EXPERIMENTS IN REDESIGNED REACTOR}

A new series of plastic cartridges were constructed incorporating cylindrical reaction chambers $1.98 \mathrm{~mm}$ in dia. and $6.02 \mathrm{~mm}$ high $(h / d=3.04$; volume $=18.5 \mu \mathrm{L})$. We then used these reactors in conjunction with the instrument setup shown in Fig. 5(b) to perform a series of new PCR amplifications of a 295 bp $\beta$-actin target from a human genomic DNA template (TaqMan $\beta$-actin PCR kit; Applied Biosystems). A typical 100 $\mu \mathrm{L}$ reaction mixture contained $10 \mu \mathrm{L}$ of $10 \mathrm{x}$ buffer solution, $6 \mu \mathrm{L}$ of $25 \mathrm{mM} \mathrm{MgCl}_{2}, 10$ $\mu \mathrm{L}$ of dNTPs (2 mM each), $41.2 \mu \mathrm{L}$ of DI water, $10 \mu \mathrm{L}$ of $\beta$-actin probe, $10 \mu \mathrm{L}$ of $\beta$ actin forward primer, $10 \mu \mathrm{L}$ of $\beta$-actin reverse primer, $2 \mu \mathrm{L}$ of human genomic template DNA $(10 \mathrm{ng} / \mu \mathrm{L})$ and $0.8 \mu \mathrm{L}$ of KOD DNA polymerase $(2.5$ units/ $\mu \mathrm{L})$. The enzyme, 
buffer (buffer \#1), $\mathrm{MgCl}_{2}$, and dNTPs were supplied with the KOD DNA Polymerase kit (cat. no. 71085-3; Novagen). The primers, probe, and template DNA were supplied with the TaqMan $\beta$-actin Control Reagents kit (cat. no. 401846; Applied Biosystems). Before loading reagents, the lower surface of the PCR chamber was sealed using a thin sheet of aluminum tape (cat. no. PCR-AS-200; Axygen, Inc.). The reactor wells were then rinsed with a $10 \mathrm{mg} / \mathrm{mL}$ aqueous solution of bovine serum albumin (cat. no. A2153; Sigma-Aldrich) followed by Rain-X Anti-Fog (SOPUS Products). We found that these rinsing steps helped to mimize adsorption at the sidewalls that may otherwise inhibit the reaction while also enhancing surface wettability so that reagents can be loaded without trapping air pockets inside the reactor. Reagents were then pipetted into the reactor wells and the upper surface was sealed with FEP Teflon tape (cat. no. 7562A17; McMasterCarr).

Before beginning the reaction, both aluminum blocks were pre-heated to the desired upper and lower surface temperatures The plastic reactor loaded with PCR reagents was then sandwiched between the aluminum heating blocks, and the assembly was quickly clamped together using nylon screws. This procedure minimized thermal transients so that the convective flow field could reach a steady state in $\sim 1 \mathrm{~min}$, as evident by the fact that the temperature readings from both aluminum blocks became stable after this period of time. In the reactions reported here, the upper and lower surfaces were maintained at 53 and $96^{\circ} \mathrm{C}$, respectively. After the reaction proceeded for the desired time, the heaters were switched off and the lower (hot) surface of the device was rapidly cooled by placing it on top of a chilled metal block. This protocol allowed 
the flow (and the PCR) to be stopped within a few seconds so that the total reaction time could be controlled with reasonable accuracy. The plastic reactor was then removed and the products were pipetted out of the wells for subsequent analysis by agarose gel electrophoresis ( $2 \mathrm{wt} \%$ gel (cat. no. 161-3107; Bio-Rad) with 1x TAE running buffer (cat. no. 141-0743; Bio-Rad)). Fluorescently stained DNA samples were prepared containing $2 \mu \mathrm{L}$ 100x SYBR Green I solution (Invitrogen/Molecular Probes), $2 \mu \mathrm{L}$ DNA sample, $2 \mu \mathrm{L}$ 6x Orange Loading Dye (Fermentas), and $4 \mu \mathrm{L}$ TAE buffer. Samples were run at $60 \mathrm{~V}$ for $1 \mathrm{~h}$ with a $100 \mathrm{bp}$ DNA ladder sizing marker (cat. no. 170-8202; BioRad). These reactions were also performed in a cylinder with aspect ratio $9(h=$ $15.9 \mathrm{~mm}, d=1.75 \mathrm{~mm}$, volume $=38.2 \mu \mathrm{L})$.

Remarkably, it was observed that it was possible to routinely achieve amplification of the correct target product in only 10 min using this new reactor design (Fig. 16a), while the same reaction required atleast $20 \mathrm{~min}$ in the cylinder with aspect ratio 9 (Fig. 16b). It is likely that the reaction is occurring even faster because the $10 \mathrm{~min}$ time includes 1-2 min to start up and shut off our heating device. Based on these results, we believe the incubation time previously observed $h / d=9$ was a manifestation of the closed streamlines that may trap fluid elements in unfavorable thermal trajectories for an extended period of time. These results suggest that it is possible to achieve orders of magnitude enhancements in cycling time ( 20 s per cycle), a capability that is an inherent feature of the underlying physics of convective flow in cavity-based systems. We also note that subsequent fluorescence analysis revealed that a signal from the TaqMan probe was detectable in all samples where amplification was successfully performed (Fig. 17). 
This verifies that cavity-based convective reactors can be used to perform real-time PCR. Another observation is that while a single product was obtained in the case
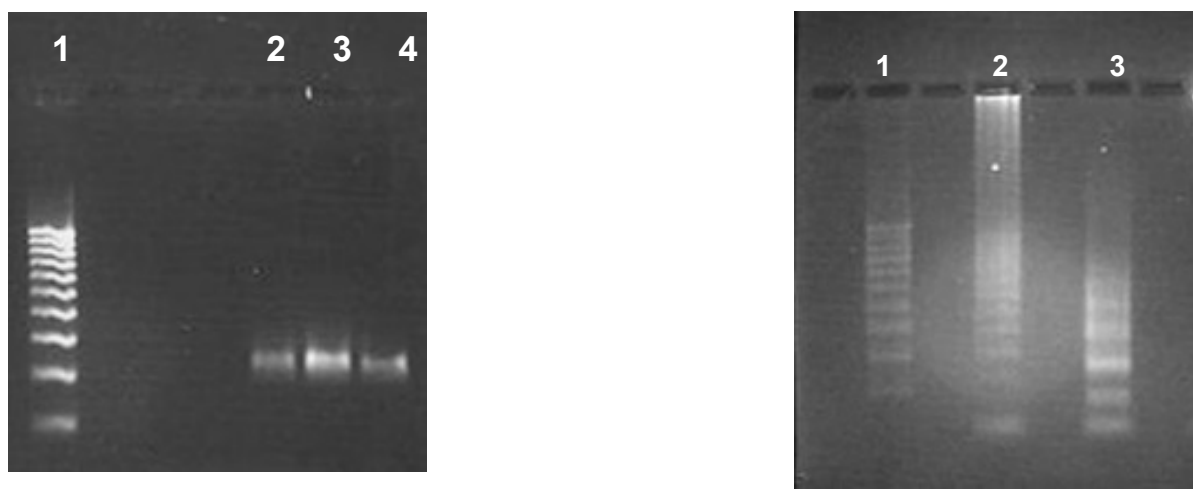

Fig. 16(a) Results from the cylinder with aspect ratio 3. Lane 1: $100 \mathrm{bp}$ ladder, Lanes 24: PCR product from 3 parallel reactions in the multiwell device. Fig. 16(b) Results from the cylinder with aspect ratio 9. Lane 1: 100 bp ladder, Lanes 2, 3: PCR product from 2 parallel reactions in the multiwell device.

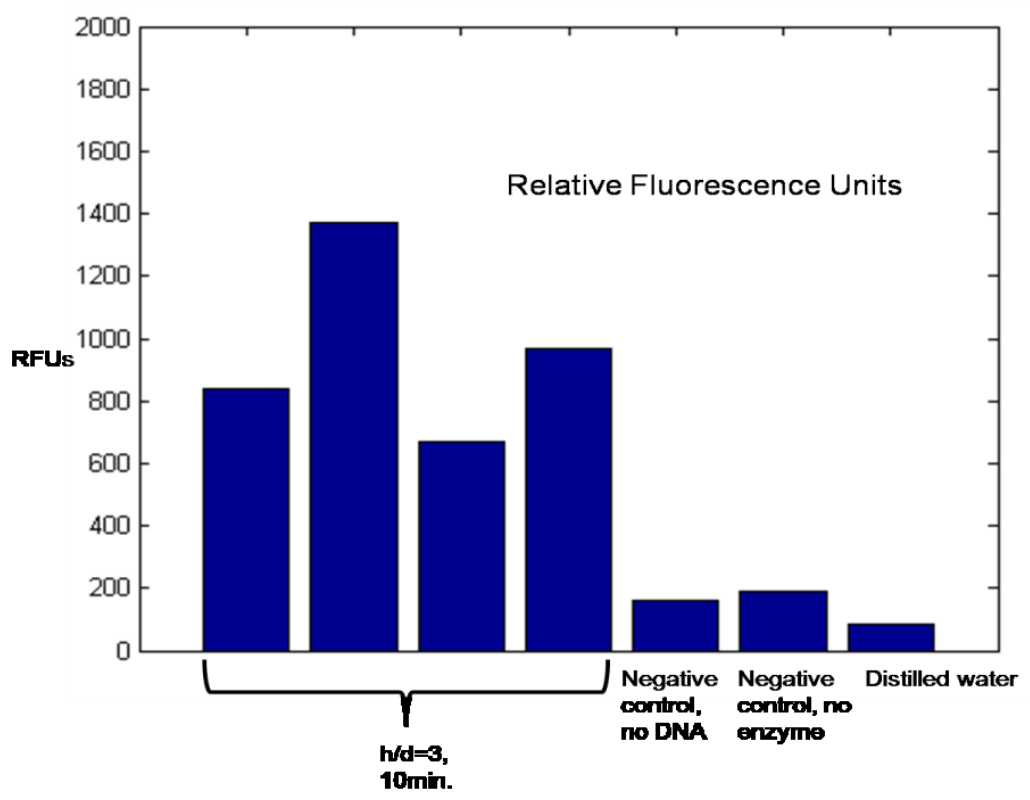

Fig. 17 Fluorometer analysis of PCR product from convective device with aspect ratio 3. 
of the cylinder with aspect ratio 3, the reaction in the cylinder with aspect ratio 9 often produced a series of products, that included unwanted DNA strands unrelated to the target. In order to explain this phenomenon, a conjecture was made that in the cylinder with aspect ratio 9, fluid particles seemed to be circulating in closed loop trajectories making the flow more two dimensional than three dimensional. This results in reagents being exposed to different sets of temperature ranges. For example, a few reagents may be exposed to temperatures between $96^{\circ} \mathrm{C}$ and $53^{\circ} \mathrm{C}$ (i.e. circulating completely from top to bottom), while other reagents may be exposed to temperatures between $91^{\circ} \mathrm{C}$ and $58^{\circ} \mathrm{C}$ (i.e. following trajectories confined near the center of the cylinder). Consequently, PCR reagents may be exposed to a mutiplicity of temperature histories, leading to the production of spurious DNA strands.

In order to test this hypothesis, a set of reactions were performed in a conventional thermocycler by allowing reagents to undergo reactions within temperature

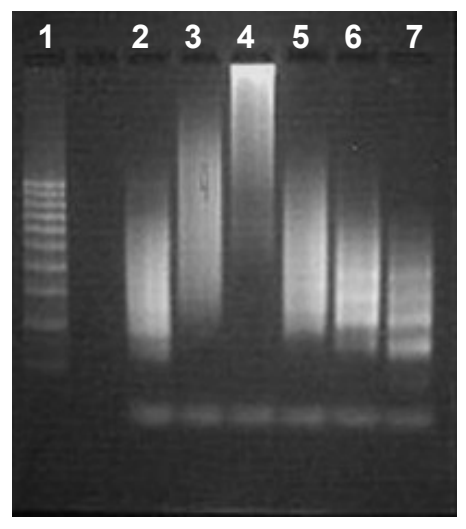

Fig. 18 Results from PCR performed in the thermocycler.

Lane 1: 100 bp ladder, Lane 2: $91.6{ }^{\circ} \mathrm{C} / 58.4{ }^{\circ} \mathrm{C}$, Lane 3: 92.5

${ }^{\circ} \mathrm{C} / 57.5^{\circ} \mathrm{C}$, Lane $4: 93.7{ }^{\circ} \mathrm{C} / 56.3{ }^{\circ} \mathrm{C}$, Lane 5: $94.9{ }^{\circ} \mathrm{C} / 55.1{ }^{\circ} \mathrm{C}$, Lane 6: $96{ }^{\circ} \mathrm{C} / 54{ }^{\circ} \mathrm{C}$, Lane $7: 96.5{ }^{\circ} \mathrm{C} / 53.5{ }^{\circ} \mathrm{C}$. 
ranges of $\left(96{ }^{\circ} \mathrm{C}-53{ }^{\circ} \mathrm{C}\right)$ and $\left(91^{\circ} \mathrm{C}-58{ }^{\circ} \mathrm{C}\right)$ for 30 cycles. It can be seen from Fig. 18 that the different ranges of reaction temperature give rise to products in different size ranges. Thus, the multiple temperature profiles produced in the cylinder with aspect ratio 9 are likely to generate multiple unwanted products. This result is in contrast to the cylinder with aspect ratio 3 which enhances mixing of the reactants across pathlines, allowing a larger fraction of reagents to experience favorable temperature profiles. The reactants also spend a greater amount of time in the extension regime. This favorable combination of effects enables DNA replication to proceed more rapidly in the cylinder with aspect ratio 3 .

\section{COMPUTATIONAL FLUID DYNAMICS SIMULATIONS OF PCR GEOMETRIES}

Computational fluid dynamic simulations were also performed for the cylinders used in the PCR experiments. The height and diameter for the cylinder with aspect ratio 3 was $6.02 \mathrm{~mm}$ and $1.98 \mathrm{~mm}$ (volume $18.5 \mu \mathrm{L}$ ), while that for the cylinder with aspect ratio 9 was $15.9 \mathrm{~mm}$ and $1.75 \mathrm{~mm}$ (volume $33 \mathrm{ul}$ ). The top and bottom surfaces were simulated at constant temperature boundary conditions of $53{ }^{\circ} \mathrm{C}$ and $96{ }^{\circ} \mathrm{C}$. The sidewalls were simulated with insulated boundary conditions. Fig. 19a-19d shows the contour plots of temperature and velocity for the cylinder with aspect 3. Fig. 19e-19h shows the contour plots of temperature and velocity for the cylinder with aspect 9. Fig. 19i-191 shows the vector plots of velocity for the cylinder with aspect ratio 3 and 9 respectively. Fig. 20 and Fig. 21 show a typical streamtrace for the cylinders with aspect ratio 3 and 9 respectively. These results are similar to the ones obtained from cylinders 
(a) $h / d=3$

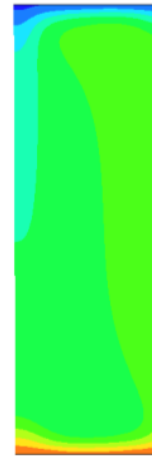

(e) $h / d=8$

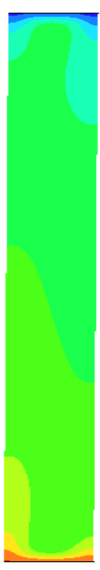

(i) $h / d=3$

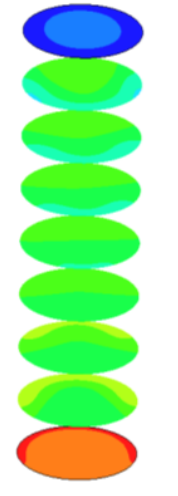

(b) $h / d=3$

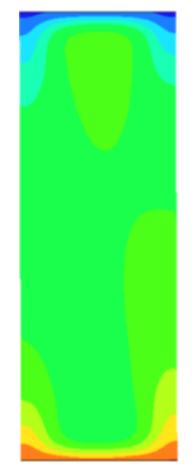

(f) $h / d=8$

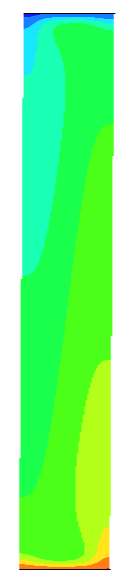

(j) $h / d=3$

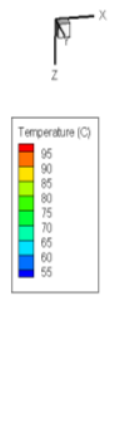

(c) $h / d=3$
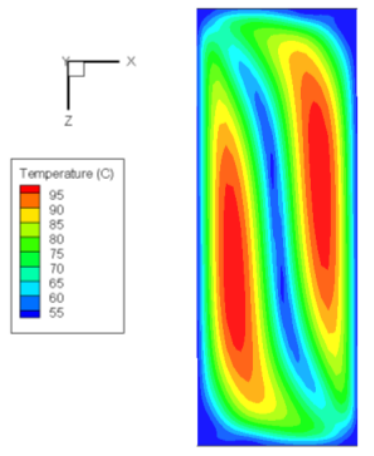

(g) h/d = 8

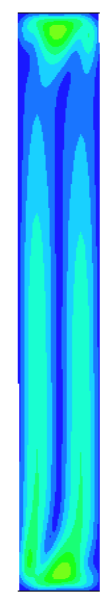

(k) $\mathrm{h} / \mathrm{d}=8$

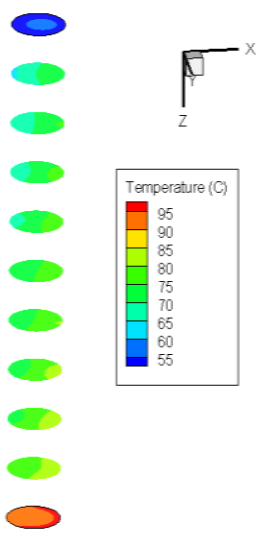

(d) $\mathrm{h} / \mathrm{d}=3$

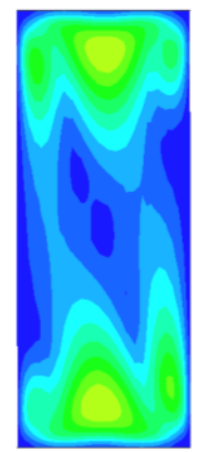

(h) h/d = 8
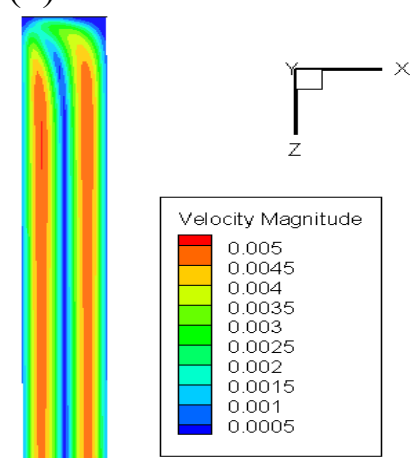

(1) $h / d=8$
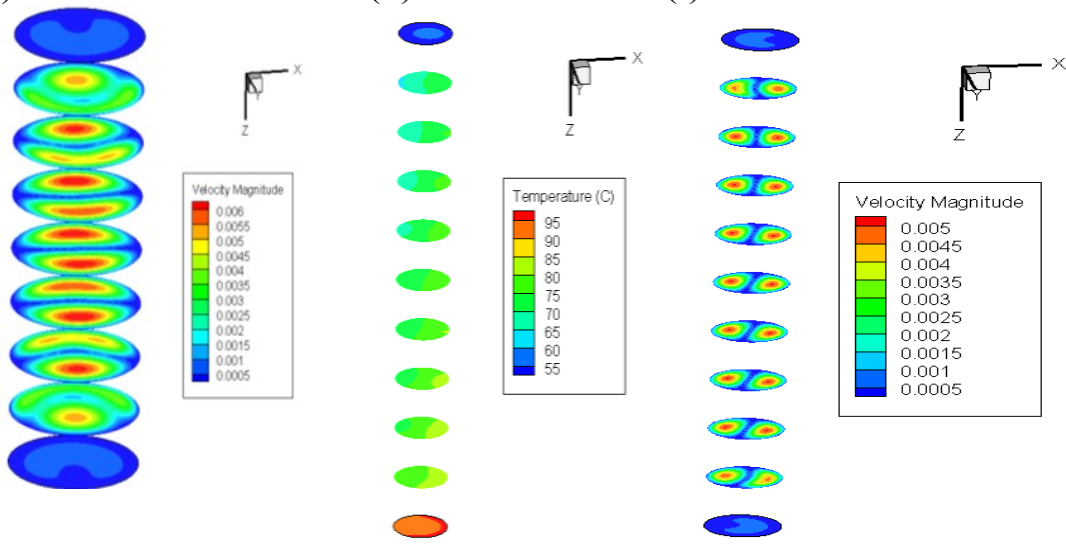

Fig. 19(a)-19(d) Contour plots of temperature and velocity in the XZ and YZ planes for cylinder with aspect ratio 3. Fig. 19(e)-19(h) Contour plots of temperature and velocity in the $\mathrm{XZ}$ and $\mathrm{YZ}$ planes for cylinder with aspect ratio 8 . Fig. 19(i)-19(1) Contour plots of temperature and velocity in the XY planes for cylinder with aspect ratio 3 and 8 , respectively. 
with aspect ratio 3 and 8 , with a constant volume of 30ul, i.e. the flow in the cylinder with aspect ratio 3 seems disordered, while the flow for the cylinder with aspect ratio 9 seems more periodic. The results from the simulations and the PCR experiments indicate

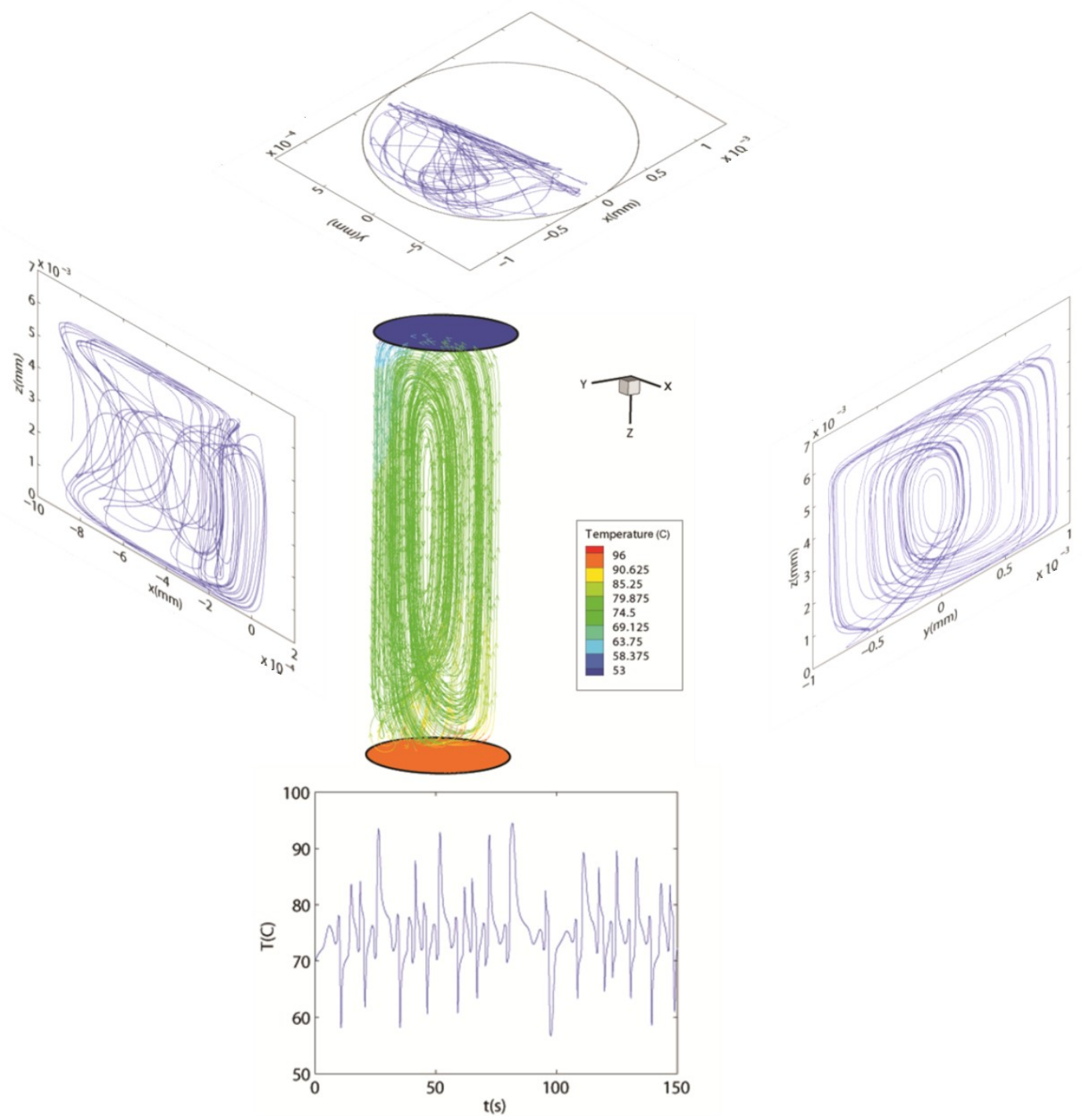

Fig. 20 Typical streamtrace in cylinder with aspect ratio 3. The temperature profile shows a disordered behavior. 
that the flow field in the cylinder affects the rate of reaction for PCR. This has been studied in greater detail in the next chapter.
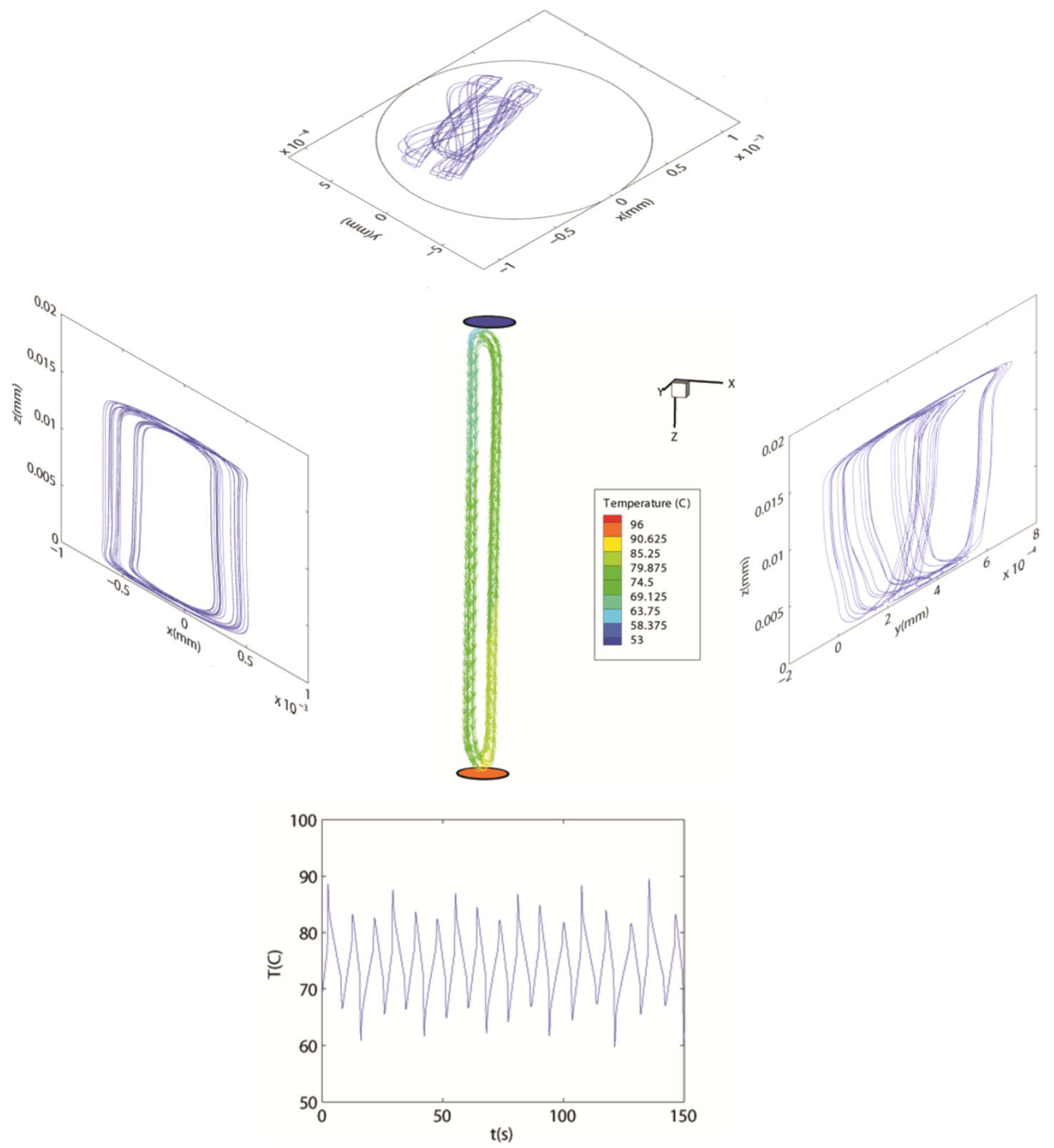

Fig. 21 Typical streamtrace in cylinder with aspect ratio 9. The temperature profile shows a more organized behavior. 


\section{CHAPTER III}

\section{NUMERICAL SIMULATION OF CHEMICAL REACTION}

\section{INTRODUCTION}

It was established that the cylinder with aspect ratio 3 (Volume $18.5 \mu \mathrm{L}$ ) appeared to have faster reaction time compared to the cylinder with aspect ratio 9 (Volume 33ul). Further analysis of this phenomenon is possible by coupling the flow to the biochemical reactions that occur during PCR. In order to compute the evolution of the DNA concentration fields in the convective flow reactor, it is necessary to solve the concentration evolution equations proposed by Dorfman et al [28], where they solved the chemical kinetics equations in a two dimensional reactor. With our current three dimensional FLUENT simulations, it should be possible to include the chemical reactions; however, we found that it was not possible to setup a reaction with thermally localized steps in FLUENT. Following similar work in a two dimensional reactor (Dorfman et al [28]) in COMSOL Multiphysics, we found that we could not obtain a converged solution for a three dimensional reactor due to an instability driven nature of the flow. We have now tried to simplify the problem by noting that PCR is a complex reaction which occurs in three different steps - we consider a simplified kinetic model to understand the main features of the flow. We also note that the temperature vs. time profile obtained from the particle paths essentially provides us with the concentration following the fluid element - this information can be used with the initial conditions for concentration to solve for the evolution of concentrations for a given trajectory. 


\section{COUPLED FLOW AND CHEMICAL REACTIONS}

It has recently been shown that many important features of convective PCR can be captured by a simplified framework expressed in terms of kinetics associated with (1) denaturing: dsDNA $\rightarrow$ 2ssDNA (rate constant $k_{d}$ ); (2) annealing: ssDNA $\rightarrow$ aDNA (rate constant $k_{a}$ ); and (3) extension: aDNA $\rightarrow$ dsDNA (rate constant $k_{e}$ ) $[28,29]$. These expressions serve as inputs to a dimensional reaction-diffusion equation [30,31]:

$$
\frac{\partial c_{i}{ }^{\prime}}{\partial t^{\prime}}+v^{\prime} \cdot \nabla^{\prime} c_{i}{ }^{\prime}=D \nabla^{\prime 2} c_{i}{ }^{\prime}+r_{i}^{\prime}
$$

where $D$ is the mass diffusion coefficient and the rates of change in species concentration $r_{i}{ }^{\prime}$ take the form $k_{i}{ }^{\prime} c_{i}{ }^{\prime}$. In order to non-dimensionalize this equation, we scale position by the cylinder height $h\left(x=x^{\prime} / h, y=y^{\prime} / h, z=z^{\prime} / h\right)$, velocity by the magnitude $\bar{u}$ averaged over the flow trajectory $\left(u=u^{\prime} / \bar{u}, v=v^{\prime} / \bar{u}, w=w^{\prime} / \bar{u}\right)$, reaction rate by a characteristic constant $k^{*}\left(k=k^{\prime} / k^{*}\right)$, concentration with a characteristic quantity $c^{*}\left(c_{i}=c_{i}{ }^{\prime} / c^{*}\right)$, and time with a characteristic timescale $t^{*}\left(t=t^{\prime} / t^{*}\right)$. Substituting these quantities into Eq. (7) gives (the quantities $c^{*}$ cancel out).

$$
\frac{\partial c_{i}}{\partial t}+\left(\frac{\bar{u} t^{*}}{h}\right) v \cdot \nabla c_{i}=\left(\frac{D t^{*}}{h^{2}}\right) \nabla^{2} c_{i}+\left(k^{*} t^{*}\right) k_{i} c_{i}
$$

This scaling yields three groups of dimensionless parameters $\left(\bar{u} t^{*} / h, D t^{*} / h^{2}, k^{*} t^{*}\right)$, the relative magnitudes of which are largely determined by the characteristic timescale $t^{*}$. There are several possibilities for the definition of $t^{*}:$ it can be linked to the average velocity $(\bar{u})$, the characteristic reaction rate $\left(k^{*}\right)$, thermal diffusivity $(\alpha)$, mass diffusivity $(D)$, or kinematic viscosity $(v)$. In order to guide our choice, we considered a typical 
streamtrace in the cylindrical reactor at $h / d=3($ volume $=18.5 \mu \mathrm{L})$ with a characteristic value $k^{*}=5.564 \mathrm{~s}^{-1}$ obtained from solving the kinetic model at $D a^{C}=10$ (see next section). We assume that all three species (dsDNA, ssDNA and aDNA at dimensionless concentrations of $c_{d s}, c_{s s}$, and $c_{a}$, respectively) have an identical mass diffusivity $D=10^{-7}$ $\mathrm{cm}^{2} / \mathrm{s}$, and fluid properties are evaluated at $95{ }^{\circ} \mathrm{C}$. Table 2 summarizes the values for the three abovementioned dimensionless groups obtained under these conditions.

Table 2. Comparison of different choices for the characteristic timescale $t^{*}$ on the dimensionless groups appearing in equation 8 .

\begin{tabular}{|c|c|c|c|}
\hline $\boldsymbol{t}^{*}(\mathbf{s})$ & $\overline{\boldsymbol{u}} \boldsymbol{t}^{*} / \boldsymbol{h}$ & $\boldsymbol{D} \boldsymbol{t}^{*} / \boldsymbol{h}^{2}$ & $\boldsymbol{k} \boldsymbol{t}^{*}$ \\
\hline$h^{2} / D=3.620 \times 10^{6}$ & $1.986 \times 10^{6}$ & 1 & $2.016 \times 10^{7}$ \\
\hline$h^{2} / \alpha=244.8$ & 134.2 & $6.755 \times 10^{-5}$ & 1362 \\
\hline$h^{2} / v=116.8$ & 64.02 & $3.223 \times 10^{-5}$ & 649.9 \\
\hline $1 / k=0.1800$ & 0.0985 & $4.959 \times 10^{-8}$ & 1 \\
\hline $\boldsymbol{h} / \bar{u}=\mathbf{1 . 8 2 4}$ & $\mathbf{1}$ & $\mathbf{5 . 0 3 3 \times \mathbf { 1 0 } ^ { - 7 }}$ & $\mathbf{1 0 . 1 5}$ \\
\hline
\end{tabular}

Several key observations can now be made. First, it is clear that in all cases (except the trivial one where $h^{2} / D$ is the basis) the dimensionless group $D t^{*} / h^{2}$ associated with the Laplacian term is considerably smaller than the others, leading to the conclusion that this term can be neglected in our evaluation of the kinetic model. Secondly, we see that using either mass diffusivity, thermal diffusivity, or kinematic viscosity as a basis to obtain a characteristic timescale yields dimensionless groups associated with the convective $\left(\bar{u} t^{*} / h\right)$ and reaction $\left(k^{*} t^{*}\right)$ terms that are of much greater magnitude than the term associated with the time rate of change in species concentration, which is of order 
unity. Excluding these choices leaves the reaction rate and flow velocity as potential candidates for the characteristic timescale. Now, we note that our analysis uses temperature versus time data corresponding to particle paths obtained from simulations of the 3D velocity field as inputs to the kinetic model, ultimately yielding species concentrations $c_{i}$ following a fluid element. Since the convective term in Eq.(8) is directly linked to the fluid motion, it is reasonable to expect that the dimensionless group associated with this term would be better represented using a timescale based on the flow velocity rather than the chemical reaction rate. Based on these considerations, we therefore chose to scale the time variable by $h / \bar{u}$, yielding the following form of the dimensionless concentration evolution equation.

$$
\frac{\partial c_{i}}{\partial t}+v \cdot \nabla c_{i}=\frac{D c_{i}}{D t}=\frac{1}{P e} \nabla^{2} c_{i}+D a^{c} r_{i}
$$

Here, the Péclet number is defined as $P e=h \bar{u} / D$ and the convective Damköhler number is defined as $D a^{C}=k^{*} h / \bar{u}$. The subscripts $i$ denote the species ssDNA, aDNA, and dsDNA, with respective rates of change $r_{i}$ given by:

$$
\begin{aligned}
& r_{s s}=2 \kappa_{d} f_{d}(\mathbf{x}, t) c_{d s}-\kappa_{a} f_{a}(\mathbf{x}, t) c_{s s} \\
& r_{a}=\kappa_{a} f_{a}(\mathbf{x}, t) c_{s s}-\kappa_{e} f_{e}(\mathbf{x}, t) c_{a} \\
& r_{d s}=\kappa_{e} f_{e}(\mathbf{x}, t) c_{a}-\kappa_{d} f_{d}(\mathbf{x}, t) c_{d s}
\end{aligned}
$$

The initial conditions for the dimensionless species concentrations are obtained by assuming that all DNA is double-stranded at time $t=0$, yielding $c_{d s}(t=0)=1, c_{s s}(t=$ $0)=c_{a}(t=0)=0$. The parameters $\kappa_{\mathrm{i}}$ denote dimensionless reaction rates for the 
denaturing, annealing, and extension steps given by $\kappa_{\mathrm{i}}=k_{i} / k^{*}$. Following the analysis of Yariv et al [29], we assume values of $\kappa_{\mathrm{a}}=\kappa_{\mathrm{d}}=1$ and $\kappa_{\mathrm{e}}=0.5$. Mapping functions $f_{i}$ are applied to ensure that each reaction step (denaturing, annealing, extension) is localized within its respective temperature zone. These functions take the form of a Gaussian profile, shown in Eq.(13),

$$
f_{i}(x, t)=f_{i}(\theta)=\exp \left[-\frac{\left(\theta-\theta_{i}\right)^{2}}{2 \sigma_{i}^{2}}\right]
$$

expressed in terms of a dimensionless temperature $\theta$ defined as

$$
\theta=\frac{\left(T-T_{t o p}\right)}{\left(T_{b o t t o m}-T_{t o p}\right)}
$$

where $T_{\text {top }}$ and $T_{\text {bottom }}$ are the temperatures at the top (cold) and bottom (hot) surfaces of the cylindrical reactor cavity. The parameter $\theta_{i}$ represents the dimensionless temperature corresponding to each step in the reaction (i.e., at $T=T_{i}$, where the $T_{i}$ correspond to

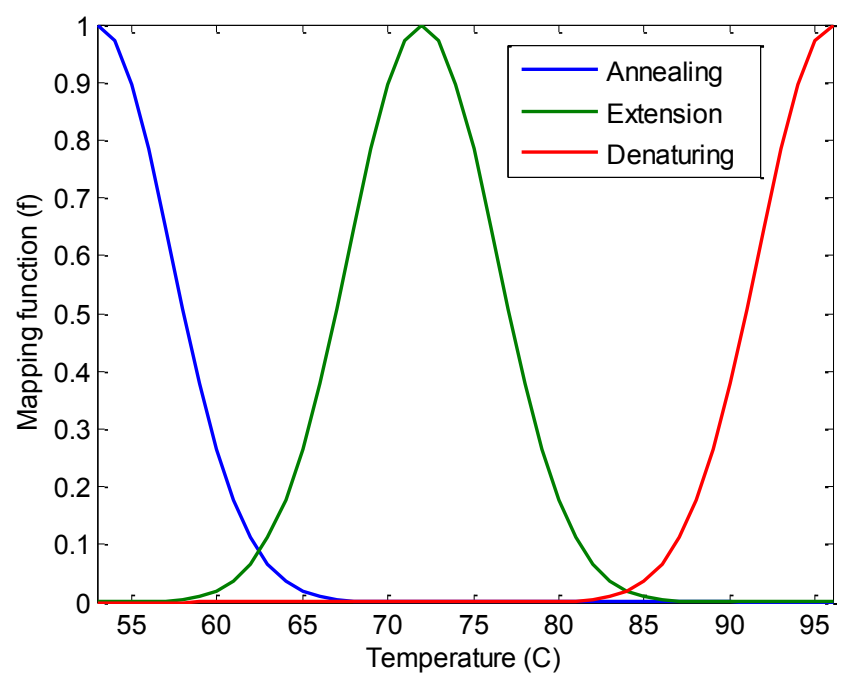

Fig. 22 Temperature distribution of mapping function $f$. 
denaturing $\left(T_{d}=96{ }^{\circ} \mathrm{C}\right)$, annealing $\left(T_{a}=53{ }^{\circ} \mathrm{C}\right)$, and extension $\left(T_{e}=72{ }^{\circ} \mathrm{C}\right)$ steps $)$. In our analysis the top and bottom surfaces of the cylindrical reactor are maintained at $T_{a}$ and $T_{d}$, respectively. The parameter $\sigma_{i}$ representing the standard deviation of reaction rate with respect to temperature was chosen to be 0.1 for all three reaction steps. This choice of parameters yields the mapping functions shown in Fig. 22. Using the results from the computational fluid dynamics simulations, one can then obtain the concentrations of the double stranded, annealed and single stranded DNA as a function of time. Plotting the logarithmic plot of the concentration of the double stranded DNA versus time and fitting a linear polynomial yields the rate at which the concentration of the double stranded DNA increases. The doubling time can be found by using Eq. (15):

$$
\tau=\ln (2) / \text { slope }
$$

\section{EVALUATING THE KINETIC MODEL}

We evaluated Eq.9-12 over a range $D a^{C}$ to establish an appropriate value for the characteristic reaction rate $k^{*}$ that could be consistently used in all calculations. The $h / d$ $=3$ geometry was adopted as a base case, where the velocity magnitude over the streamtrace (depicted in Fig. 20) was $\bar{u}=3.3 \mathrm{~mm} / \mathrm{s}$ and the cylinder height was $h=6.02$ mm. Under these conditions, we considered the doubling time of $27.3 \mathrm{~s}$ obtained at $D a^{C}$ $=10$ to be a reasonable order of magnitude representation of the observed DNA replication rate (based on our limited ability to infer kinetics from gel electrophoresis data), yielding $k^{*}=5.564 \mathrm{~s}^{-1}$ which is also consistent with available kinetic data [32]. This value, along with the characteristic velocities $\bar{u}=3.3$ and $3.7 \mathrm{~mm} / \mathrm{s}$ at $h / d=3$ and 
9, respectively, was maintained throughout all subsequent calculations. For example, applying the cylinder height $h=15.9 \mathrm{~mm}$ at $h / d=9$ yielded $D a^{C}=24$. A second important issue arises due to the characteristics of the streamtraces extracted from the CFD simulations for use as input to the kinetic model. While these data provide a consistent representation of flow trajectories within the reactor volume, it is difficult to choose two streamtraces that reach identical temperature extremes. In other words, the flow paths do not carry reagents all the way to the upper and lower bounding surfaces, but instead reach maximum values $\sim 1-5{ }^{\circ} \mathrm{C}$ below the denaturing extreme $\left(T_{d}=96{ }^{\circ} \mathrm{C}\right)$ and $\sim 1-5{ }^{\circ} \mathrm{C}$ above the annealing extreme $\left(T_{a}=53{ }^{\circ} \mathrm{C}\right)$. We found that this variability, although slight, could lead to incorrect conclusions regarding reaction kinetics compared among different reactor geometries by reflecting differences in temperature rather than differences in the flow field, an especially important point since we are examining individual streamtraces. We dealt with this issue by applying a minor correction $\delta \mathrm{T}$ to the temperature versus time data to ensure that the maximum and minimum temperatures in each streamtrace would reach the denaturing and annealing extremes (see Fig. 23). This correction involved computing the difference between (1) the maximum (or minimum) temperatures in the streamtrace dataset, and (2) the denaturing (or annealing) temperatures $\left(T_{d}=96{ }^{\circ} \mathrm{C}, T_{a}=53{ }^{\circ} \mathrm{C}\right)$. The denaturing correction factor $\left(\delta \mathrm{T}_{\mathrm{d}}\right)$ was then added to all temperature data greater than $T_{a v g}=\left(T_{d}+T_{a}\right) / 2$, and the annealing correction factor $\left(\delta \mathrm{T}_{\mathrm{a}}\right)$ was subtracted from all temperature data less than $T_{\text {avg. }}$. These corrections ensured that comparisons made between doubling times obtained from different streamtraces accurately reflect features of the corresponding flow profiles. 


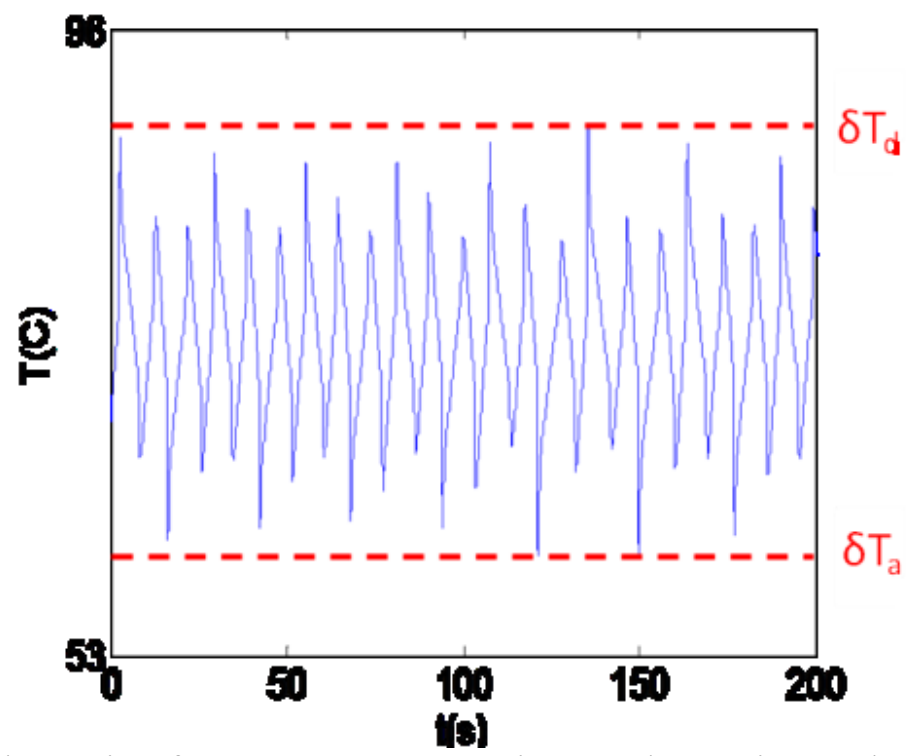

Fig. 23 Schematic of temperature correction used to estimate doubling time.

\section{RESULTS OF CHEMICAL REACTION KINETICS}

The temperature versus time data shown in Fig. 20 and 21 express a Lagrangian representation of the local environment surrounding a fluid element as it experiences advection along its flow trajectory, and can thus be applied directly to evaluate the rates of change associated with each individual species concentration $\mathrm{D} c_{i} / \mathrm{D} t$ using Eq. 9-12 (the 1/Pe term in Eq. (9) can be neglected under the conditions of interest here, where $P e$ $\sim 10^{7}$ ). These calculations yield kinetic data that follow a characteristic trend of exponential growth in dsDNA concentration, from which a doubling time can be extracted to yield a parameter representing the timescale associated with execution of a complete temperature cycle. We used this analysis to compare doubling times corresponding to representative streamtraces at $h / d=3$ and 9 (Fig. 24), where thermal profile data were scaled to ensure that the maximum and minimum temperatures 
encountered along each flow trajectory exactly reached the reactor's denaturing and annealing extremes $\left(T_{d}=96{ }^{\circ} \mathrm{C}\right.$ and $T_{a}=53{ }^{\circ} \mathrm{C}$, respectively). Applying characteristic parameters corresponding to the reactor geometries studied in Figs. 20 and 21 yields $D a^{C}$ $=10$ and 24 at $h / d=3$ and 9 , respectively. Based on this analysis, the doubling time at $h / d=9(11.6 \mathrm{~s})$ is predicted to be much more rapid than at $h / d=3(27.3 \mathrm{~s})$, sharply contradicting our experimental observation that the reaction proceeds at least twice as fast at $h / d=3$. This trend is maintained even when the thermal profiles are perturbed away from optimal conditions by scaling the maximum and minimum temperatures encountered along each streamtrace so that they reach $T_{d}=93{ }^{\circ} \mathrm{C}$ and $T_{a}=56{ }^{\circ} \mathrm{C}$ (i.e., $\delta T$ $=3{ }^{\circ} \mathrm{C}$ away from the ideal temperature extremes, see inset of Fig. 23), albeit with correspondingly lower doubling times (26.2 and $52.8 \mathrm{~s}$ at $h / d=9$ and 3 , respectively). The striking discrepancy between experimental results (showing that the reaction proceeds much faster at $h / d=3$ than at $h / d=9$ ) and kinetic simulations (which predict the opposite) can be explained by recalling that our implementation of the kinetic model only considers individual isolated flow trajectories. Evidently, the chaotic flow field's ability to collectively expose a large fraction of the reagent volume to favorable thermal conditions at $h / d=3$ is highly desirable, counterintuitively suggesting that while individual flow trajectories at $h / d=9$ may appear to be more favorable for PCR, the chaotic nature of the flow field at $h / d=3$ ultimately dominates reaction kinetics on a global scale. Exploring a broader range of conditions reveals that doubling times decrease as $D a^{C}$ increases at both $h / d=3$ and 9 , consistent with the predictions of Dorfman et al [28]. The close agreement among data from both geometries, nearly 


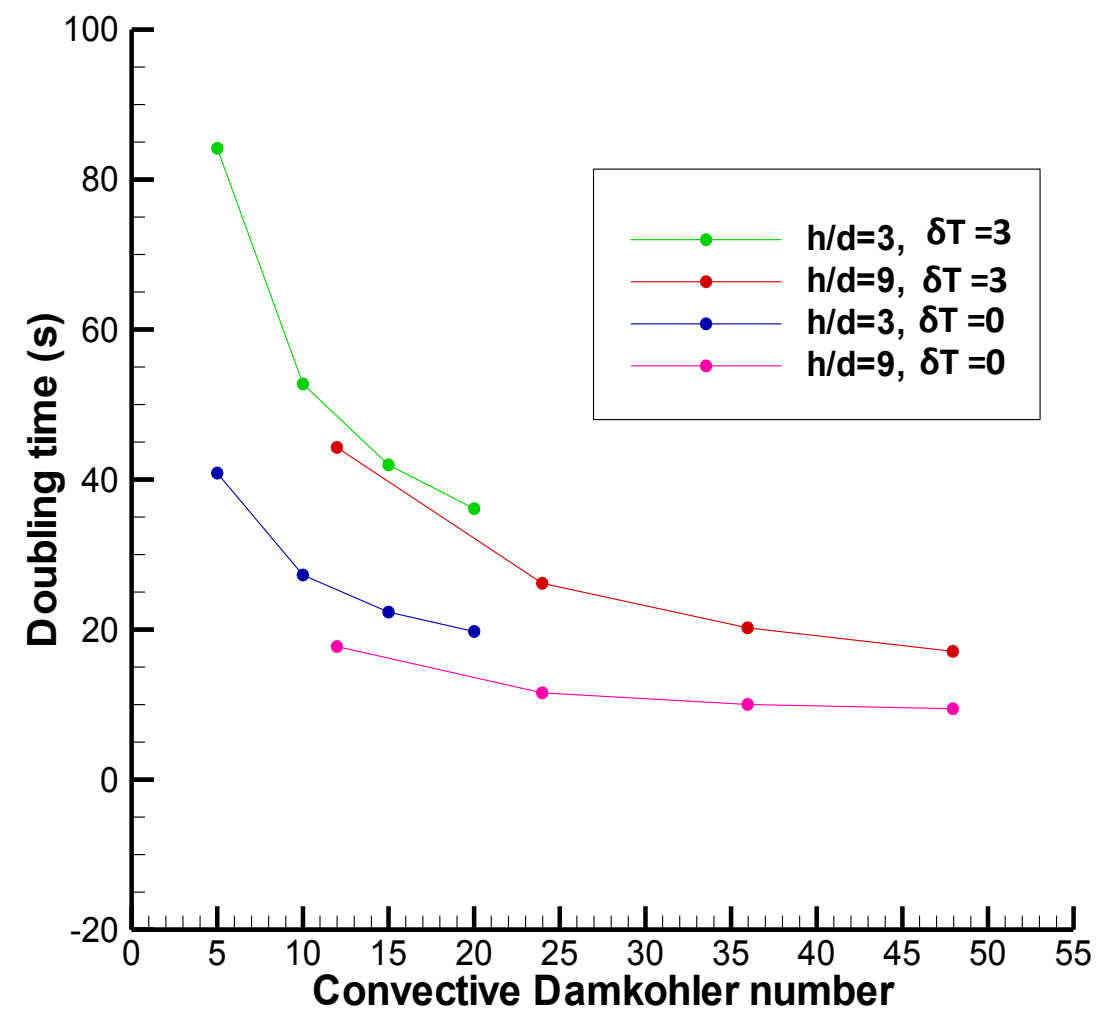

Fig. 24 Doubling times as a function of Damköhler number.

overlapping in the range where their respective $D a^{C}$ values coincide, suggests a fundamentally similar interplay between flow and reaction.

Extending this analysis to cylinders of different aspect ratios, with a constant volume of $30 \mu \mathrm{l}$, yields an interesting trend, as shown in Fig. 25-26. Here, a temperature correction of $0{ }^{\circ} \mathrm{C}$ was applied. From Fig. 25, one can notice that the doubling decreases as the Damköhler number increases for all the aspect ratios. Though the behavior is different for each aspect ratio, which is to be expected given that the flow profile is dependent on the aspect ratio, one can notice a power law profile emerging from the 
logarithmic plot of the Damköhler number versus the doubling time, as shown in Fig. 26. This behavior seems to indicate that the Damköhler number plays a vital role in determining the rate of a typical PCR reaction.

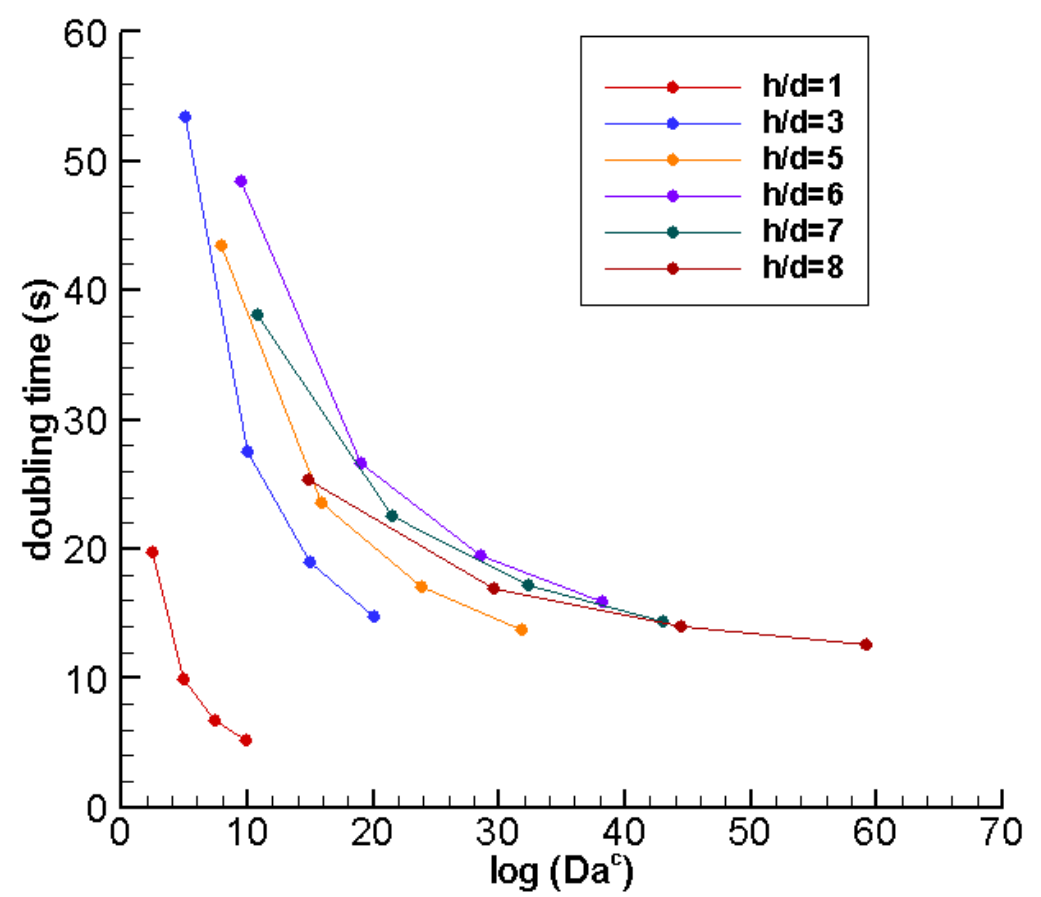

Fig. 25 Variation of the doubling time as a function of Damköhler number. 


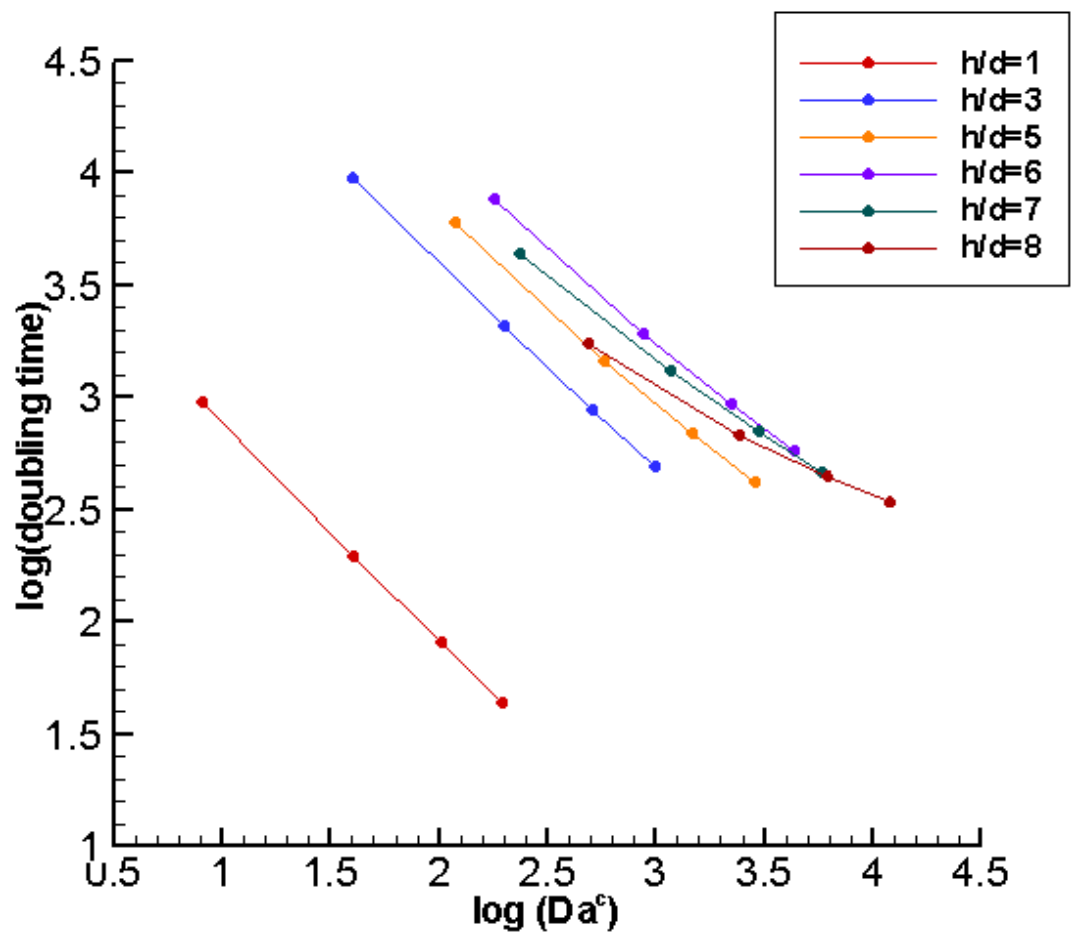

Fig. 26 Plot of the doubling time vs. Damköhler number on the log scale. 


\section{CHAPTER IV}

\section{QUANITFYING CHAOS IN CONVECTIVE FLOWS}

\section{INTRODUCTION}

It has been established by the use of the streamtraces from the computational fluid dynamics simulations of PCR geometries driven by Rayleigh-Bénard convection, that the fluid in the cylinder with aspect ratio 3 seems to be exhibiting symptoms of chaos, while the cylinder with aspect ratio 9 seems to exhibit a more ordered flow. We have used this hypothesis to perform PCR reactions in these geometries and have proven that indeed, due to the effect of chaos in the cylinder with aspect ratio 3, we have been able to perform the PCR reaction in under $10 \mathrm{~min}$, as compared to $20 \mathrm{~min}$ for the cylinder with aspect ratio 9. Through the use of the simulations of the chemical kinetics of the individual PCR reactions using a single streamtrace, we have shown that while the simulations predict lower doubling times for the cylinder with aspect ratio 9, in practice, the opposite is true - this is due to the fact that we considered a single streamtrace which does not reflect the ability of a chaotic flow to promote mixing within the fluid. Thus, it is necessary to be able to measure the chaotic nature of a flow field - this is accomplished in this chapter through the use of Poincare maps and correlation dimensions, thus providing qualitative and quantitative means of measuring chaos. We also illustrate the transitions between flows for different values of the aspect ratio and Rayleigh numbers, varying from axi-symmetric convective flow to non-axisymmetric flow, all the way to unsteady flow. 


\section{POINCARÉ SECTIONS}

It is possible to study the extent of "mixing" or exchange among flow trajectories by using Poincaré maps. A Poincaré section for a three dimensional trajectory is defined as the collection of points obtained when this trajectory pierces a chosen plane. Poincaré maps can be derived using the velocity field of the trajectory. It can be readily understood that if a trajectory exhibits a periodic closed path, then the Poincaré section will show distinct islands corresponding to the three dimensional trajectory; however, if the trajectory exhibits no periodicity, then the Poincaré sections will contain a disordered collection of points, which is an indication of chaos [33].

In the current study, Poincare maps were obtained by finding the location of the point where a given streamtrace pierces the mid-plane of the cylinder along its height. The characteristics of the flow trajectories for the reactor geometries used to perform PCR at aspect ratio 3 and 9 respectively were visualized using Poincaré maps, shown in Fig. 27(a) and Fig. 27(c). This analysis shows that for the cylinder with aspect ratio 9, the fluid elements follow tightly closed paths whose loci generate distinct KolmogorovArnold-Moser (KAM) curves. Consequently, reagents are exposed to a thermal profile characterized by quasi-periodic oscillation between upper and lower extremes, as seen when temperature versus time is plotted following a fluid element. A much different flow field emerges at $h / d=3\left(R a=1.45 \times 10^{6}\right.$; Fig. 27(b) and Fig.27(d)), appearing disordered in the sense that fluid elements follow complex 3D trajectories that do not produce well-defined KAM boundaries in the Poincaré map but instead yield a broadly distributed pattern consistent with the emergence of chaos [33]. These effects disrupt the 
(a)

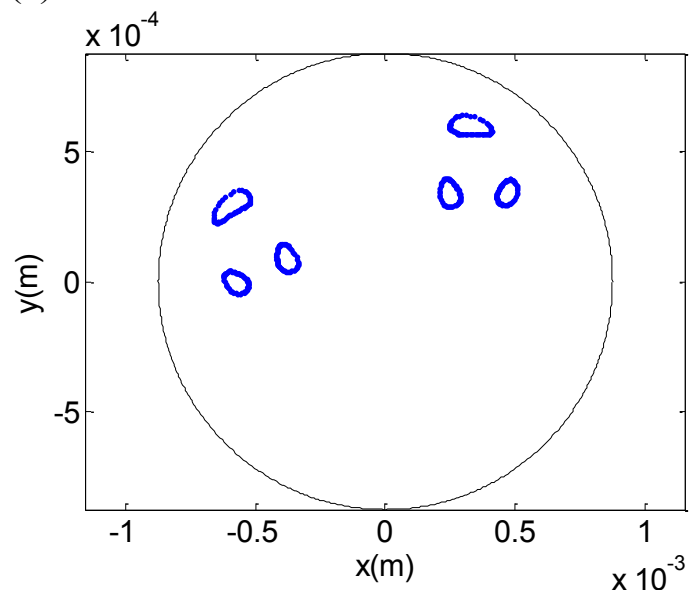

(c)

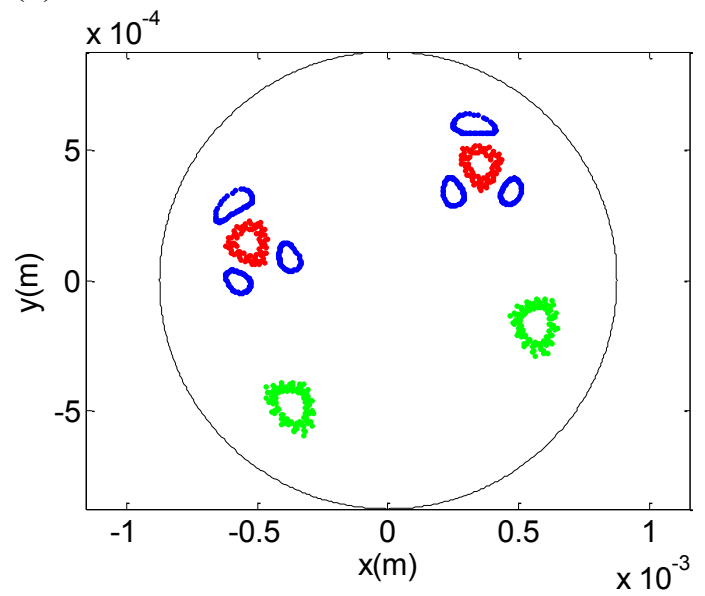

(b)

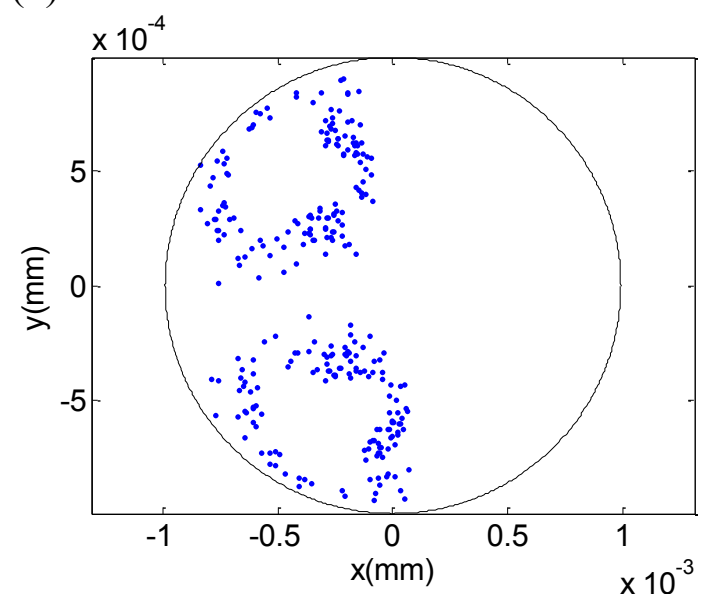

(d)

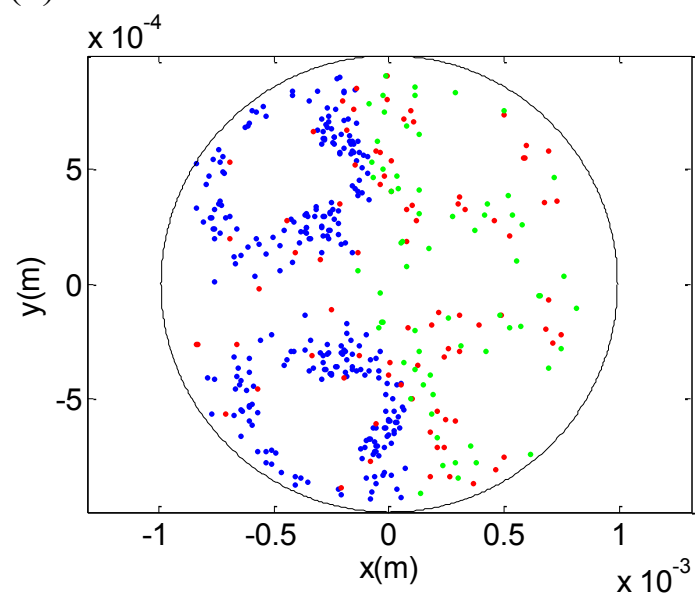

Fig. 27(a)-(b) Poincaré maps for a single streamtrace for cylinders with aspect ratio 9 and 3 respectively. Fig. 27(c)-(d) Poincaré maps for a collection of streamtraces for cylinders with aspect ratio 9 and 3 respectively.

thermal profile's periodicity and increase the reagents' exposure to intermediate temperatures. Thus, Poincaré maps can be used to visualize the extent of mixing present in a flow. 


\section{CORRELATION DIMENSION}

A quantitative approach to labeling a flow field as chaotic is through the use of correlation dimensions. Grassberger and Procaccia [34] introduced a methodology for computing the correlation dimension and this method has been extensively used in literature to characterize chaotic attractors. The advantage with using this method is that one can compute the chaotic nature of the flow field from a one-dimensional time series data which represents the flow field. Thus, nonlinear time-series and chaos analysis techniques can be used to gain insight and observe the dynamical nature of the flow field in these systems. The methodology followed for computing the correlation dimension in the current work is described in [35] and [36]. This technique is briefly described here.

The correlation sum $C^{d}(r)$ for a one dimensional time series data given by $x=\left\{x_{1}, x_{2}\right.$, $\left.x_{3}, \ldots, x_{\mathrm{n}}\right\}$ is given by Eq.(16):

$$
C^{d}(r)=\frac{1}{n(n-1)} \sum_{i=1}^{n} \sum_{j=1, j \neq i}^{n} \Theta\left(r-\left|\vec{x}_{i}-\vec{x}_{j}\right|\right)
$$

where, $\Theta$ is the Heaviside step function given by Eq.(17):

$$
\begin{array}{ll}
\Theta(x)=0 & \text { if } x<0 \\
\Theta(x)=1 & \text { if } x>0
\end{array}
$$

The superscript for the variable $C$ in the left-hand side of Eq.(16), given by $d$ indicates the number of embedding dimensions, which is used to define a $d$-dimensional vector with $d$ components, given by Eq.(18):

$$
\vec{x}_{i}=\left(x_{i}, x_{i+1}, x_{i+2}, \ldots, x_{i+(d-1)}\right)
$$

The term $\left|\vec{x}_{i}-\vec{x}_{j}\right|$ is defined using Eq.(19) 


$$
\left|\vec{x}_{i}-\vec{x}_{j}\right|=\sqrt{\sum_{k=0}^{d-1}\left(x_{i+k}-x_{j+k}\right)^{2}}
$$

The correlation dimension $D^{(d)}$ is then defined by Eq.(20), for some values of $r$, determined by the "scaling region":

$$
C^{(d)}(r) \propto r^{-D(d)}
$$

It is easy to spot the scaling region in certain scenarios by plotting $\log \left(C^{(d)}\right)$ versus $\log (r)$, this is true when the data shows a simplistic behavior which is readily apparent in case of a sine function or a random data set. However, in case of data sets where the scaling region is not easily distinguished, it is possible to identify it by plotting $\log \left(C^{(d)}(r)\right) / \log (r)$ - one would then observe a flat plateau region, which is an indication of the scaling region, where the slope of $\log \left(C^{(d)}(r)\right) / \log (r)$ is relatively stable [36]. In order to compute the actual correlation dimension, $D$ is computed for $\mathrm{d}=1,2,3 \ldots$ and these values are plotted as a function of $d$. It will be seen that when $d$ reaches a saturation value $\left(d_{\text {sat }}\right)$, the correlation dimension becomes independent of the embedding dimension $d$. This saturated value of $D$ gives us the multi-dimensional correlation dimension.

Based on the value of the correlation dimension $D$, one can predict the nature of the flow field using the definitions proposed by [37]. It can be readily understood that for higher dimensional systems, there will be more ways for points to be closer together, thus the number of pairs of points that lie close to each other will rise more rapidly for higher dimensions. For example, in the case of random numbers, as the size of the embedding dimension increases, the correlation sum increases, because the nature of 
random numbers makes it possible that there will more points lying in the vicinity of a given point, thus one can find a number of pairs of points that lie within a given distance. Thus, the correlation dimension can be expected to increase as the embedding dimension increases. In contrast, considering the case of a periodic function, like a sine function, as the embedding dimension increases, for a constant value of $r$, there is a limit to the number of pairs of points that lie within a distance of $r$ from each other. Increasing the embedding dimension beyond a certain value will result in a constant value of the correlation dimension. It has been established in literature that if, as d grows, $D$ tends to a value of 1 , then the time series is periodic. If $D$ tends to a value of 2 , then the time series is quasi-periodic. If $D$ tends to a constant value, greater than 2 , then the time series is chaotic and finally if $D$ continues to increase as d increases, then the time series is random. [37].

In order to validate our algorithm, we applied the procedure described above on time series data obtained from a periodic and a random signal, as shown below:

1. Periodic function: $x=\sin (t)$

2. Random function: Gaussian function distributed between 0 and 1 .

The data sets for the periodic and random functions proved to be simplistic, so that the scaling region was easily identified by plotting $\log \left(C^{(d)}\right)$ versus $\log (r)$. The correlations dimension was computed until $d=15$ (when the values converged within an accuracy of $2 \%$ ) for the periodic and random function. These plots are shown in Fig. 28. Using the algorithm described above, the values of correlation dimension obtained for the periodic function was 1 , which is as expected. In case of the random variables, the value of the 
(a)

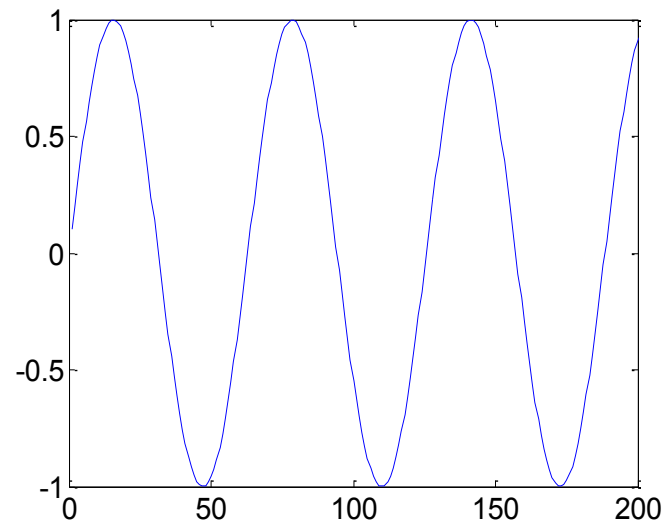

(c)

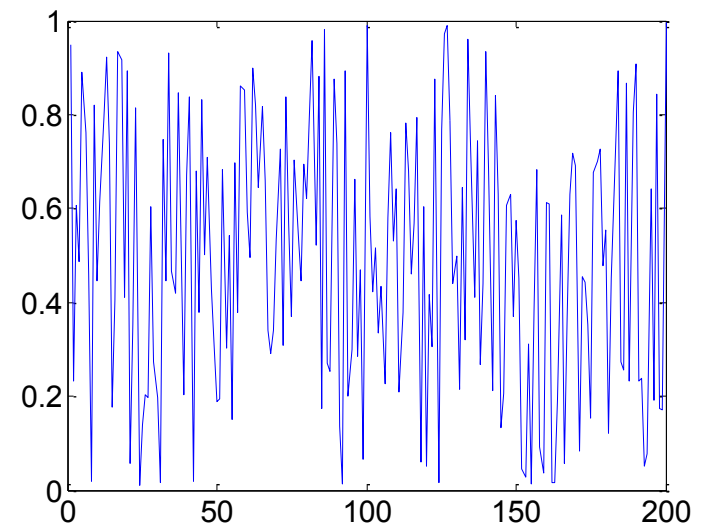

(e)

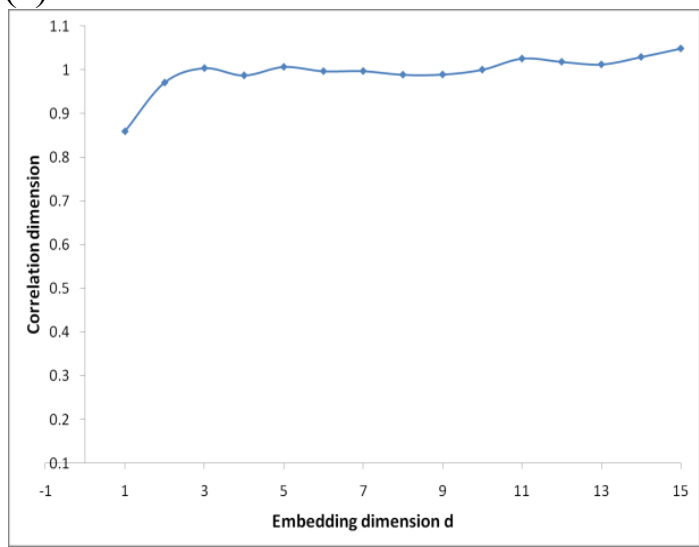

(b)

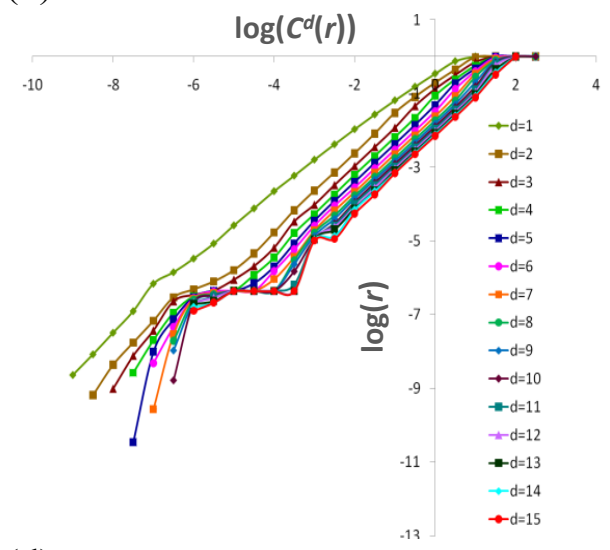

(d)

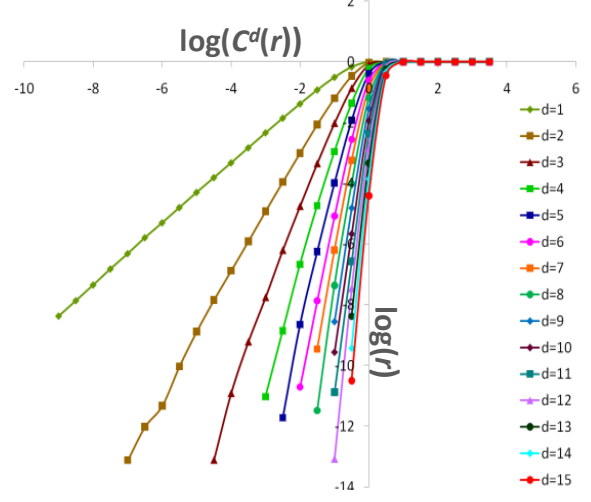

(f)

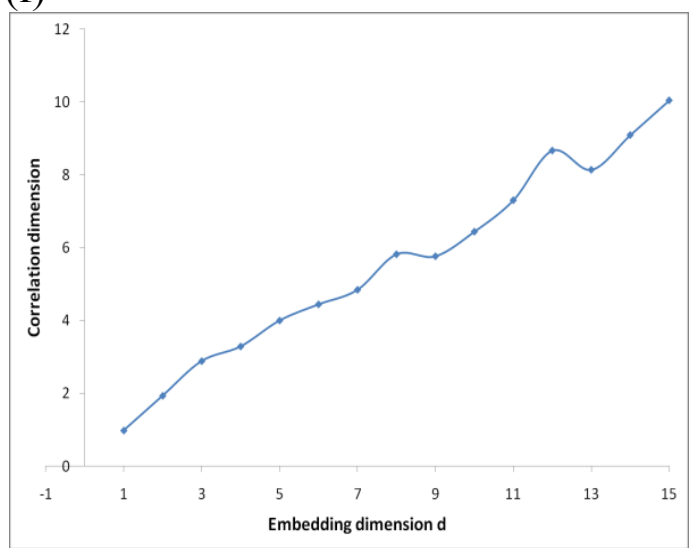

Fig. 28 (a) and c). Plots showing the sine function and the random function respectively. Fig. 28 (b) and (d). Plots showing the log-log plot of the correlation sum versus the distance $r$ for the sine and random functions respectively. Fig. 28(e) and (f). Plots showing the variation of the correlation dimension versus the embedding dimension for the sine and random functions. 
correlation dimension increases as the embedding dimension increases, this is also an expected result. Therefore, we can be confident in the algorithm to compute the values of the correlation dimension for the time series data obtained from the Rayleigh-Bénard system. The number of data points was chosen to be 30000 , values of $r$ ranged from $10^{-6}$ through 10 and the calculation for the correlation dimension was performed for $d=1$ through $d=15$, until the solution converged within an accuracy of $3 \%$.

Fig. 29(a) shows the plot of the correlation integral versus $r$ for the cylinder with aspect ratio 3 and volume $30 \mu \mathrm{l}$ - one can observe that there is a scaling region for certain values of $r$, however, it is difficult to narrow down the region with accuracy. In these cases, it was necessary to plot $\log \left(C^{(d)}(r)\right) / \log (r)$, as shown in Fig. 29(b), which shows a plateau region for certain values of $r$. This region indicates the scaling region and the value of the correlation dimension can be calculated for the points that lie in this region. The values of the correlation dimension obtained for $h / d=3$ was 2.64 using this method (Fig. 29(e)). The same method was followed for the cylinder with aspect ratio 9 (Fig. 29(c), 29(d) and 29(f)) and the computed value of the correlation dimension was 2.08. This provides further proof that while the cylinder with aspect ratio 9 provided a quasiperiodic flow, the flow field in the cylinder with aspect ratio 3 is indeed chaotic.

Fig. 30 shows the spectrum of correlation dimensions for aspect ratios ranging from 0.5 to 9 for cylinders of volume 10,30 and $50 \mu$. This enables the "strength" of the chaotic component of the flow to be quantified, so that convective flow fields with a prescribed chaotic component can be rationally selected. The temperature plots corresponding to each of the above cases is shown in Fig. 31, 32 and 33. 
(a)

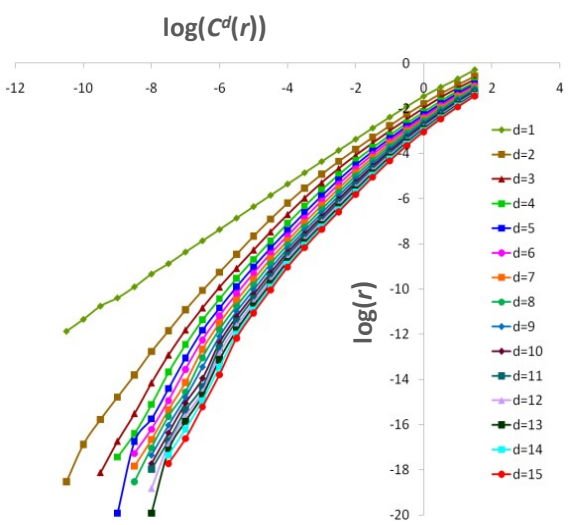

(c)

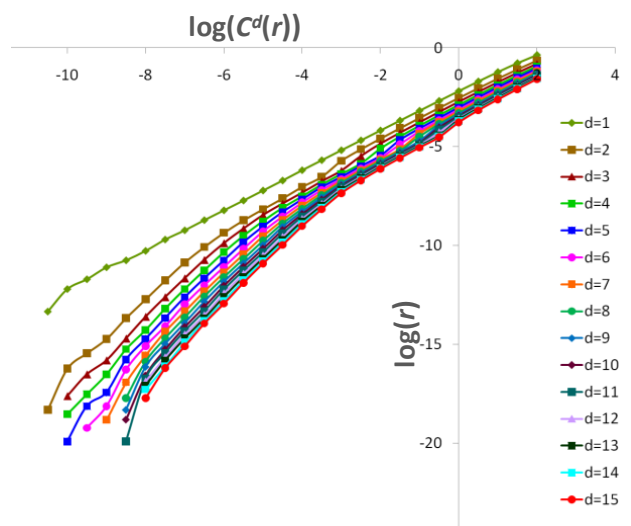

(e)

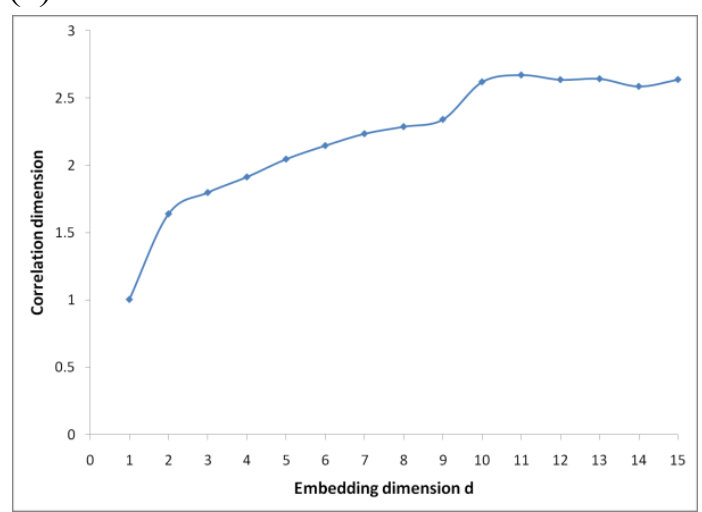

(b)

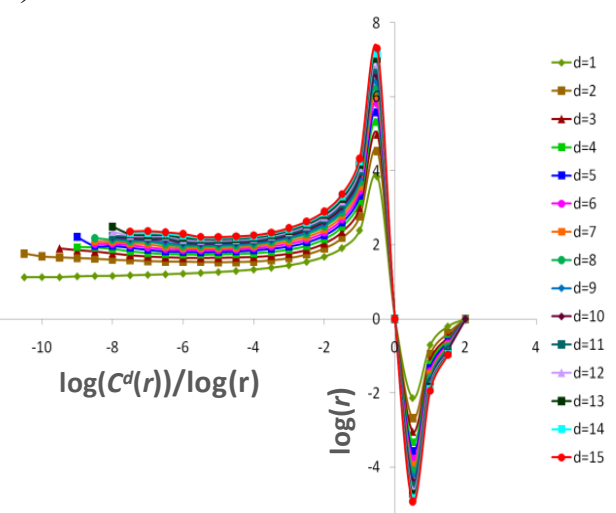

(d)

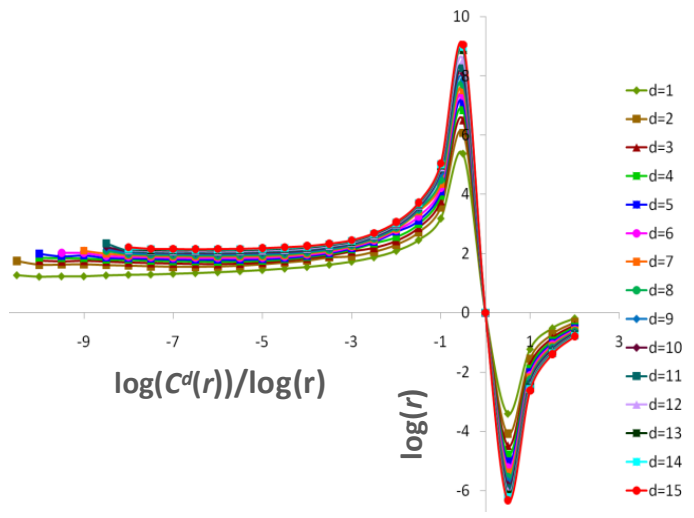

(f)

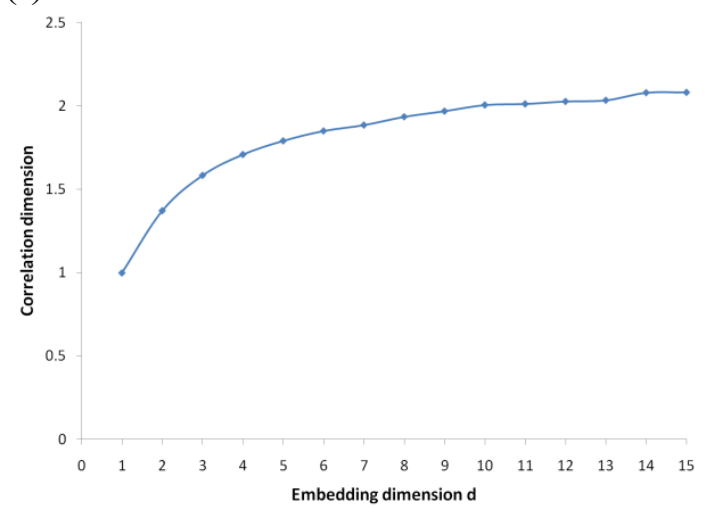

Fig. 29 (a) and (c) Plots showing the log-log plot of the correlation sum versus the distance $r$ for the cylinders with aspect ratio 3 and 9 respectively. Fig. 29 (b) and (d) Plots showing the plot of $\log \left(\mathrm{C}^{(\mathrm{d})}(\mathrm{r})\right) / \log (\mathrm{r})$ versus $\log (\mathrm{r})$. Fig. 29(e) and (f) Plots showing the variation of the correlation dimension versus the embedding dimension for the cylinders with aspect ratio 3 and 9 respectively. 


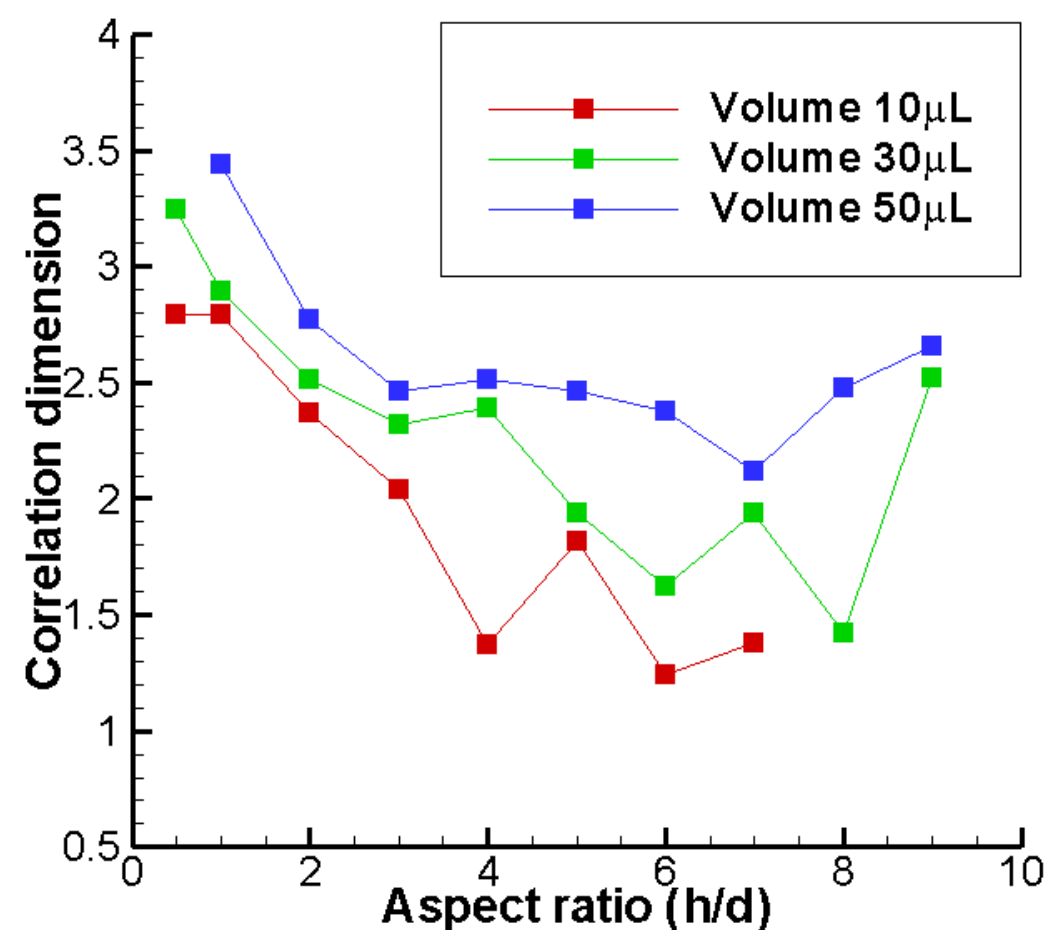

Fig. 30 Correlation dimensions for cylinders of varying volumes and aspect ratios.

(a) Volume $=10 \mu \mathrm{L}, h / d=0.5$

(b) Volume $=10 \mu \mathrm{L}, h / d=1$

(c) Volume $=10 \mu \mathrm{L}, h / d=2$
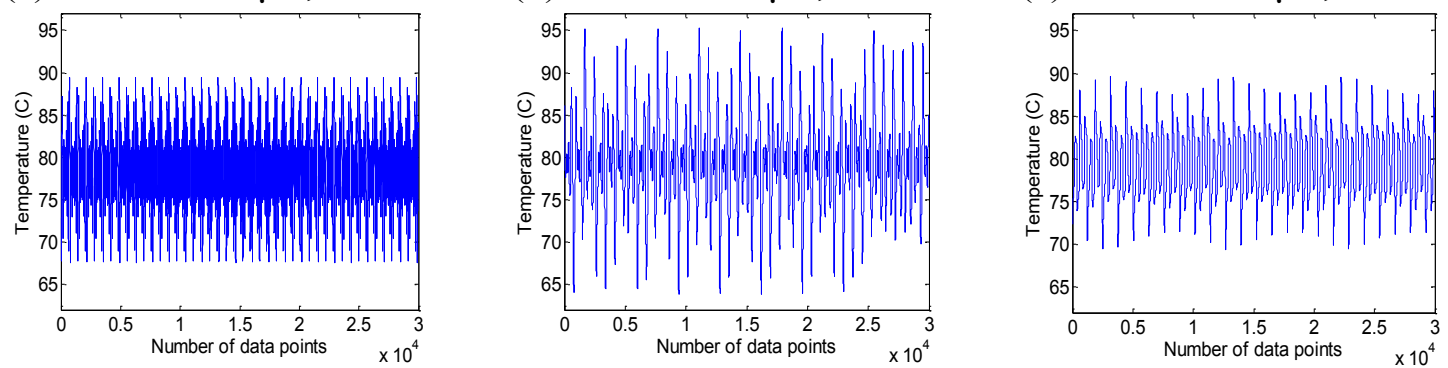

Figs. 31 Temperature contours for $10 \mu \mathrm{L}$ cylinders and different aspect ratios. 
(a) Volume $=10 \mu \mathrm{L}, h / d=3$

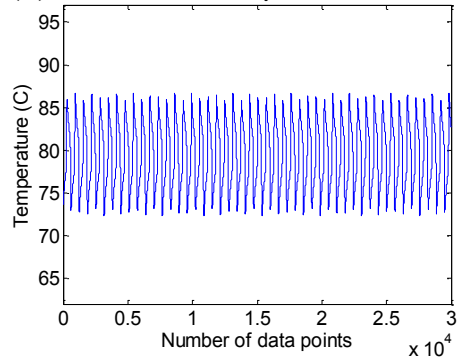

(d) Volume $=10 \mu \mathrm{L}, h / d=6$

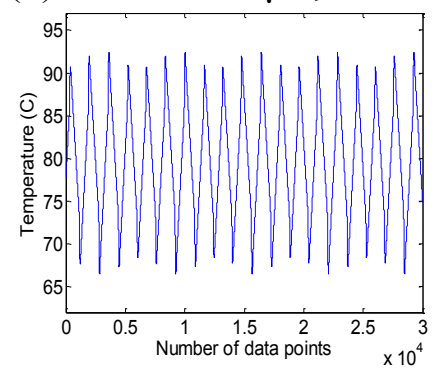

(g) Volume $=30 \mu \mathrm{L}, h / d=1$

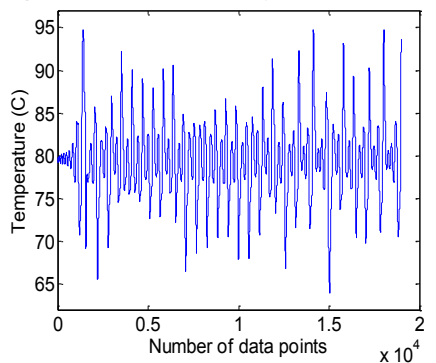

(j) Volume $=30 \mu \mathrm{L}, h / d=4$

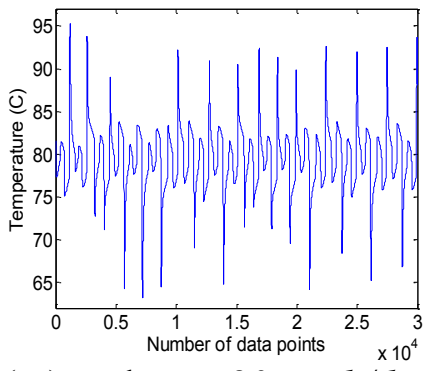

(m) Volume $=30 \mu \mathrm{L}, h / d=7$

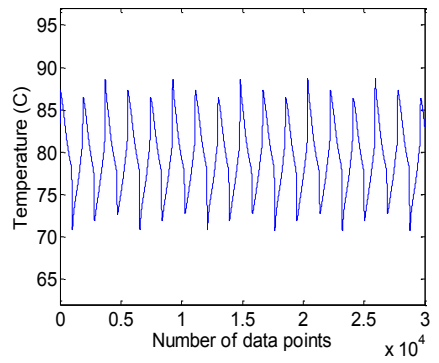

(b) Volume $=10 \mu \mathrm{L}, h / d=4$

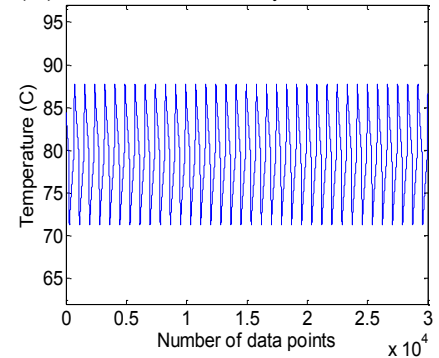

(e) Volume $=10 \mu \mathrm{L}, h / d=7$

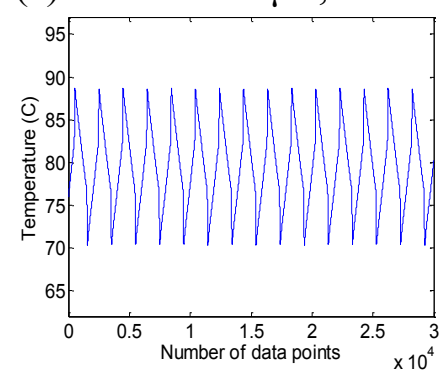

(h) Volume $=30 \mu \mathrm{L}, h / d=2$

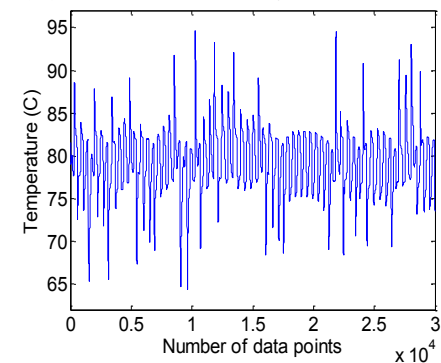

(k) Volume $=30 \mu \mathrm{L}, h / d=5$

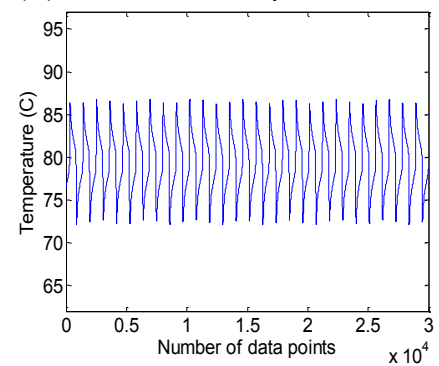

(n) Volume $=30 \mu \mathrm{L}, h / d=8$

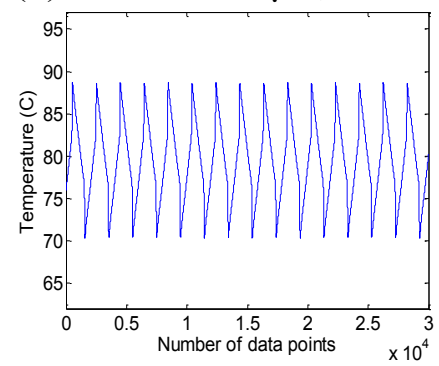

(c) Volume $=10 \mu \mathrm{L}, h / d=5$

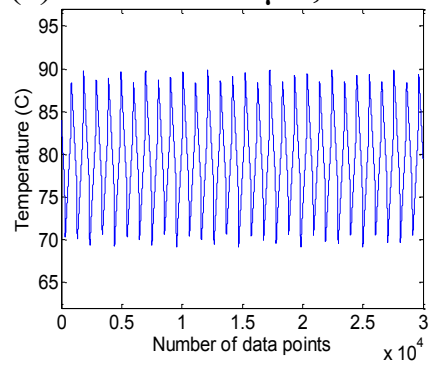

(f) Volume $=30 \mu \mathrm{L}, h / d=0.5$

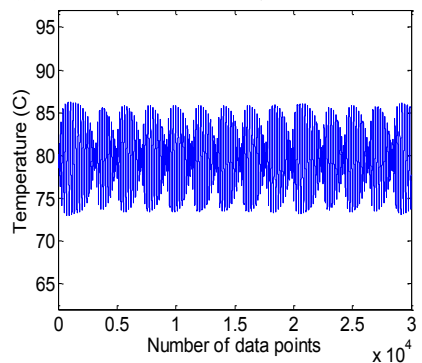

(i) Volume $=30 \mu \mathrm{L}, h / d=3$

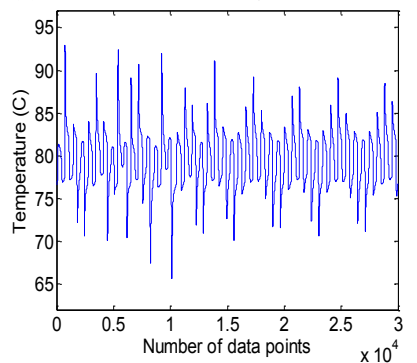

(1) Volume $=30 \mu \mathrm{L}, h / d=6$

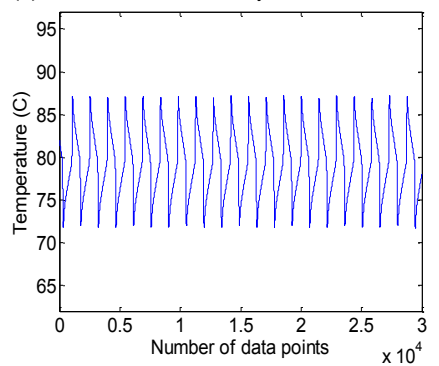

(o) Volume $=30 \mu \mathrm{L}, h / d=9$

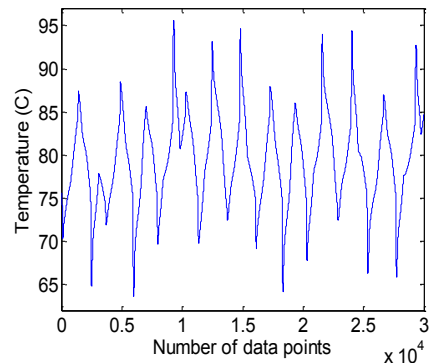

Figs. 32 Temperature contours for cylinders of varying volumes and aspect ratios. 
(a) Volume $=50 \mu \mathrm{L}, h / d=1$

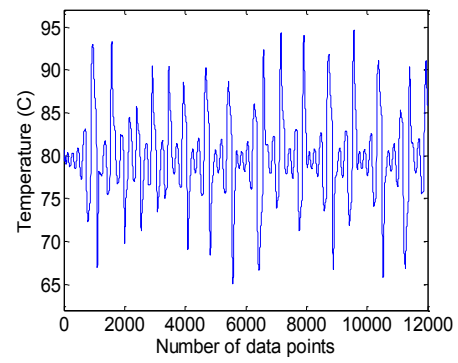

(d) Volume $=50 \mu \mathrm{L}, h / d=4$

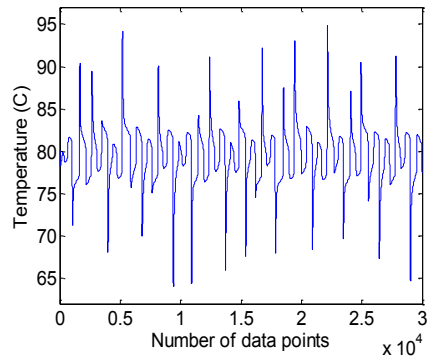

(g) Volume $=50 \mu \mathrm{L}, h / d=7$

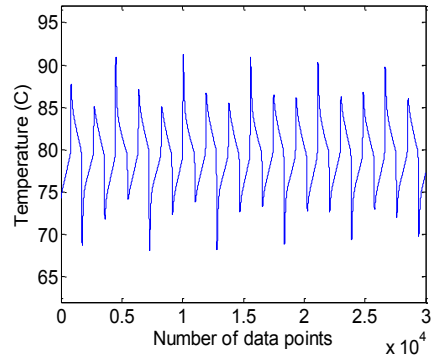

(b) Volume $=50 \mu \mathrm{L}, h / d=2$

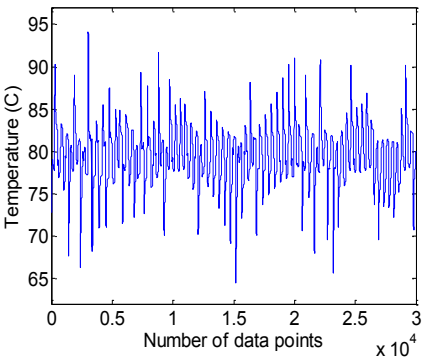

(e) Volume $=50 \mu \mathrm{L}, h / d=5$

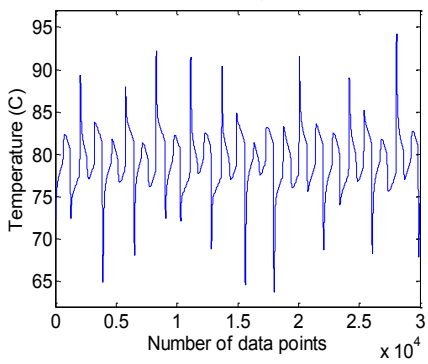

(h) Volume $=50 \mu \mathrm{L}, h / d=8$

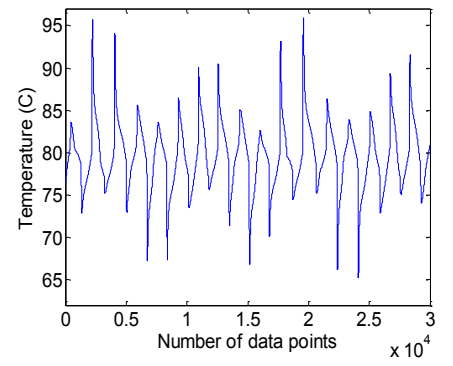

(c) Volume $=50 \mu \mathrm{L}, h / d=3$

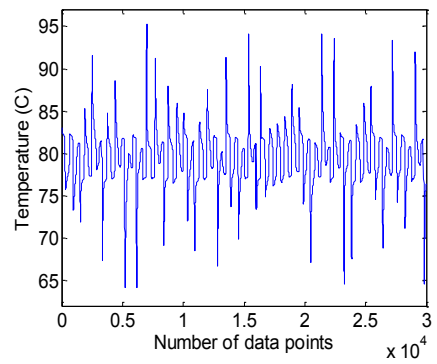

(f) Volume $=50 \mu \mathrm{L}, h / d=6$

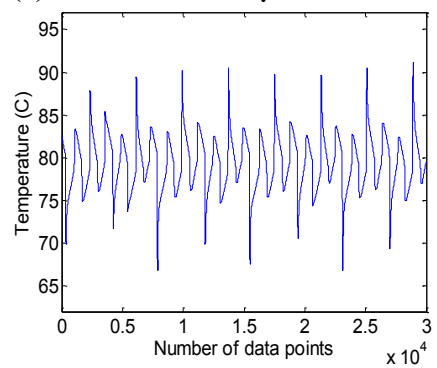

(i) Volume $=50 \mu \mathrm{L}, h / d=9$

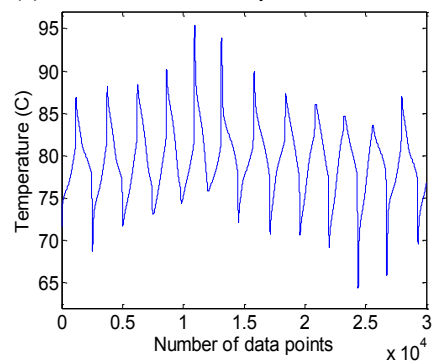

Figs. 33 Temperature contours for cylinders of volume $50 \mu \mathrm{L}$ and aspect ratios.

\section{TRANSITIONS BETWEEN FLOWS}

Although chaotic advection has been previously explored in the Rayleigh-Bénard system, past studies have focused exclusively on geometries at $h / d<1$ involving an oscillatory horizontal perturbation superimposed over the $2 \mathrm{D}$ convective base flow that introduces a time-periodic component to the fluid motion (i.e., the "even" oscillatory instability) [38-40]. In contrast, 3D chaotic flows have received considerably less attention, largely owing to the lack of convenient model systems in which to study them 
[41]. Rayleigh-Bénard convection at $h / d>1$ fulfills this need by providing a robust platform to probe a wide variety of 3D flows incorporating features ranging from quasiperiodic motion to full blown chaos [42]. To highlight the spectrum of accessible flow states, we performed simulations under conditions where three different temperature gradients were applied between the top and bottom surfaces of $30 \mu \mathrm{L}$ reactors at $h / d=3$ $\left(\triangle T=5,35\right.$, and $\left.100^{\circ} \mathrm{C}\right)$ and $h / d=8\left(\triangle T=35,50\right.$, and $\left.200^{\circ} \mathrm{C}\right)$ (Fig. 34).

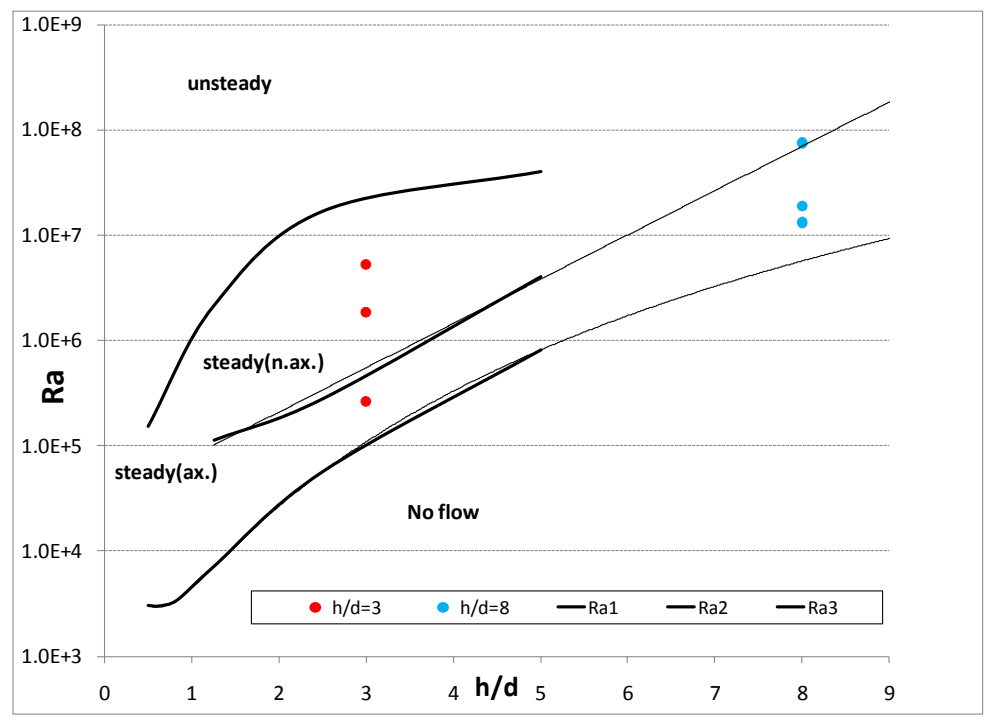

Fig. 34 Schematic of points used for constant aspect ratios 3 and 8, for constant volume 30ul and varying Rayleigh numbers.

Decreasing $\triangle T$ from PCR conditions $\left(\triangle T=35^{\circ} \mathrm{C}\right)$ to $5{ }^{\circ} \mathrm{C}$ at $h / d=3$ induces a transition from chaotic trajectories in Fig. 35 to a 2D circulatory profile with quasiperiodic characteristics evident in the emergence of KAM boundaries in the Poincaré map. Conversely, increasing $\triangle T$ from 35 to $100{ }^{\circ} \mathrm{C}$ causes the flow pattern to become 
even more disordered, accompanied by signatures of multiple convection loop structures in qualitative agreement with the flow regime boundaries previously identified at $h / d<5$ [5].

(a)

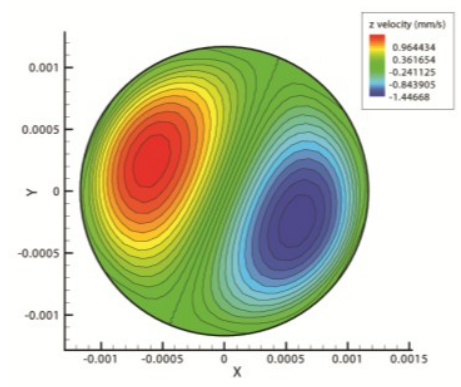

(d)

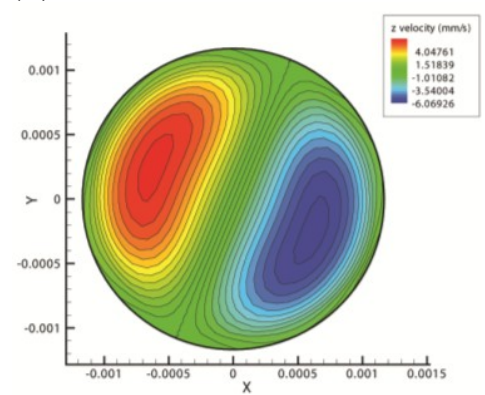

(g)

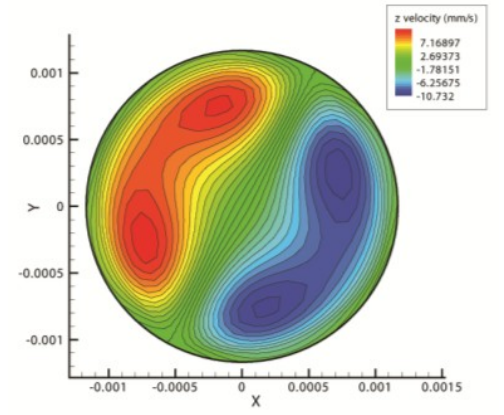

(b)

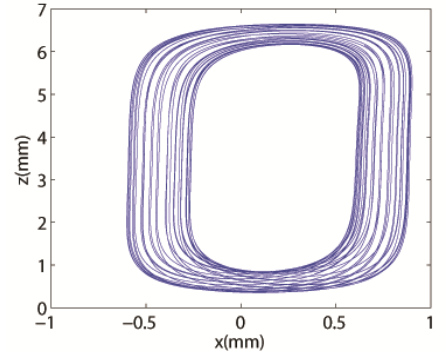

(e)

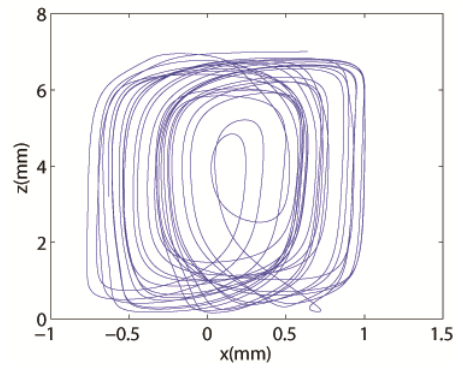

(h)

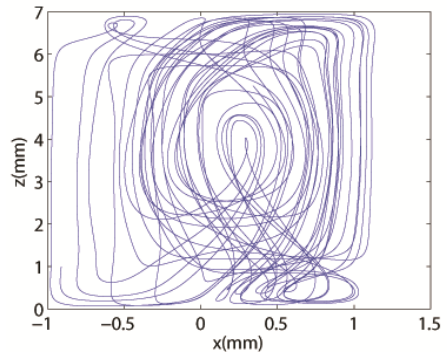

(c)

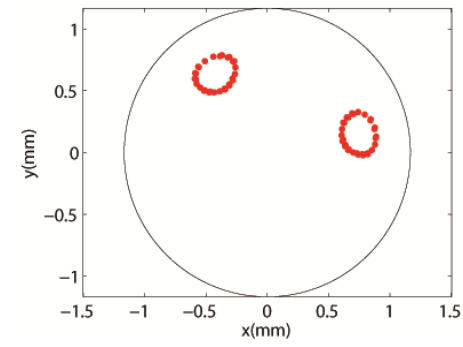

(f)

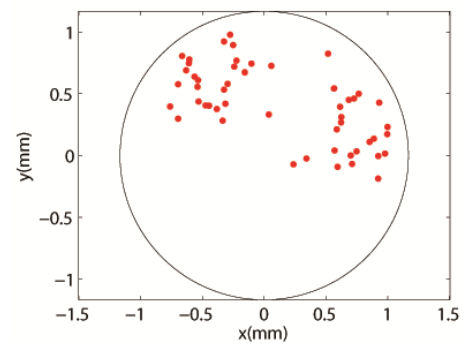

(i)

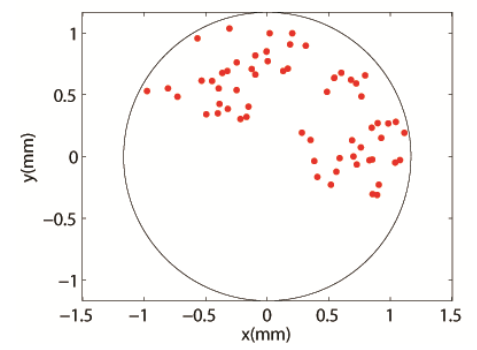

Fig. 35(a)-(c) Plots of the $\mathrm{z}$ velocity contours, streamtraces and Poincaré sections for cylinder with aspect ratio 3 and $\Delta \mathrm{T}=5^{\circ} \mathrm{C}$. Fig. 35(d)-(f) Plots of the z velocity contours, streamtraces and Poincare sections for cylinder with aspect ratio 3 and $\Delta \mathrm{T}=35^{\circ} \mathrm{C}$. Fig. 35(g)-(i) Plots of the $\mathrm{z}$ velocity contours, streamtraces and Poincaré sections for cylinder with aspect ratio 3 and $\Delta \mathrm{T}=100^{\circ} \mathrm{C}$. 
A similar cascade is evident at $\mathrm{h} / \mathrm{d}=8$, where the closed-loop quasi-periodic motion under PCR conditions $\left(\triangle T=35^{\circ} \mathrm{C}\right.$ ) gives way to chaotic advection as $\Delta T$ is increased to $200{ }^{\circ} \mathrm{C}$, shown in Fig. 36. The same kind of transition is also initiated by moving from $h / d=8$ to 3 at $\Delta T=35^{\circ} \mathrm{C}$. These results further support the capability to

(a)

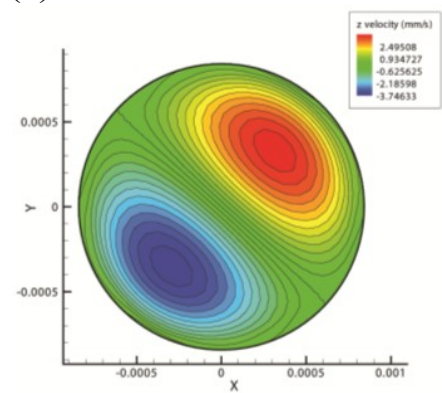

(d)

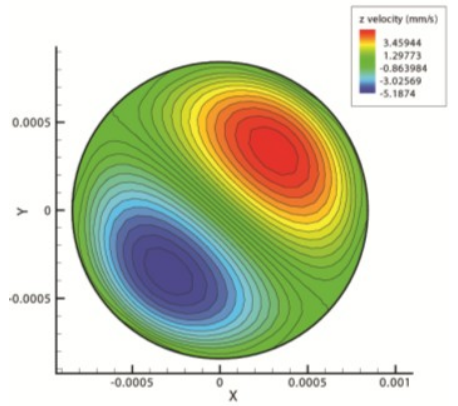

(g)

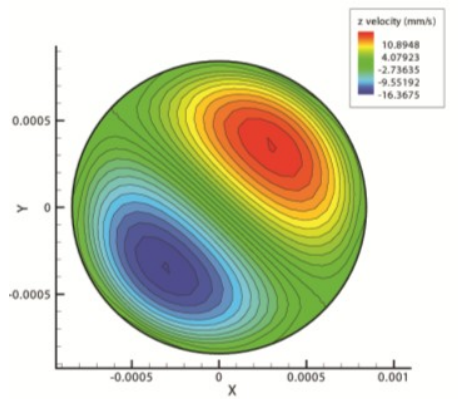

(b)

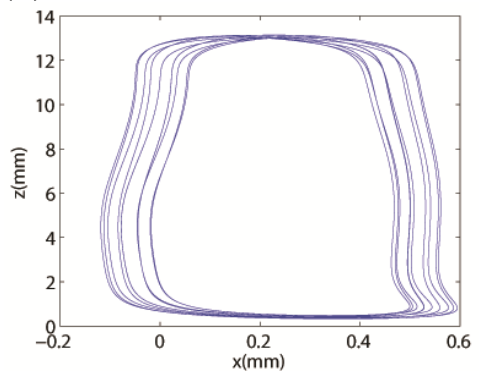

(e)

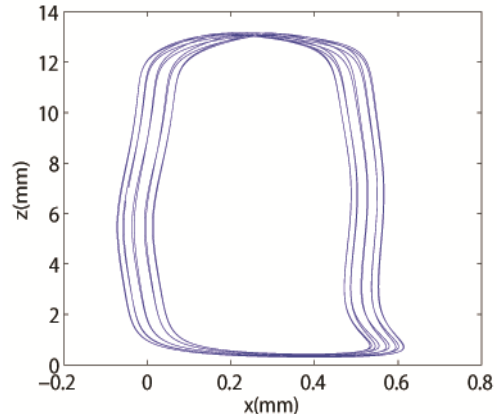

(h)

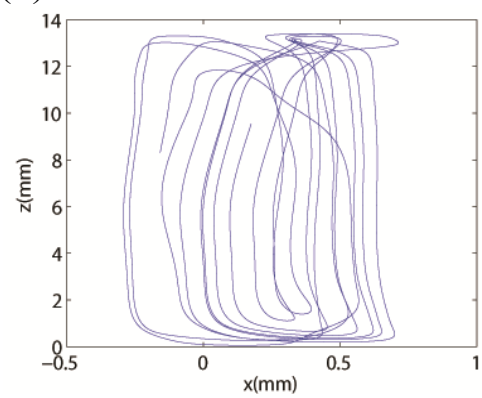

(c)

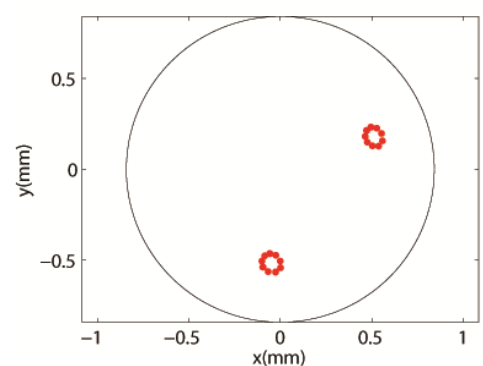

(f)

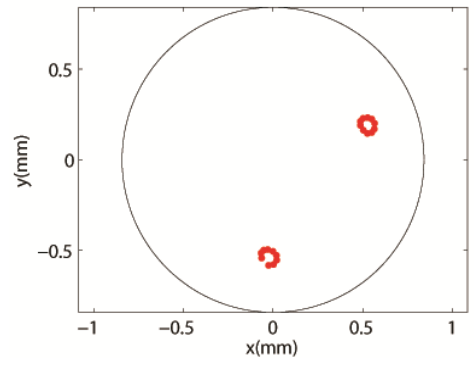

(i)

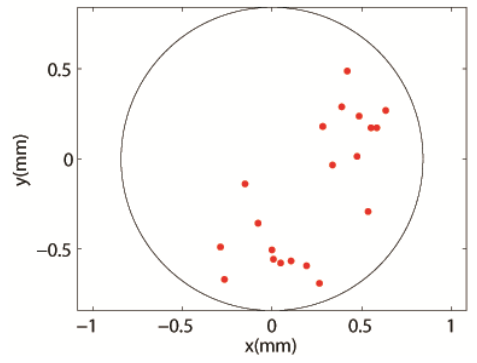

Fig. 36(a)-(c) Plots of the $\mathrm{z}$ velocity contours, streamtraces and Poincaré sections for cylinder with aspect ratio 8 and $\Delta \mathrm{T}=35^{\circ} \mathrm{C}$. Fig. 36(d)-(f) Plots of the $\mathrm{z}$ velocity contours, streamtraces and Poincaré sections for cylinder with aspect ratio 8 and $\Delta \mathrm{T}=50$ ${ }^{\circ}$ C. Fig. 36(g)-(i) Plots of the $\mathrm{z}$ velocity contours, streamtraces and Poincaré sections for cylinder with aspect ratio 8 and $\Delta \mathrm{T}=200{ }^{\circ} \mathrm{C}$. 
select from a broad range of flow states in order to introduce a prescribed chaotic component to the fluid motion. Another study was performed for cylinders of volume 30 $\mu \mathrm{L}$, with aspect ratios varying between 0.5 and $9, R a=10^{7}$, with the bottom surface always maintained at $370 \mathrm{~K}$, for the sake of generality. It was found that under the current parameter range, the cases with aspect ratio $0.5,1$ and 2 resulted in the temperature of the top surface being less than zero, these cases were therefore discarded. Thus, simulations were performed only for cylinders whose aspect ratio falls between 3 and 9. A schematic of the chosen points is shown in Fig. 37.

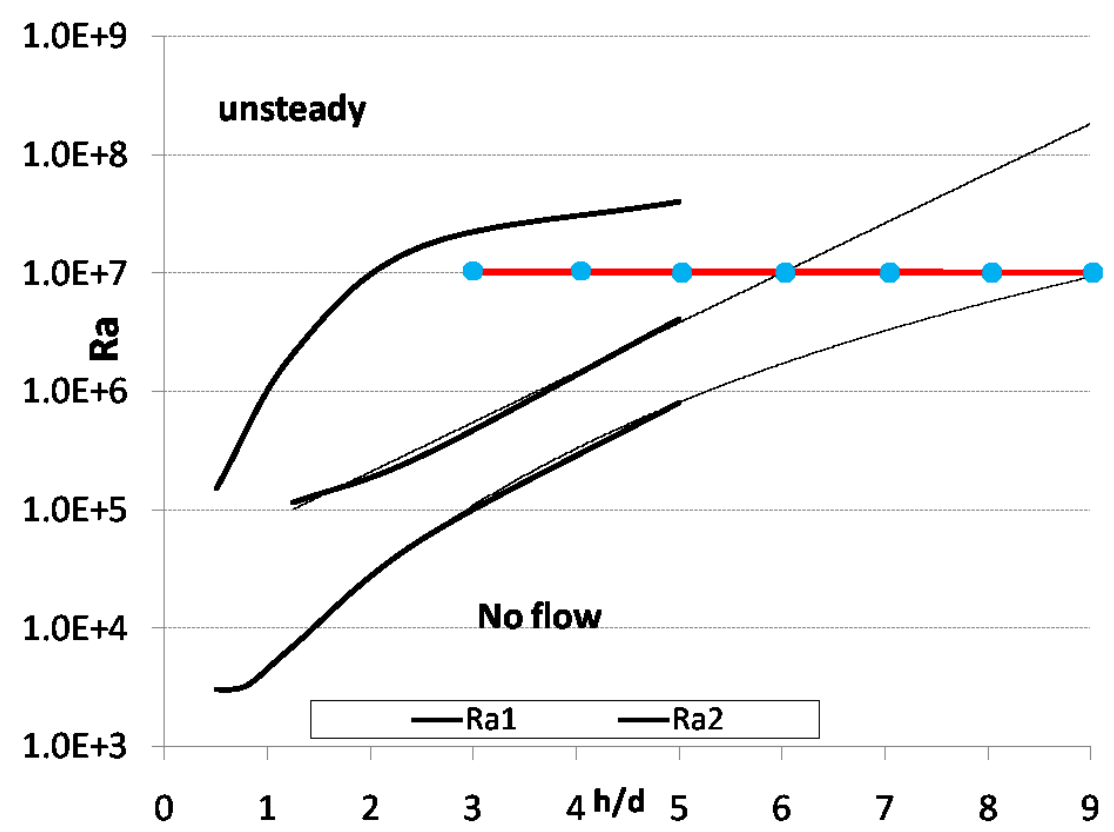

Fig. 37 Schematic of points used for cylinders of constant volume 30ul and constant Rayleigh number $=10^{7}$ and varying aspect ratios. 
Starting with the cylinder of aspect ratio 9 , with $\Delta \mathrm{T}=21^{\circ} \mathrm{C}$, we found that we obtained a conduction solution, which is expected from Fig. 37 since it lies at the transition between the no flow case and the start of natural convection. Continuing along with the cylinder of aspect ratio 8 , with $\Delta \mathrm{T}=27{ }^{\circ} \mathrm{C}$, we found that we obtained a convection solution whose behavior was similar to that obtained from the PCR conditions i.e. the fluid motion was characterized by quasiperioidic flow between the upper and lower extremes, and the Poincaré maps showed evidence of the close-knit behavior of the streamtrace. Similar behavior was observed with the cylinder of aspect ratio 7 and $\Delta \mathrm{T}=35^{\circ} \mathrm{C}$.

(a)

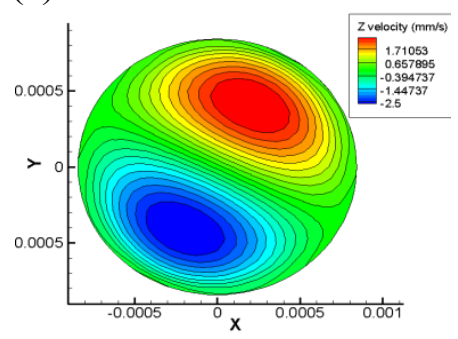

(d)

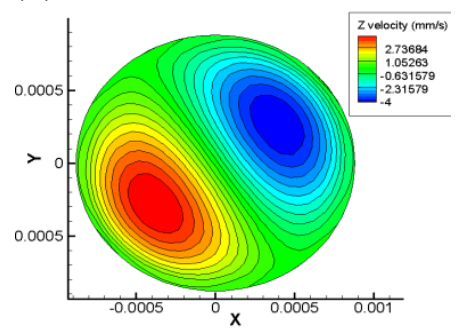

(b)

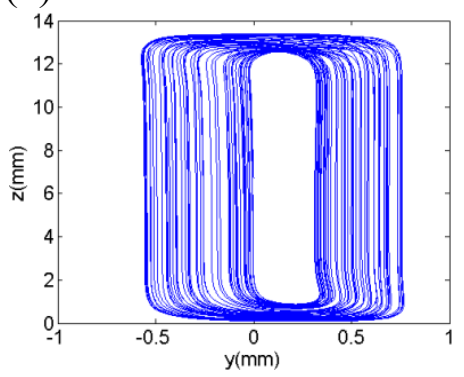

(e)

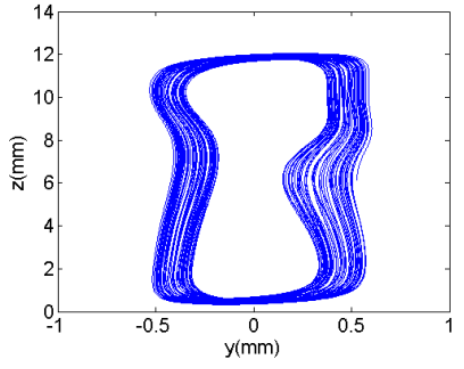

(c)

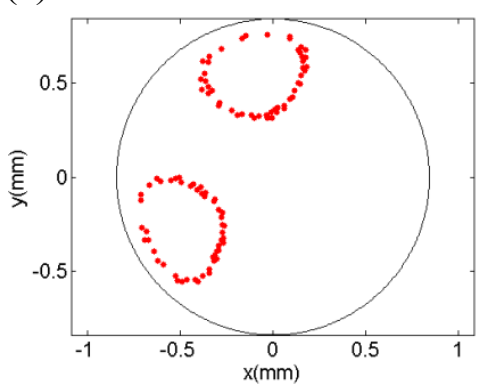

(f)

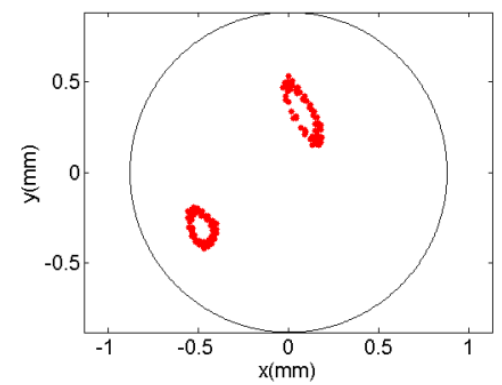

Fig. 38(a)-(c) Plots of the $\mathrm{z}$ velocity contours, streamtraces and Poincare sections for cylinder with aspect ratio 8 and $\Delta \mathrm{T}=27^{\circ} \mathrm{C}$. Fig. 38(d)-(f) Plots of the $\mathrm{z}$ velocity contours, streamtraces and Poincaré sections for cylinder with aspect ratio 7 and $\Delta \mathrm{T}=35$ ${ }^{\circ} \mathrm{C}$. 
Moving onto the cylinder of aspect ratio 6 , with $\Delta \mathrm{T}=47^{\circ} \mathrm{C}$, we can begin to see the disordered nature of the flow - the Poincaré section shows evidence of the streamtraces starting to spread out. This is even more evident in the cylinders with aspect ratio $5\left(\Delta \mathrm{T}=68{ }^{\circ} \mathrm{C}\right)$ and aspect ratio $4\left(\left(\Delta \mathrm{T}=106{ }^{\circ} \mathrm{C}\right)\right.$. Here, the streamtraces have spread out over the volume of the fluid, and the flow is highly chaotic. One can also notice that the maximum velocity of the field has been steadily increasing from $1.7 \mathrm{~mm} / \mathrm{s}$ in the case of the cylinder with aspect ratio 8 to $8.2 \mathrm{~mm} / \mathrm{s}$ in the case of the cylinder with aspect ratio 4. These simulations were modeled using a steady solver in FLUENT. Continuing along with simulations for aspect ratio 3, which lies closer to the critical Rayleigh number for unsteady flow, we observed that we were unable to obtain a converged solution using a steady state solver. We then used a second order implicit, unsteady solver in FLUENT and we found that we were able to obtain a converged solution for the flow - the time-changing velocity contours in the $\mathrm{xz}$ and yz planes are shown in Fig. 40 and 41. It is interesting to note the transitions that are possible in the cylinder with the aspect ratio 3 as time progresses at a very high Rayleigh number. The velocities are significantly higher than cases where the Rayleigh number was lower. The influence of aspect ratio at a constant Rayleigh number, for a fixed volume of fluid, was thus shown to be significant in the formation of patterns in the fluid flow. 
(a)

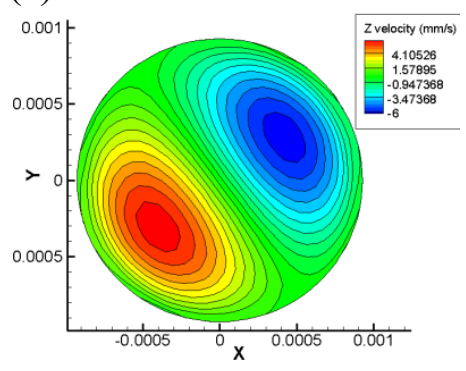

(d)

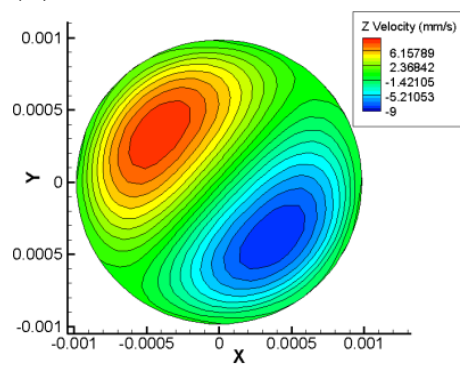

(g)

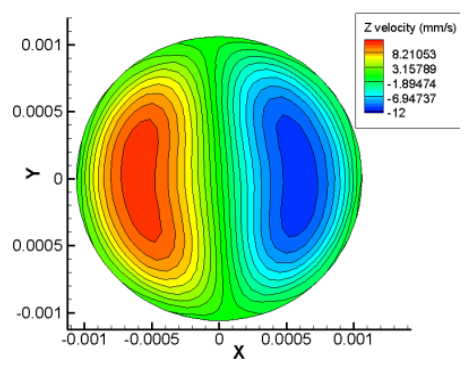

(b)

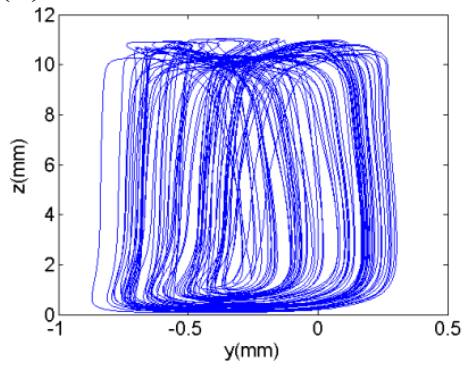

(e)

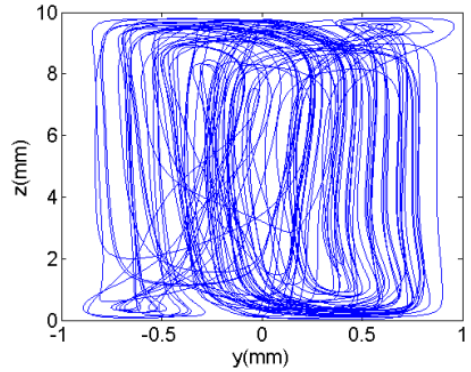

(h)

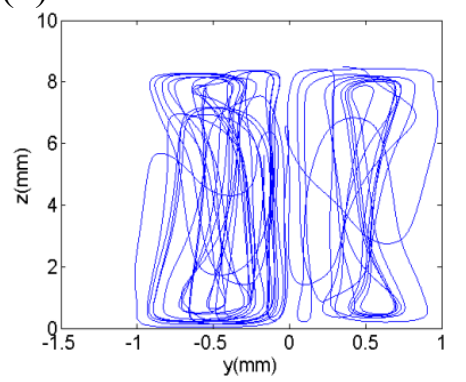

(c)

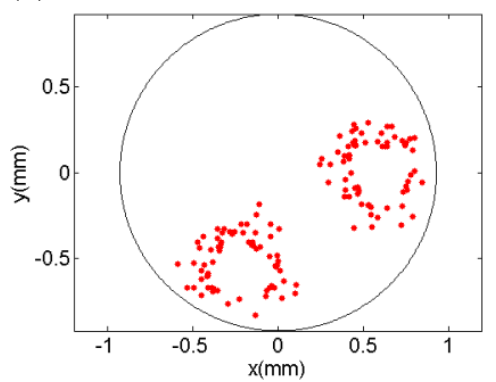

(f)

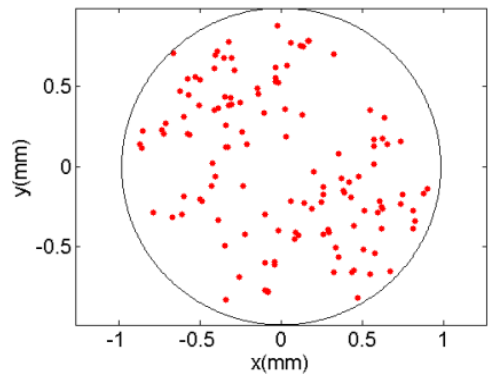

(i)

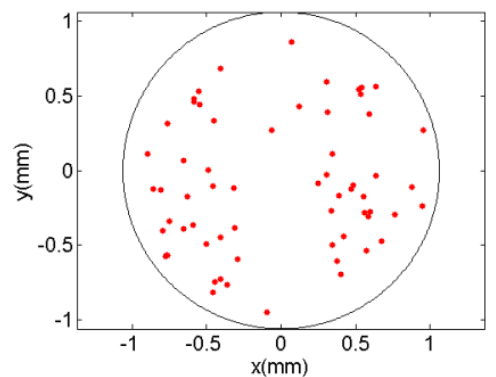

Fig. 39(a)-(c) Plots of the $\mathrm{z}$ velocity contours, streamtraces and Poincare sections for cylinder with aspect ratio 6 and $\Delta \mathrm{T}=47^{\circ} \mathrm{C}$. Fig. 39(d)-(f) Plots of the $\mathrm{z}$ velocity contours, streamtraces and Poincaré sections for cylinder with aspect ratio 5 and $\Delta \mathrm{T}=68$ ${ }^{\circ}$ C. Fig. 39(g)-(i) Plots of the z velocity contours, streamtraces and Poincaré sections for cylinder with aspect ratio 4 and $\Delta \mathrm{T}=106^{\circ} \mathrm{C}$. 
(a) $\mathrm{t}=0.11 \mathrm{~s}$

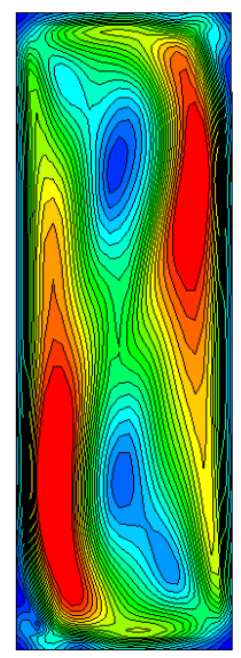

(e) t-19.91s

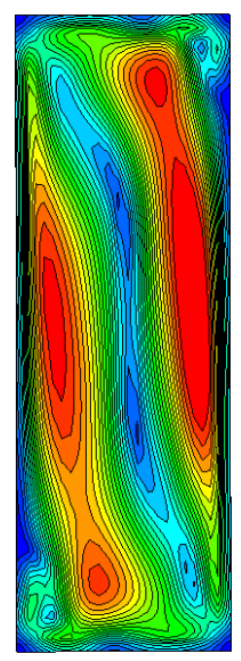

(b) $\mathrm{t}=4.91 \mathrm{~s}$

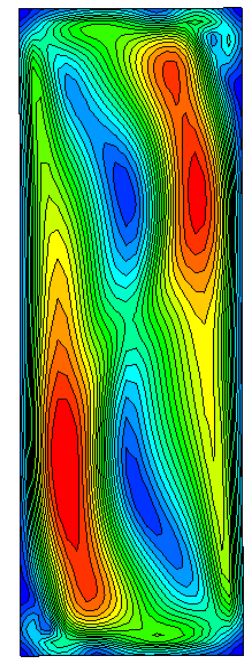

(f) $\mathrm{t}=24.91 \mathrm{~s}$

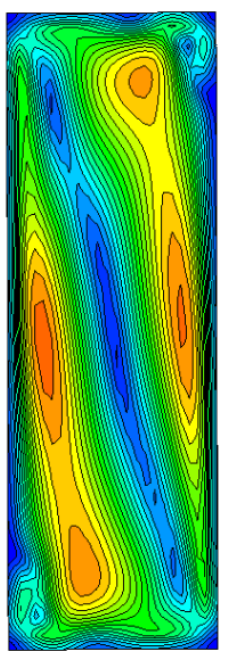

(c) $\mathrm{t}=9.91 \mathrm{~s}$

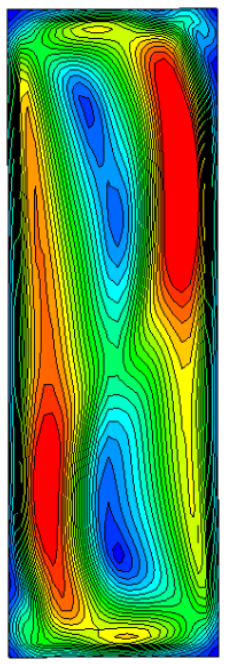

(g) $\mathrm{t}=29.91 \mathrm{~s}$

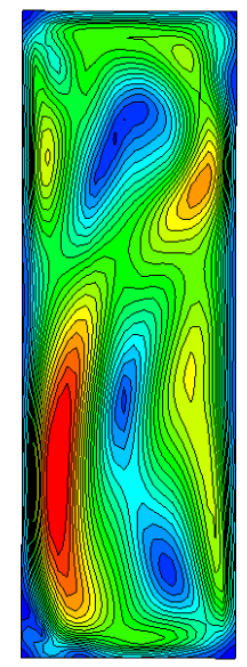

(d) $\mathrm{t}=14.91 \mathrm{~s}$

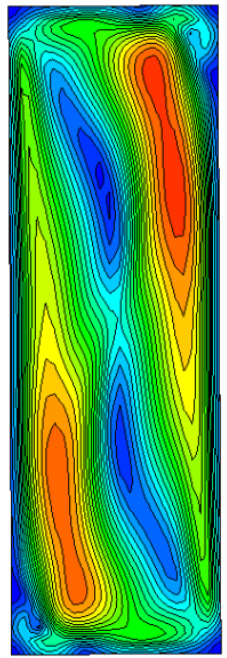

(h) $\mathrm{t}=32.91 \mathrm{~s}$

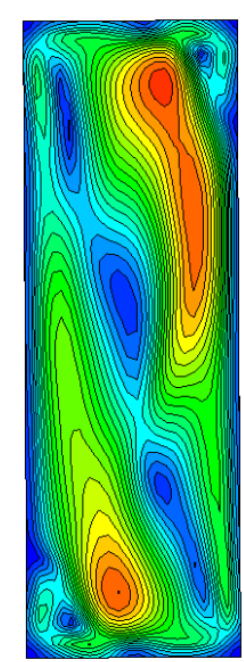

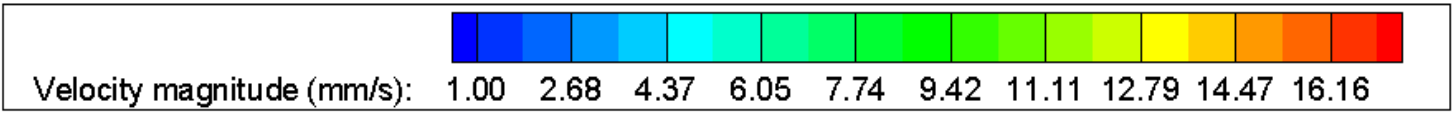

Fig. 40(a)-(h) Contour plots of velocity magnitude in the $\mathrm{XZ}$ plane for cylinder with aspect ratio 3 and $\Delta \mathrm{T}=189^{\circ} \mathrm{C}$, taken at different time instants. 
(a) $\mathrm{t}=0.11 \mathrm{~s}$

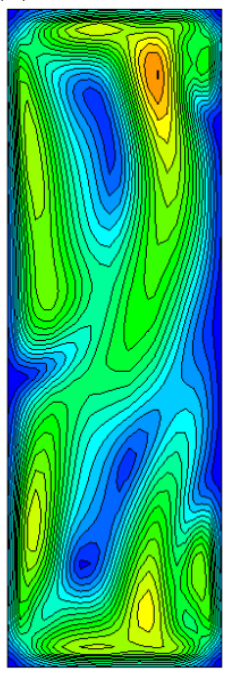

(e)t $=19.91 \mathrm{~s}$

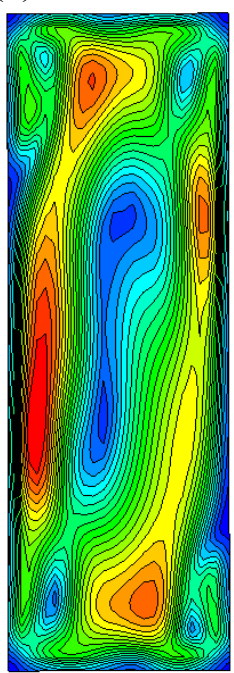

(b) $\mathrm{t}=4.91 \mathrm{~s}$

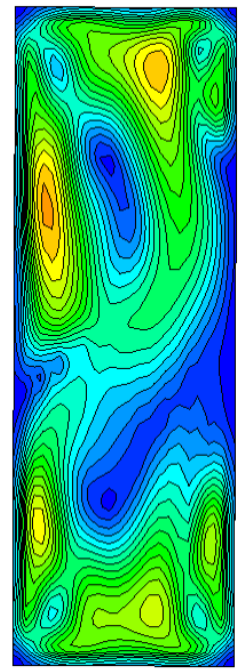

(f) $\mathrm{t}=24.91 \mathrm{~s}$

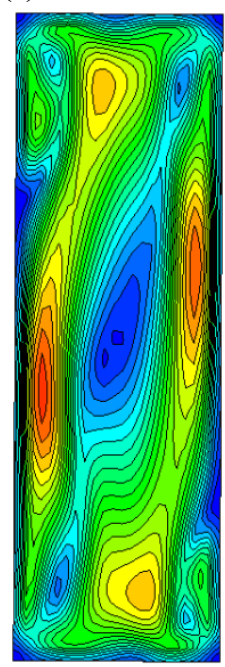

(c) $\mathrm{t}=9.91 \mathrm{~s}$

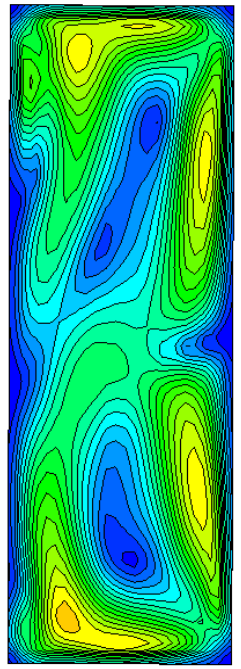

(g) $\mathrm{t}=29.91 \mathrm{~s}$

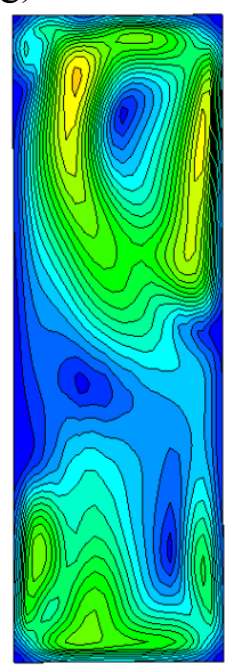

(d) $\mathrm{t}=14.91 \mathrm{~s}$

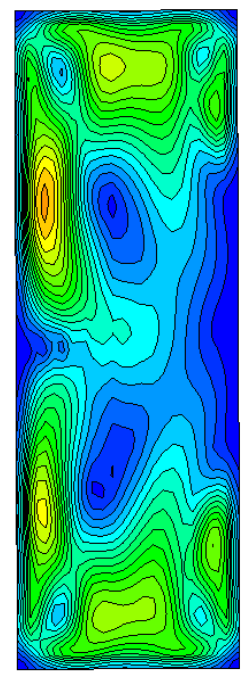

(h) $\mathrm{t}=32.91 \mathrm{~s}$

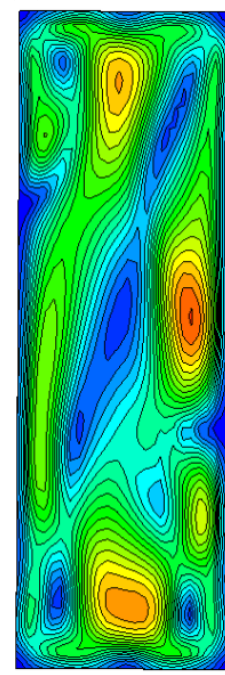

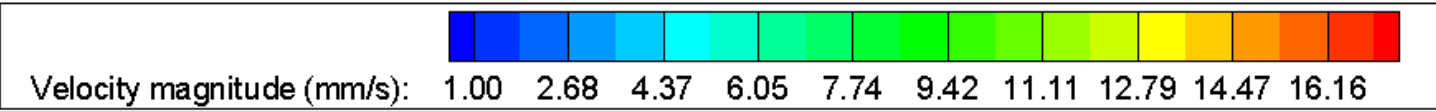

Fig. 41(a)-(h) Contour plots of velocity magnitude in the XZ plane for cylinder with aspect ratio 3 and $\Delta \mathrm{T}=189^{\circ} \mathrm{C}$, taken at different time instants. 


\section{CHAPTER V}

\section{FLOW MEASUREMENT TECHNIQUES}

\section{INTRODUCTION TO PARTICLE IMAGE VELOCIMETRY}

Particle image velocimetry is an optical, non-intrusive technique used for computing the velocity of a flow field. Here, the fluid is seeded with tracer particles whose size is very small so that the particles do not affect the flow field, and the velocity of the tracer particle can be assumed to be the same as that of the fluid flow. A planar sheet of laser light is used to illuminate the particles and a sequence of images are taken under short-pulse illumination [43]. The images can be analyzed based on a simple principle, that the velocity of the particle is obtained from the displacement of the particle divided by the time interval between the successive positions. This is further explained by Fig. 42.

The typical setup for PIV consists of a laser source, optics that guides the laser along its path to produce a planar sheet of laser required for illumination and a high speed camera that records the images. Modern day charge coupled device (CCD) cameras allow recordings of about 100 PIV images per min while high speed recordings on complementary metal-oxide semiconductor (CMOS) sensors allow recordings in the kilohertz range [44]. A schematic is shown in Fig. 42. The accuracy of the velocity field determination for PIV is limited by the ability of the scattering particles to follow the instantaneous motion of the continuous phase. A compromise between reducing the particle size to improve flow tracking and increasing the particle size to improve light scattering is, therefore, necessary. Uniform seeding size is also desirable in order to 


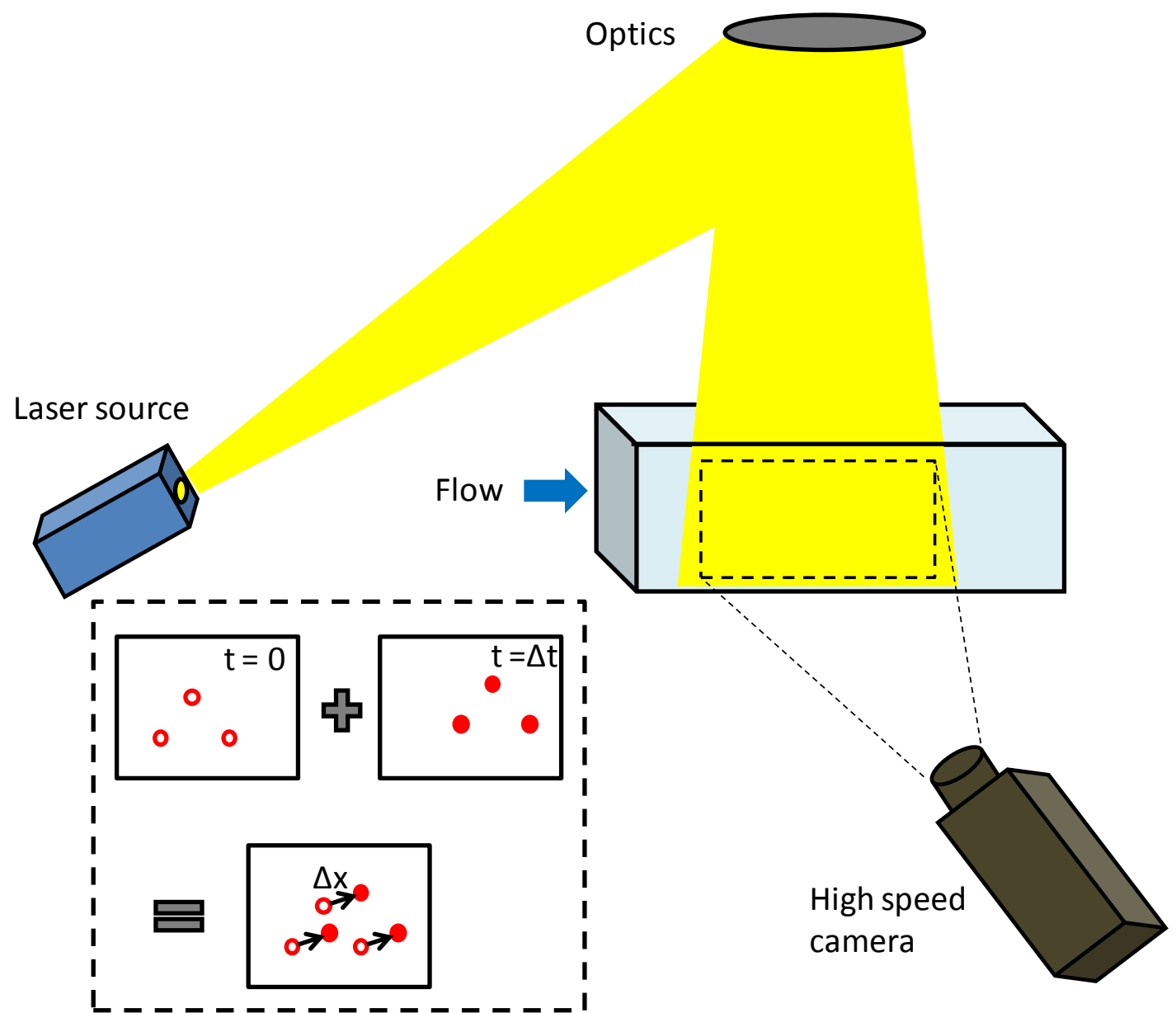

Fig. 42 Schematic of experimental setup for particle image velocimetry. Inset shows a schematic of algorithm used in PIV.

avoid excessive intensity from larger particles and background noise, decreasing the accuracy from small particles. The choice of optimal diameter for seeding particles is a compromise between an adequate tracer response of the particles in the fluid, requiring small diameters, and a high signal-to-noise ratio (SNR) of the scattered light signal, necessitating large diameters [45]. It is also necessary that the densities of the fluid and the tracer particle match, so that gravitational forces on the tracer particles do not alter 
the flow field [44]. Typical tracer particles used for liquid flows are fluorescently labeled polystyrene latex (PSL) [46], aluminum flakes, hollow glass spheres, oils and oxygen bubbles [44].

\section{EXPERIMENTAL SETUP FOR PIV}

Our imaging system consists of a nine nanosecond pulsed laser from Quanta-Ray (Spectra-physics), coupled with two high-speed CMOS cameras system capable of frame rates up to $4.8 \mathrm{kHz}$ at full $800 \times 600$ resolution (and up to over $100 \mathrm{kHz}$ at reduced resolution) with up to 14 bit sensitivity and adjustable shutter speeds as fast as $2 \mu \mathrm{s}$ (Phantom v7.1 and v7.3; Vision Research, and APX LM; Photron). Ultra-zoom lenses (Moritex) were attached to the cameras to obtain high spatial resolution images of the flow field. A laser light sheet was formed and directed to illuminate the experimental test section utilizing high-energy mirrors and beam shaping optics. With this capability it was possible to acquire thousands of image pairs within milli- to micro-second time intervals, thereby enabling the use of advanced ensemble correlations to provide time resolved reconstruction of the full velocity field down to the sub-pixel level. The 2D velocity field was then reconstructed from the camera images. The setup also consisted of the aluminum heater used in the PCR experiments. The test section used in our PIV experiments is shown in Fig. 43. The initial device used for the PCR chamber for the purpose of performing PIV was a plexiglas chamber with holes drilled on to the edges of the rectangular block. 
(a)

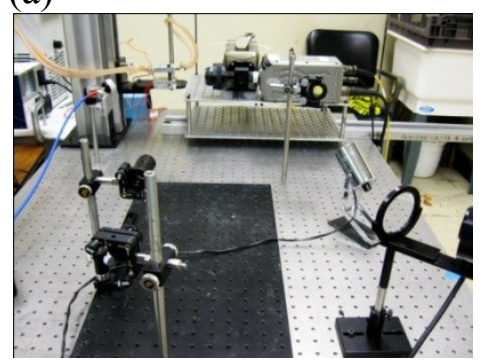

(d)

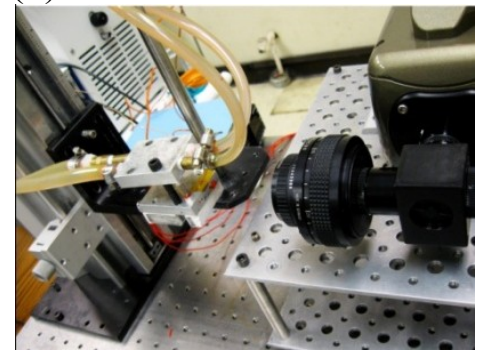

(b)

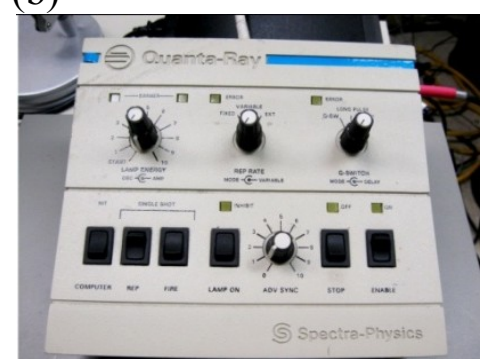

(e)

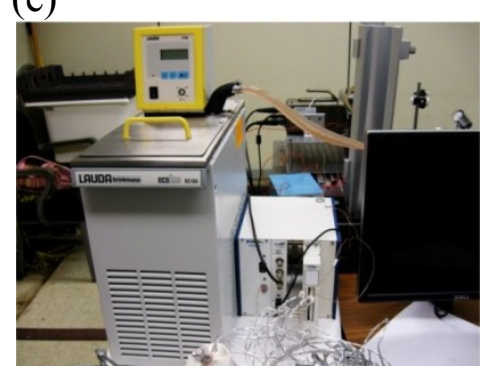

(c)

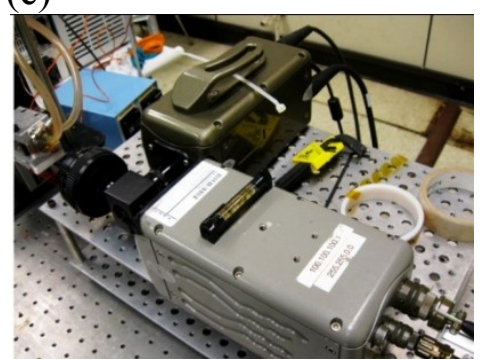

(f)

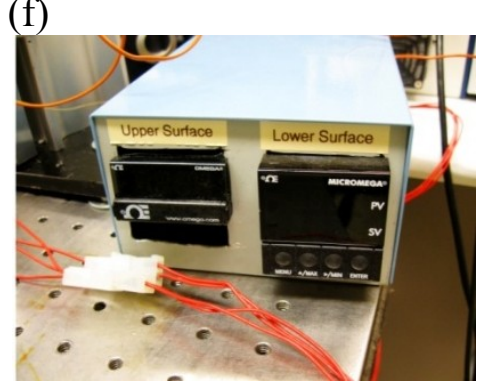

Fig. 43(a) Overview of experimental setup used for PIV. Fig. 43(b) and (c) Quanta-Ray laser controller and cameras used for PIV. Fig. 43(d) Close-up of test section used for the experiment. Fig. 43(e) and (f). Heaters used for the bottom and top surfaces of the test section.

It was observed that this was not very promising since the optical quality of the plexiglas cylinder was not suitable for flow visualization using PIV. Another possibility was to use cylindrical blocks, created from plexiglas, with a thin cylinder drilled at the center, as shown in Fig. 44(a). The thin walls of the cylinder aid in the visualization process. Though we were able to see the particles moving inside the cylinder, we found that the surface finish of the inner cylinder made it difficult to spot a particle hidden behind the rough surface of the cylinder. This is shown in Fig. 44(b).

A new idea was envisioned where the idea was to first create a rectangular mold with the dimensions matching those of the aluminum heater, followed by creating the rectangular prototype with the cylindrical holes using polydimethylsiloxane (PDMS), as 
(a)

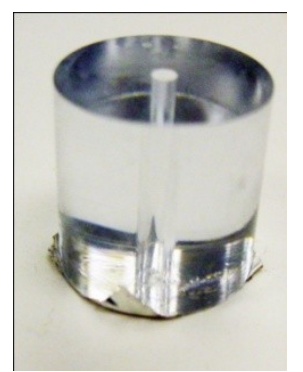

(b)

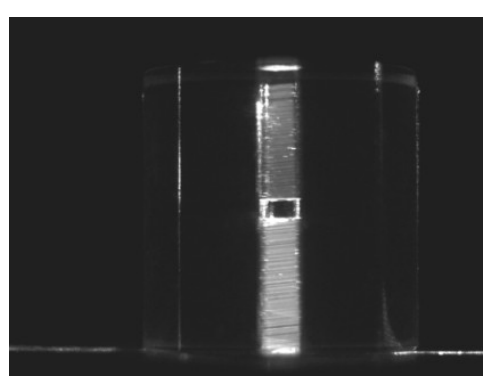

Fig. 44(a) Cylindrical plexiglas block with an inner cylinder drilled at its center. Fig. 44(b) Image obtained from PIV setup, showing the insufficient machine finishing for the inner cylinder. One can also notice the presence of an air bubble in this picture.

shown in Fig. 45.

A brief description of this process is given here. A mixture of Sylgard ${ }^{\circledR} 184$ Silicone elastomer base and curing agent was used in the ratio of 1:10 (by mass). This mixture was vigorously stirred with a metal spoon for $5 \mathrm{~min}$. The next step is to remove the air bubbles, which was done by subjecting the mixture to vacuum conditions and normal atmospheric conditions repeatedly, until all the air bubbles were removed. The plexiglas PCR cartridge (used as the mold) was placed in a glass bowl and the PDMS mixture was poured around it. It was then placed on a hotplate and set to cure for 2 hours at $80{ }^{\circ} \mathrm{C}$. After the mold was formed, it was allowed to cool and the plexiglas cartridge was then removed. The next stage is to create the PDMS cartridge with cylindrical holes around the edges, using the newly created mold. For this purpose, holes were drilled into a plastic block, and dowel pins were inserted so that they could serve as the molds for the cylindrical holes. This arrangement is shown in Fig. 45(b). A new mixture of the PDMS elastomer base and curing agent was then poured into the new setup, using the 
(a)

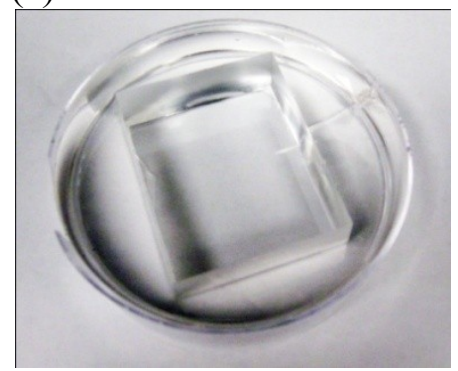

(c)

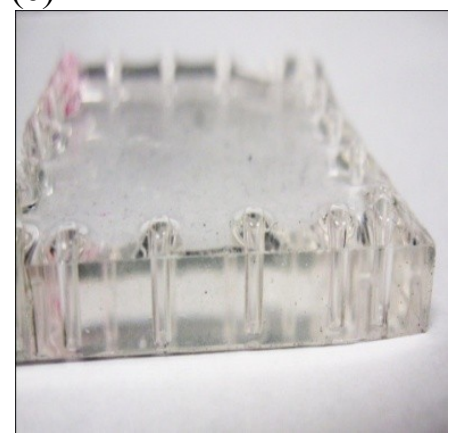

(b)

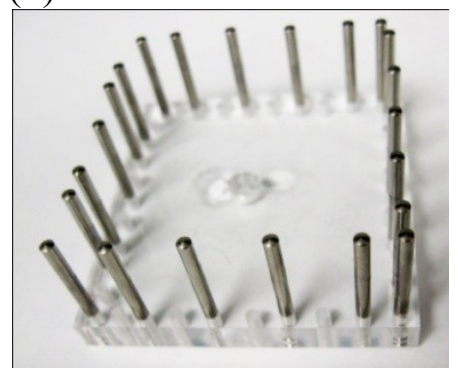

(d)

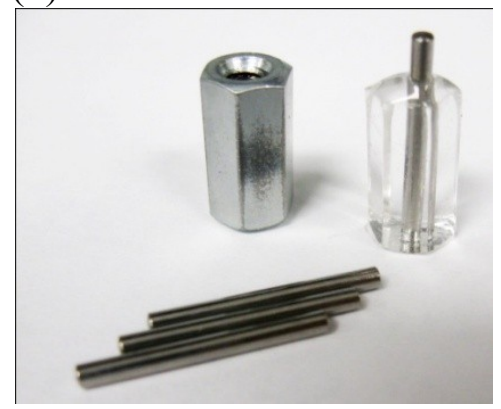

Fig. 45(a) A rectangular mold made up of PDMS, made from a plexiglas rectangular mold, shown in Fig. 45(b). Fig. 45(c) Final product made up of PDMS with holes formed using the dowel pins in the rectangular mold, shown in Fig. 45(b). Fig. 45(d) Hexagonal PDMS chambers created using a similar process.

already created PDMS mold and the plastic block with dowel pins. The setup was placed on a hot plate as before, and the final product was obtained, as shown in Fig. 45(c). This process can be used to create PDMS chambers for any geometry, for example, we also created hexagonal chambers with a cylindrical hole at its center. The reason for using the hexagonal geometry was that it would be possible to align the cameras normal to two adjacent planes of the hexagonal chamber, which would help to reconstruct the three dimensional velocity and temperature fields. This schematic is shown in Fig. 45(d).

The advantage with using PDMS is that the inner and outer surfaces of the cylindrical chamber are optically clear to perform PIV/LIF measurements, as opposed to 
creating cylindrical holes using a drill-bit, which makes the inner surface of the cylinder rough, and optically opaque. PDMS is also inert, non-toxic and non-flammable. Yet another advantage with using PDMS is that it can withstand temperatures under the current heating conditions. However, a major problem that was encountered with the hexagonal geometry was the buckling of the chamber when it was sandwiched between the two aluminum heaters. It was also observed that it was difficult to seal the fluid in the PDMS chambers. Therefore, the idea of using cylinders created using PDMS was discarded, and it was found that glass capillary tubes proved to be useful due to their high optical quality and mechanical strength, which prevents buckling between the aluminum heaters. However, it must be expected that the sidewalls will no longer be insulated, but they can affect the flow due to the higher thermal conductivity of glass compared to plastic.

The solution used for PIV experiments contained Rhodamine particles in DI water, so that one can easily visualize the flow field from the motion of the particles. We found that the laser power source was unsteady, with the values from the spectrometer fluctuating from the average value in a Gaussian distribution. A plot of the laser power distribution is shown in Fig. 46. The magnitude of the laser power must be such that it is high enough for the camera sensors to be able to take good images of the flow field, but it must not be too high that it heats the Rhodamine particles and changes their properties. It was found that laser power values with an average of $300-500 \mu \mathrm{J}$ was sufficient to obtain accurate images from the camera. To perform the experiment, first, the aluminum heaters were preheated to the required temperatures. The laser and the associated cooling 


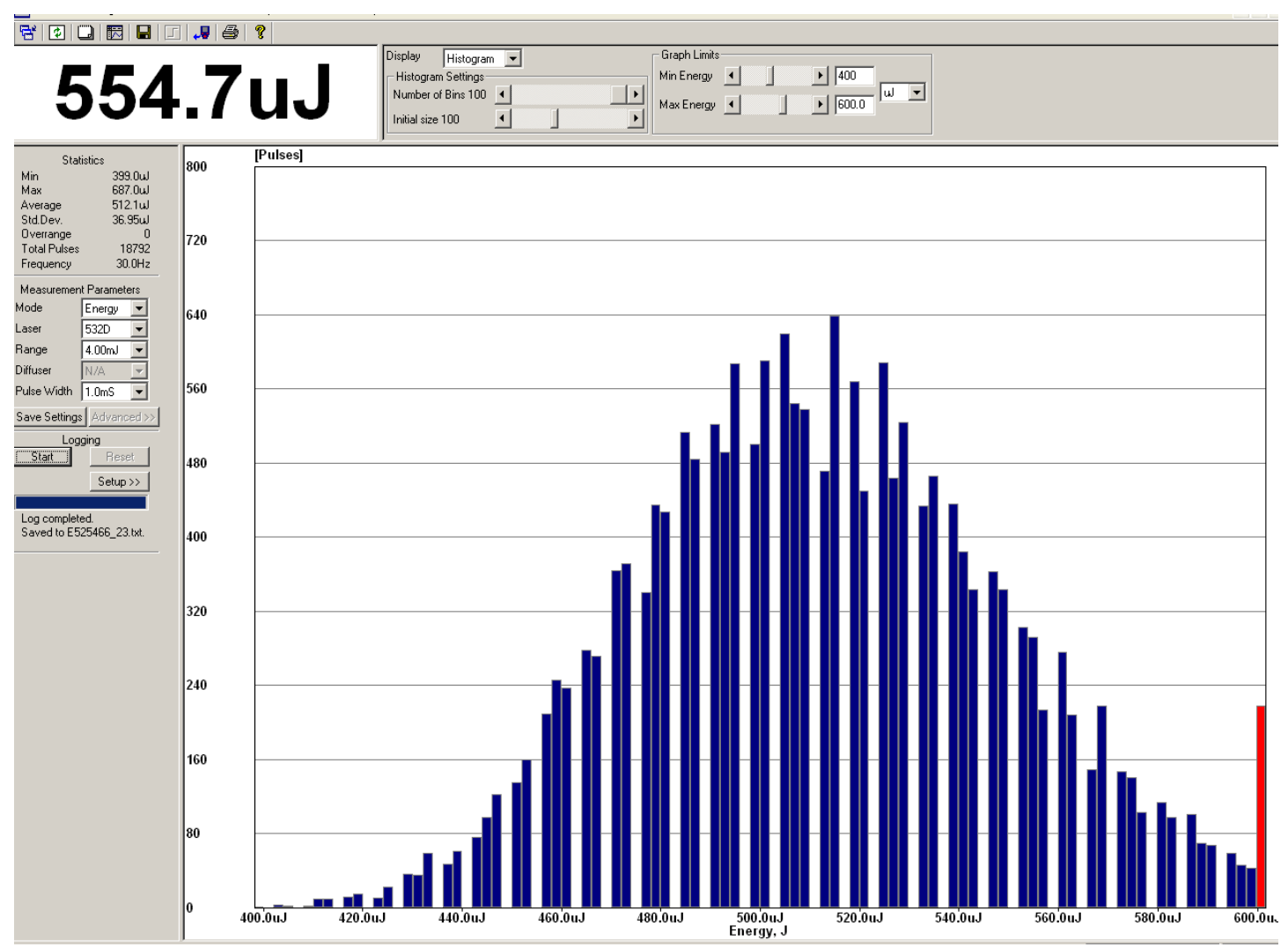

Fig. 46 Distribution of laser power over time, showing a Gaussian distribution of the laser fluctuations.

instrumentation were readied and the glass capillary tube was sealed at the bottom with the Teflon tape, the solution was loaded and the tube was sealed at the top. The test section was sandwiched between the heaters and 3000 images were obtained at a frequency of $30 \mathrm{~Hz}$. Since we lacked a second camera to obtain velocity in the three dimensional plane, we performed 2D PIV for the test section. The experiment was performed for a cylinder of aspect ratio $3.34(\mathrm{~h}=4.63 \mathrm{~mm}, \mathrm{ID}=1.52 \mathrm{~mm}, \mathrm{OD}=7.5 \mathrm{~mm})$. The average value of the laser power for the experiment with aspect ratio 3.34 was $0.4 \mathrm{~mJ}$. 


\section{RESULTS OF PIV EXPERIMENT}

In order to analyze the PIV results, the in-lab program PTV-TAMU was used. The first step in analyzing the images is to create a candidate window of appropriate size, centered at the particle, which defines how many pixels a particle moves - a very small size window is needed if the particles barely move and a large size window is needed if the particles have high velocity. Another window is also defined called the correlation window which then allows one to perform cross-correlation between successive images to find the new location of the particle, and eliminate erroneous predictions of particle movement (that is, cross correlation is performed between different sets of particles, and the pair of particles with the highest value is chosen as the correct pair). The range of values of the cross correlation coefficient is between -1 to +1 , with +1 being the highest value possible. A correlation threshold also helps so that any particle combination that results in a value less than the correlation threshold can be immediately discarded, without needing further investigation. If the value of the correlation threshold is too low, then it is possible to obtain more vectors, but the accuracy will be compromised. Thus, it is important to maintain a high value for the correlation threshold. The next stage is to create images with ideal shapes of particles, so that particle tracking is performed using the centroids of the particles. Here, a particle radius is chosen, along with the size of the window containing the particle. Cross correlation is now performed to find locations of particles. A grey scale threshold value is also chosen to remove background noise. Finally, one can choose to skip between successive images, in order to obtain reasonable velocity vectors. This process is 
automated in the in-house PTV software which was used to obtain the velocity vectors from the PIV experiment. One can also obtain various statistics like average of instantaneous velocity, vorticity, etc. using the software.

A typical PIV image is shown in Fig. 47(a). The bottom heater was maintained at a temperature of $60{ }^{\circ} \mathrm{C}$ and the top heater was maintained at $17{ }^{\circ} \mathrm{C}$. Fig.47(b) and (c) show the contour plot and vector plot of velocity long with the streamtrace, taken at the plane illuminated by the laser. One can observe that the flow seems to be organized and periodic, not chaotic. The flow velocity is also very small in magnitude (order of $10^{-5}$ ). This can be attributed to the dimensions of the cylinder and the boundary conditions, which places this test section close to the regime of the critical Rayleigh number for onset of convection. The flow seems to be more localized at the bottom half of the cylinder.

\section{COMPARISON OF NUMERICAL RESULTS WITH PIV EXPERIMENT}

In order to compare the results of the PIV experiment with numerical simulations, a conjugate model was created in GAMBIT, where the fluid and solid portions of the cylinder were created $(\mathrm{h}=4.63 \mathrm{~mm}, \mathrm{OD}=7.5 \mathrm{~mm}, \mathrm{ID}=1.52 \mathrm{~mm})$. Glass was modeled with a density of $2600 \mathrm{~kg} / \mathrm{m}^{3}$, specific heat of $840 \mathrm{~J} / \mathrm{kgK}$ and a thermal conductivity of $1.05 \mathrm{~W} / \mathrm{mK}$. The top and bottom surfaces of the cylinder were heated to 17 and $60{ }^{\circ} \mathrm{C}$, and the sidewalls were modeled as being exposed to air at $20^{\circ} \mathrm{C}$, with a heat transfer coefficient of $10 \mathrm{~W} / \mathrm{m}^{2} \mathrm{~K}$. We found that we were not able to obtain a 
(a)

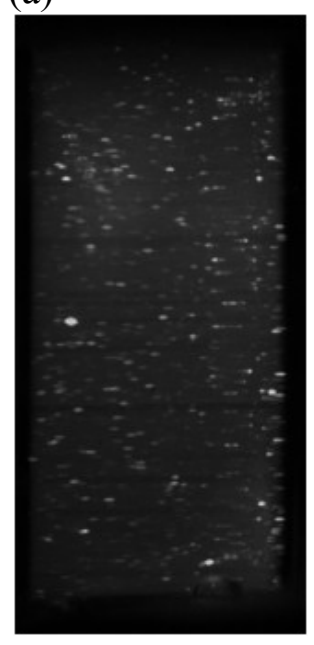

(b)

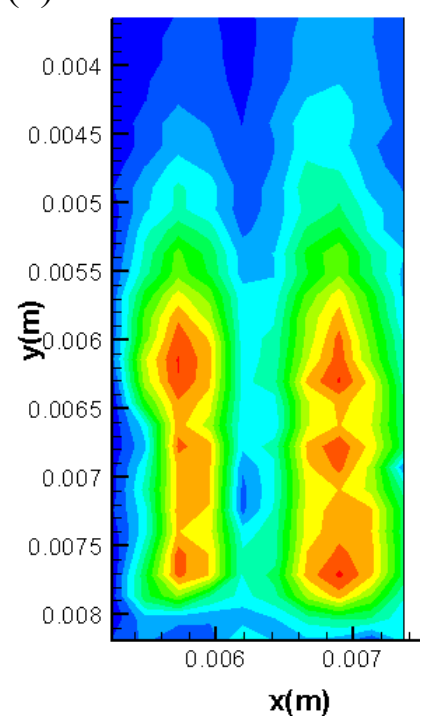

(c)

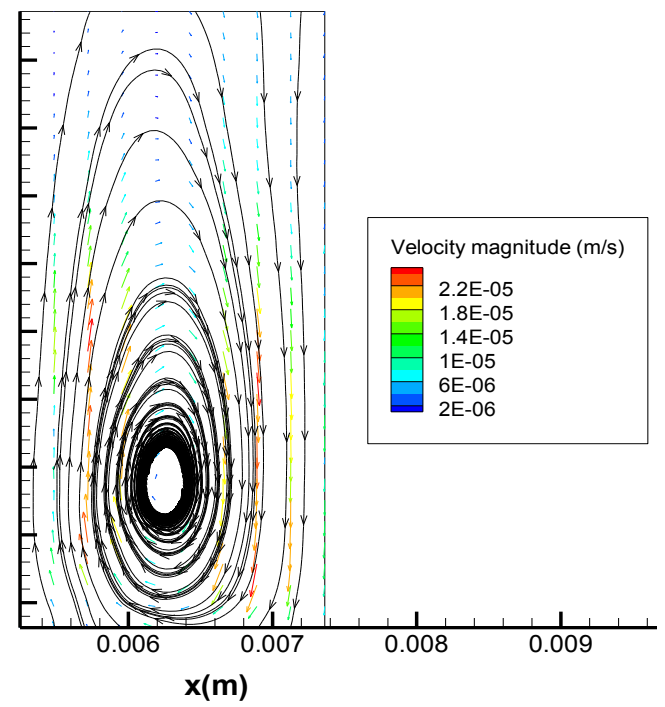

Fig. 47 (a) Typical image obtained from the PIV experiment. Fig. 47(b) Contour plot of velocity for the fluid in the experiment. Fig. 47(c) Plot of velocity vectors and streamtrace for the fluid in the experiment.

converged solution with this model. We then tried a different approach where we modeled the fluidic volume in GAMBIT $(\mathrm{h}=4.63 \mathrm{~mm}, \mathrm{~d}=1.52 \mathrm{~mm})$ and we modeled glass as an external volume created in FLUENT through the use of the wall thickness function. The boundary conditions were modeled as before. We found that using an unsteady solver helped to model the formation of flow from a stationary stage. We found that using this method, we obtained a solution that agreed in part with our experimental results. Our solution obtained at time $t=40$ seconds matched with the experimental results obtained from PIV (as shown in Fig. 48). However, this result is one that is transient, the simulations show that a fully developed flow is obtained at time $\mathrm{t}=56$ seconds. Therefore, though our simulation results match with those obtained from PIV, these results are at best, preliminary results, since it was possible that the flow was not 
fully developed during the experiment.

(a)

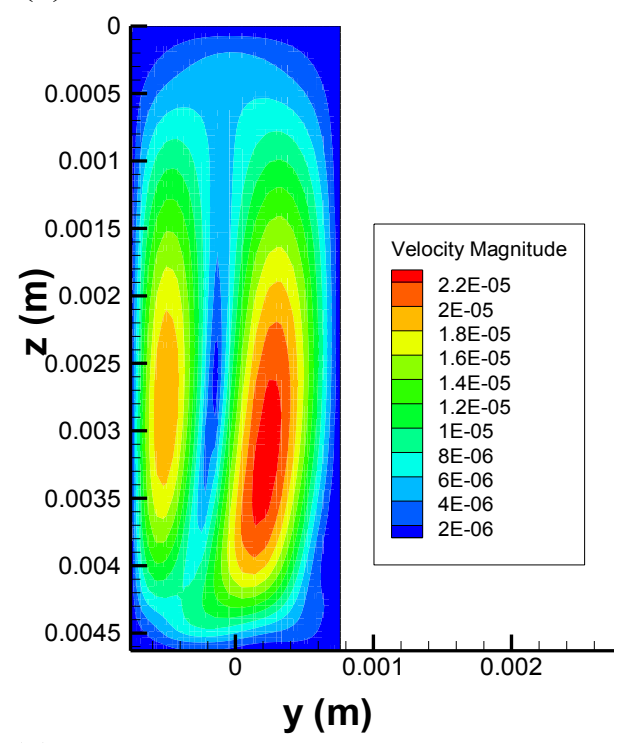

(c)

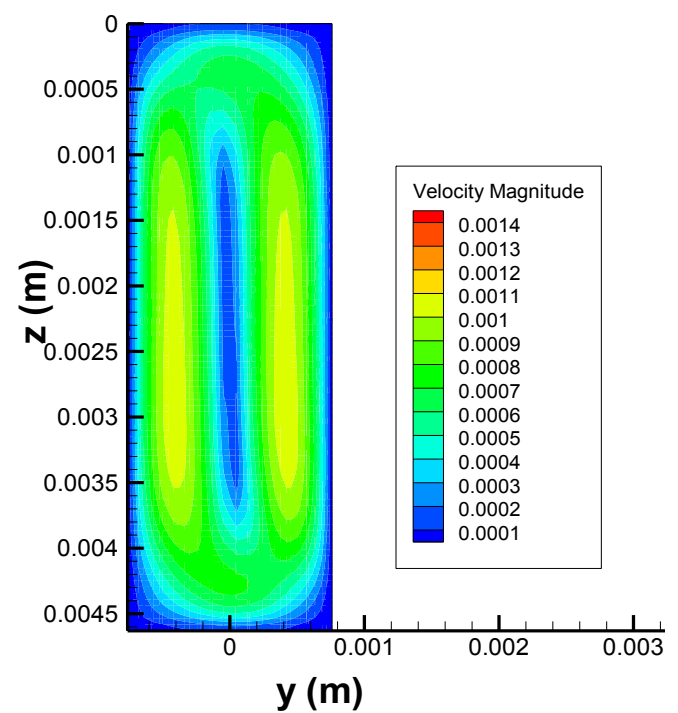

(b)

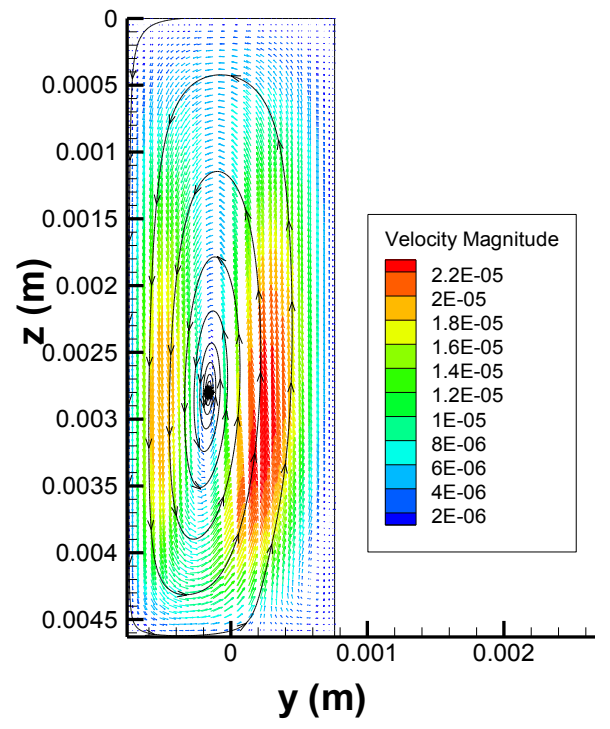

(d)

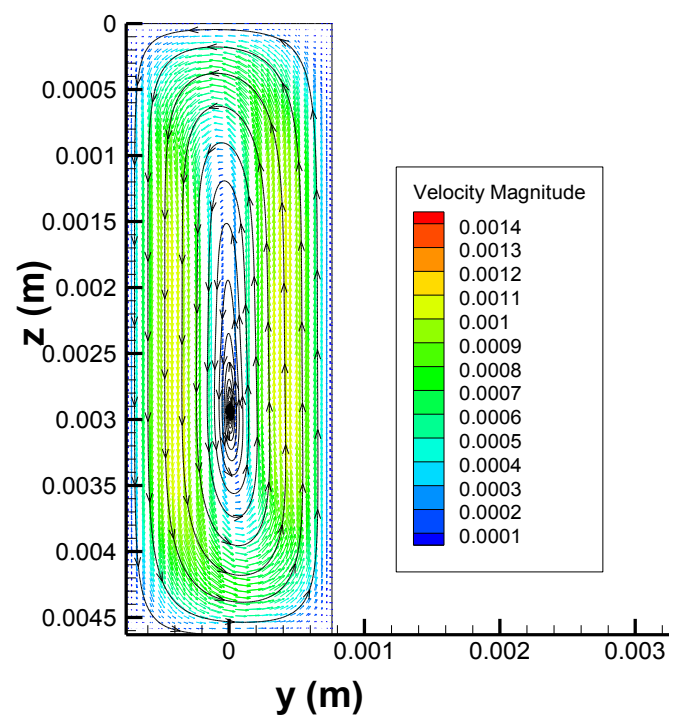

Fig. 48(a) and (b) Contour and vector plots of velocity for the numerical simulation taken at time $\mathrm{t}=40$ seconds. Fig. 48 (c) and (d) Contour and vector plots of velocity for the numerical simulation taken at time $\mathrm{t}=56$ seconds. 


\section{INTRODUCTION TO LASER INDUCED FLOURESCENCE}

Laser-induced Fluorescence (LIF) is an optical, non-intrusive technique used for computing the temperature distribution in a flow field. The fluid is seeded with a fluorescent dye and the temperature of the flow field is obtained when the dye is excited by a laser light tuned into the excitation wavelength of the dye molecules. [43]. A typical LIF setup is similar to that used for PIV - it essentially consists of a source of light (usually a laser), an arrangement of lenses to form a laser sheet, fluorescent medium and

a detector. Typically, the lasers used as light sources have short pulse time for good temporal resolution. Timing electronics are also used to synchronize the laser with the cameras. For this reason, it is possible to perform PIV and LIF experiments with the same experimental apparatus, making minor modifications for each experiment.

In our experiments, non-invasive temperature measurements also needed to be performed in order to map out the temperature profile of the flow field at a wide range of heating conditions. This is possible by imaging seed particles that exhibit temperatureinduced changes in color or fluorescence using LIF. Thermochromic liquid crystals (TLCs) have been widely used to map temperature distributions in a variety of flows, but the temperature range of sensitivity is usually only $\sim 10{ }^{\circ} \mathrm{C}$ [47]. Fluorescent dyes such as Rhodamine $\mathrm{B}(\mathrm{RhB})$ have also been used to perform in-situ temperature measurements in flow fields [48]. Rhodamine $\mathrm{B}$ is particularly attractive because its spectral characteristics are such that it absorbs light in the green wavelength range, which is an ideal match to the Nd:YAG lasers employed in our PIV system. It belongs to the red emitter class, and its fluorescent intensity exhibits a measurable response over the entire 
temperature range relevant for PCR, which is suitable for also performing LIF experiments.

\section{EXPERIMENTAL SETUP FOR LASER INDUCED FLOURESCENCE}

In order to perform the LIF experiments, the solution that was used for the experiment contained $1.6 \mathrm{mg} / \mathrm{L}$ of Fluorescein and $0.8 \mathrm{mg} / \mathrm{L}$ of Rhodamine. The reason for using two different chemicals in the solution is that as the temperature of the solution increases, the fluorescence of Fluorescein increases, while that of Rhodamine decreases. Thus it is possible to take images of the solution with two cameras, one having the Rhodamine filter and the other with the Fluorescein filter. The images can then be processed together to obtain the temperature distribution of the flow field - this procedure ensures that the final result is independent of the intensity of the laser light.

Before performing the experiment, it was necessary to evaluate the effect of the laser on the solution, i.e. it is possible that the laser power is too strong for the solution of given concentration and volume, so it is possible that the laser can photobleach the solution. For this purpose, analysis was performed using a spectrometer - the test section was assembled, and the heaters were not used, i.e. the solution was at room temperature. The laser was switched on and data was obtained every five min - the plotted data with the intensity of the fluorescence on the y-axis and the wavelength on the $\mathrm{x}$-axis is shown in Fig. 49. The excitation wavelength for Rhodamine is $580 \mathrm{~nm}$, for Fluorescein is $528 \mathrm{~nm}$, and for the laser power is $532 \mathrm{~nm}$. These are observed as three different peaks in Fig. 49. It can be seen that the difference in laser intensity is more prominent for 


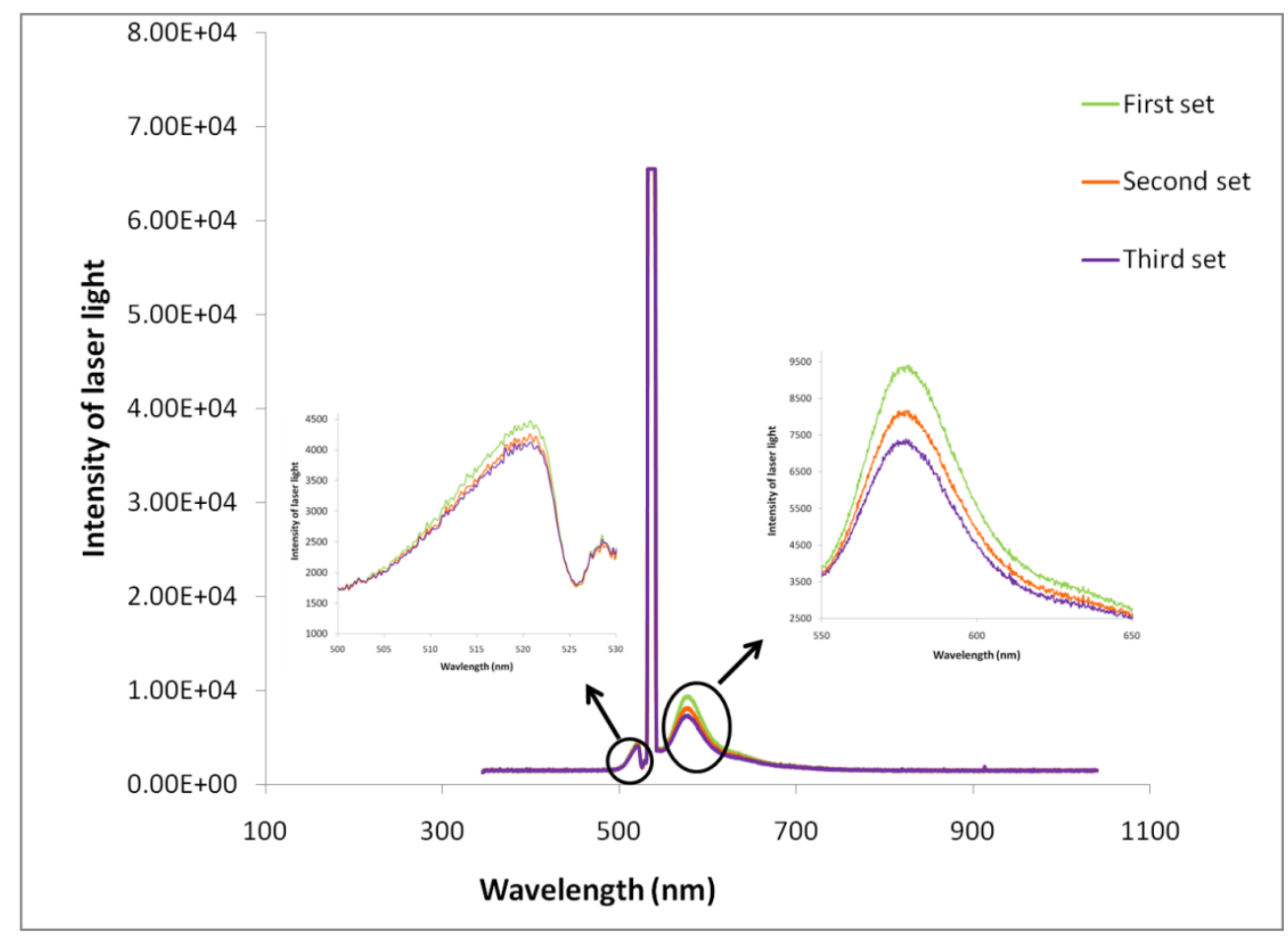

Fig. 49 Intensity of the light recorded by the spectrophotometer with concentration of Fluorescein $1.6 \mathrm{mg} / \mathrm{L}$, and for Rhodamine $0.8 \mathrm{mg} / \mathrm{L}$. The insets shows the magnification of the individual peaks corresponding to Fluorescein and Rhodamine.

Rhodamine than Fluorescein - the difference between the heights of the first and last peak for Rhodamine is about $21 \%$. From Fig. 49 , it can be observed that the intensity of fluorescence decreased as a function of time, despite the fact that the experimental conditions remained the same. This was due to the fact that laser power was too strong for the fluorescent particles - however, decreasing the power of the laser any further results in under-exposed images. In order to overcome this dilemma, an antiphotobleaching agent was added to the solution - in the current case, we used 2mercaptoethanol to suppress the photobleaching. We found that we had loading issues with this solution - the solution doesn't adhere to the walls of the glass cylinder, thus 
leading to formation of air bubbles. Another idea to overcome the photobleaching issue was to increase the concentration of Fluorescein and Rhodamine at least ten-fold so that any photobleaching will have a negligible impact in the overall fluorescence. We found that increasing the concentration of Rhodamine to $10 \mathrm{mg} / \mathrm{L}$ and Fluorescein to $20 \mathrm{mg} / \mathrm{L}$ reduced the effect of photobleaching considerably - this was due to the fact that the concentration of the fluorescent particles was extremely high, so that even though the laser could photobleach some part of the fluorescent molecules, the overall effect of photobleaching is negligible. This idea was found to work well in our experiments, as shown in Fig. 50. Here, the difference between the peaks for Rhodamine was about 7\%, which is more acceptable than before. This idea is further illustrated in Fig. 51, which shows a series of images taken of the test-section, with the Rhodamine filter, at different periods of time, with the incident laser maintained at a constant value. This example shows that the laser is not able to photobleach the solution significantly, as the intensity of light emitted from the solution is constant.

One problem that was encountered during the experiments was the sudden appearance of air bubbles in the chamber - this is also visible from Fig. 51. This could be because the bond between the Teflon tape and the glass cylinder was not very strong. In order to overcome this issue, an idea was developed where metal caps could be fabricated which could seal the openings on the top and bottom of the cylinder. The metal cap was made of aluminum. In order to ensure that there is no leakage between the metal cap and the glass capillary, glue that was stable at high temperatures was applied on the surface of the metal cap and then it was inserted into the cylindrical hole and left 
to cure for one hour. This was followed by loading the solution into the chamber, and then sealing the top using another metal cap. However, we were surprised to find that the fluorescence solution was getting bleached during the experiment. We assumed that this could be because of the glue, so we tried to seal the metal cap using superglue instead. Once again, we observed that the solution was photobleached. We concluded that perhaps the metal caps were affecting the solution and therefore, this idea was discarded. We continued to use the Teflon tape, and repeated the experiment if an air bubble found its way inside the cylindrical chamber. In order to perform LIF experiments to map out the temperature regimes in the Rayleigh-Bernard system, it is necessary to first obtain

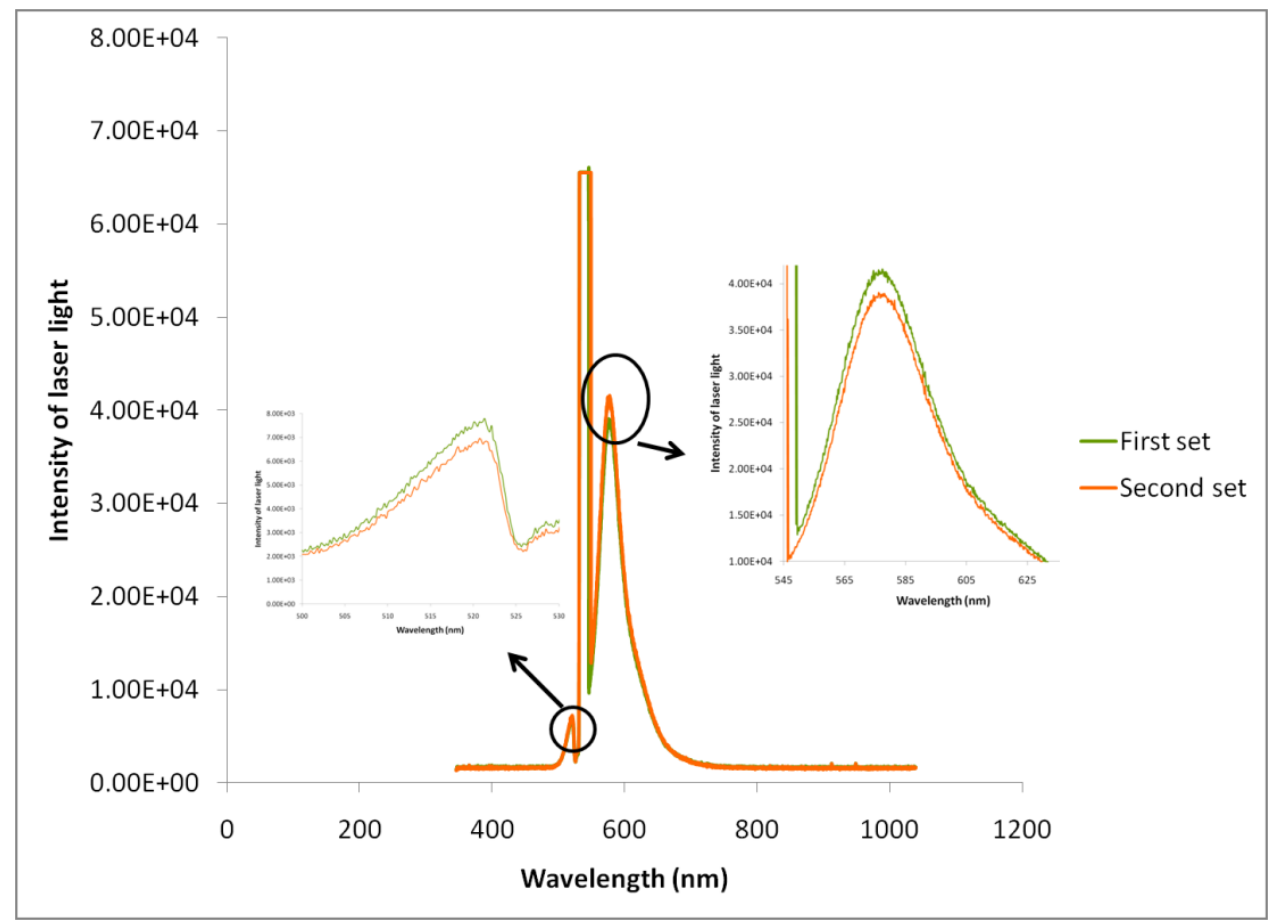

Fig. 50 Intensity of the light recorded by the spectrophotometer, with concentration of Fluorescein $20 \mathrm{mg} / \mathrm{L}$, and for Rhodamine $10 \mathrm{mg} / \mathrm{L}$. The insets shows the magnification of the individual peaks corresponding to Fluorescein and Rhodamine. 

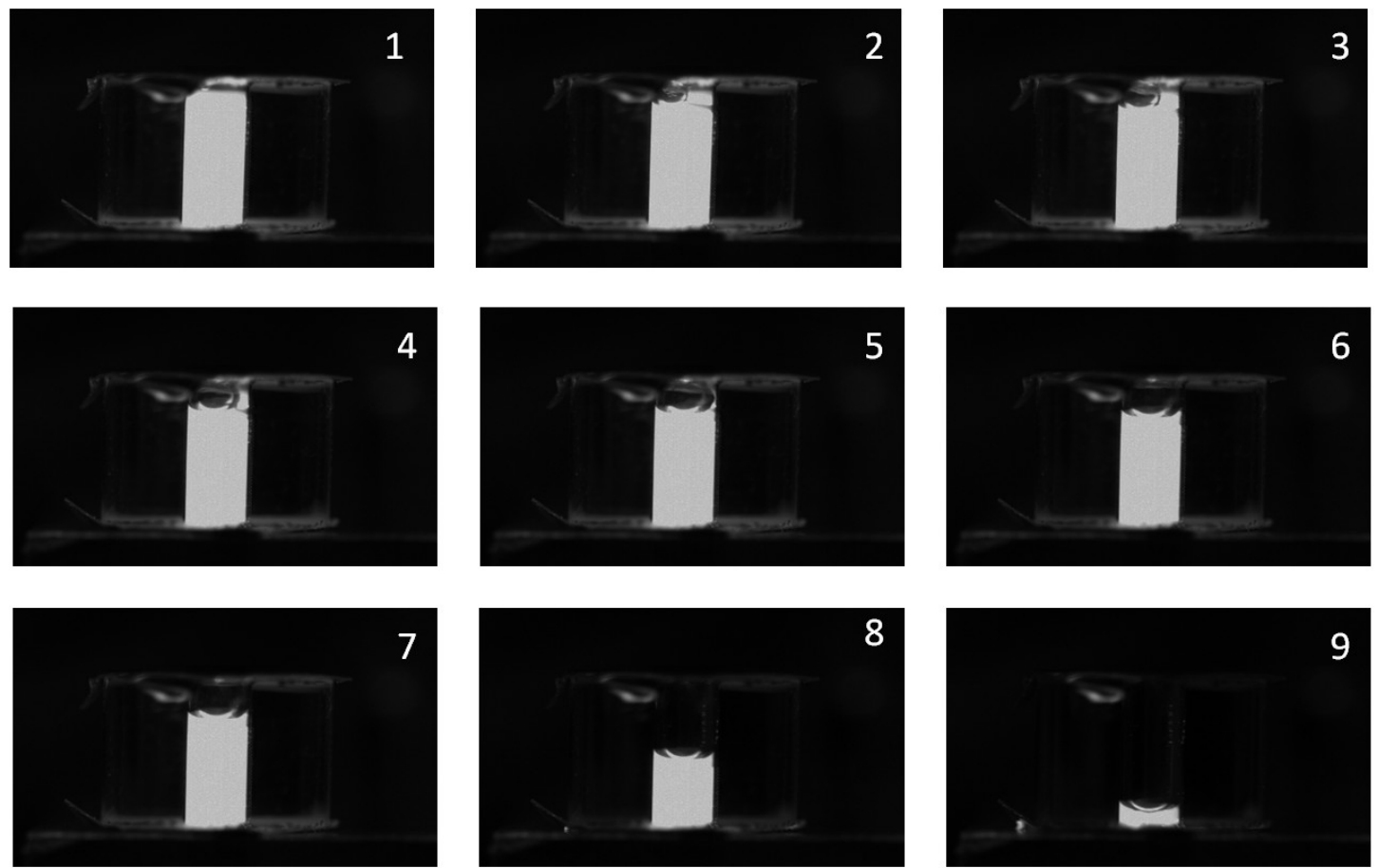

Fig. 51 Images taken from the LIF experiment, illustrating the negligible photobleaching effect of the laser on the solution. It also illustrates the problems that were encountered due to the presence of air bubbles in the solution.

calibration data, where the cylinder with the fluid is maintained at a constant temperature, i.e. the top and bottom heaters are kept at the same temperature and then the fluid is illuminated with the laser and a sequence of images are obtained. This experiment is performed for different sets of temperatures. We obtained data at $24{ }^{\circ} \mathrm{C}$, $30{ }^{\circ} \mathrm{C}, 40{ }^{\circ} \mathrm{C}$ and $50{ }^{\circ} \mathrm{C}$. Then, without disturbing the setup or changing the working fluid, the actual experimental data is obtained by maintaining the top heater at $10{ }^{\circ} \mathrm{C}$ andthe bottom heater at $50{ }^{\circ} \mathrm{C}$. We did not use higher temperatures for the bottom heater as we noticed formation of bubbles in the fluid, which would disturb the fluid motion. We performed these experiments for the cylinder with aspect ratio 3.34. 


\section{RESULTS FROM LIF EXPERIMENT}

In order to analyze the images from the LIF experiment, we used another inhouse software developed for LIF. It is first necessary to obtain an image which contains the average of all the images of that experiment - this is done separately for both the Rhodamine and Fluorescein images. Now a new set of images is obtained which is a ratio obtained by dividing the Rhodamine images with the Fluorescein images, in order to cancel any effects due to laser fluctuations. A meshed region is now defined for calibration for the new images and a calibration curve (which is a polynomial of desired order) is obtained using the experimental data obtained at constant upper and lower surface temperatures. This curve is then used to predict the temperatures of the experimental data which contains a temperature gradient between the lower and upper surfaces.

A set of typical LIF image are shown in Fig. 52 (a) and (b) taken with the Rhodamine and Fluorescein filter, respectively - both the images were obtained with the top and bottom temperatures maintained at $24^{\circ} \mathrm{C}$. The analysis for the LIF results was performed by creating a grid on the image and calibrating the images with an nth order polynomial. Fig. 52 (c) shows the temperatures along the mid plane along the height of the cylinder, with the length along the $\mathrm{x}$ and $\mathrm{y}$-axis divided into 30 and 90 cells respectively, for various orders of polynomials. It can be seen that a polynomial of order 6 is sufficient to predict temperatures for the current LIF images. Using polynomials of order greater than 6 yielded negative temperatures - these polynomials were disqualified immediately. Using a polynomial of order 6 , a grid convergence study was performed 
for different grid sizes $-30 \times 90,40 \times 120$ and 50x150 cells. It can be observed from Fig. 52(d) that a grid size of $50 \times 150$ is sufficient to predict temperatures for the current

(a)

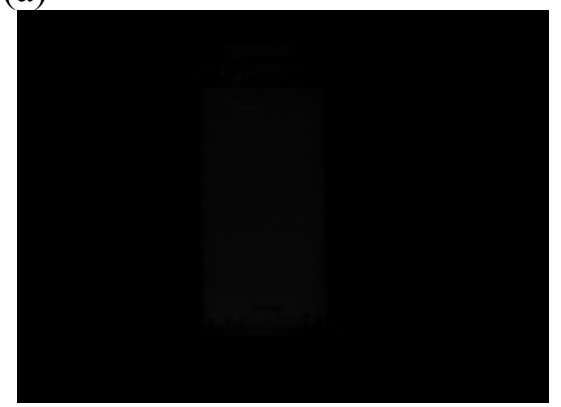

(c)

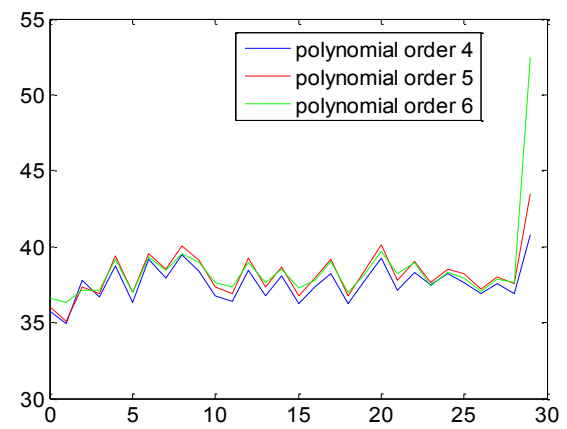

(b)

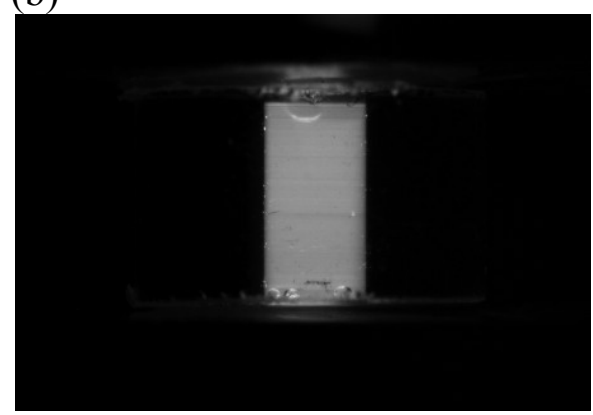

(d)

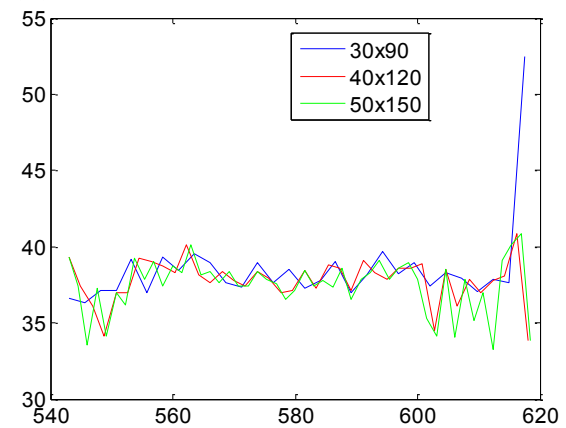

(e)

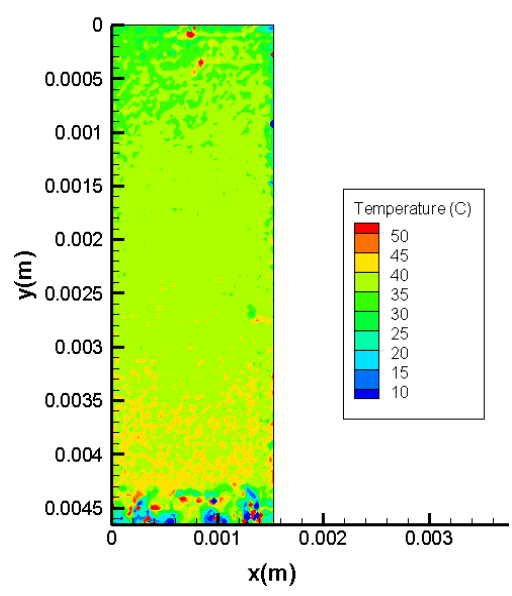

Fig. 52 (a) and (b) Sample images from the LIF experiment, taken with the Fluorescein and Rhodamine filters, respectively. Fig. 52(c) Polynomial convergnce performed for constant gird size. Fig. 52(d) Grid convergence performed for constant polynomial order. Fig. 52(e) LIF image obtained with the top and bottom surfaces maintained at 10 and $50{ }^{\circ} \mathrm{C}$. 
LIF images. Fig. 52(e) shows the LIF image for the cylinder of aspect ratio 3 maintained between 10 and $50^{\circ} \mathrm{C}$.

\section{NUMERICAL SIMULATIONS OF LIF EXPERIMENT}

We performed numerical simulations to obtain temperature contours which could be used to compare with the experimental results obtained from LIF. We modeled the geometry with the top surface maintained at $10{ }^{\circ} \mathrm{C}$ and the bottom surface at $50{ }^{\circ} \mathrm{C}$, with the sidewalls defined with wall thickness and exposed to air at $20^{\circ} \mathrm{C}$, with a heat transfer coefficient of $10 \mathrm{~W} / \mathrm{m}^{2} \mathrm{~K}$. The temperature contours for the simulation results are shown in Fig. 53. The results deviate from the experimental results at the top and the bottom surfaces of the cylinder. This is because the method of analysis does not resolve the thermal boundary layers due to the magnification of the cameras and lenses that were used in the experiment. Furthermore, the interface of solid and liquid surfaces leads to the reflections from the laser. Another problem that we encountered was the presence of
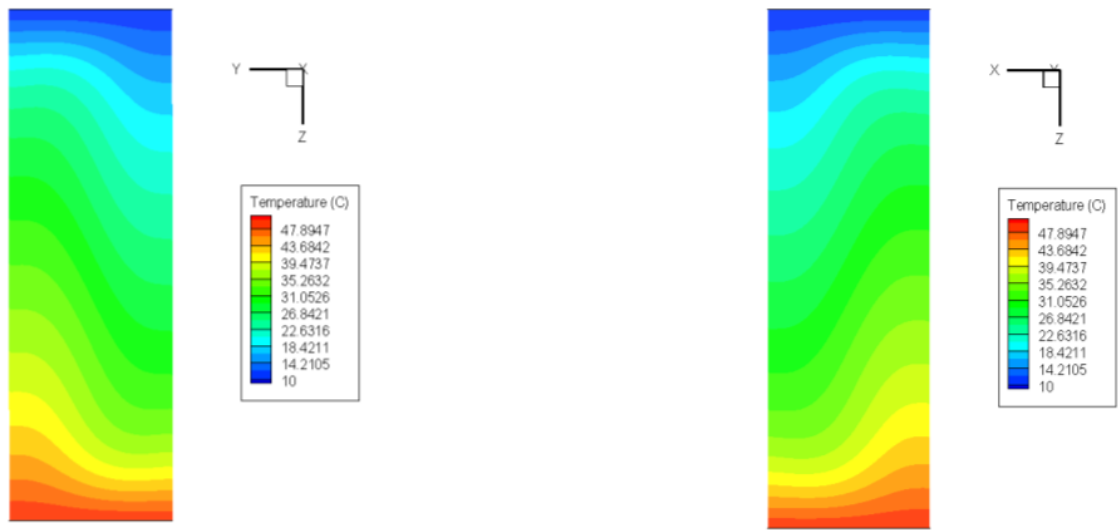

Fig. 53 Temperature contours from the numerical simulations of the LIF experiment in the $\mathrm{YZ}$ and $\mathrm{XZ}$ planes. 
air bubbles at the top and bottom surfaces of the cylinder, as seen in Fig. 53(b). All these factors affect the accuracy of the experimental results. However, we can notice the manifestation of the convective flow inside the cylinder in the experiment, evidenced by the non-linear distribution of the temperature fields, which compares well with the simulation.

\section{VISUALIZATION USING OPTICAL MICROSCOPE}

In order to validate our computational fluid dynamics simulations, it was still necessary to perform flow visualization for a geometry with properties similar to a PCR reaction chamber. We found that using highly transparent acrylic as a rectangular block, with holes drilled in it, was promising because of its highly transparent surfaces. The thickness of the rectangular block is $5.9 \mathrm{~mm}$ and the diameter of the holes drilled is 1.95 mm. We used Fluoresbrite plain YG $10 \mu$ microspheres (2.5\% solids-latex) from Polysciences, Inc. as fluorescent particles. The excitation and emission wavelengths of these particles is 441 and $486 \mathrm{~nm}$ respectively. We found that using a high concentration of particles makes it difficult to visualize the flow, so a low concentration of particles is preferred for this experiment. However, a question arises - do the particles influence the flow field? This question can be answered by computing the Stokes number of the particle, which is defined as [49]:

$$
S t=\frac{\rho d_{p}^{2} U}{18 \mu D_{P}}
$$


It has been established in literature that [49] if $S t \ll 1$, then the particle can be assumed to be following the flow faithfully. However, if $S t » 1$, then the particle influences the flow and this particle cannot be used for flow visualization purposes. In the current study, for a $10 \mu$ particle, with flow velocity being on the order of $1 \mathrm{~mm} / \mathrm{s}$, and the characteristic dimension being on the order of $10 \mu$, the Stokes number is of the order of $10^{-3}$, thus we can be confident of the ability of the particle to trace the fluid motion accurately.

The setup consisted of an optical microscope (Olympus, SZX12), with an Olympus light source, a set of amplifiers and gain modulators and a monitor. Thus, it is possible to view the real time motion of particles using this methodology. In order to perform the experiment, holes were drilled into the block and the bottom surface of the block was sealed with aluminum tape. The fluorescent solution was loaded and the top surface was sealed using Teflon tape. The bottom surface was heated to $60{ }^{\circ} \mathrm{C}$ and the top surface was cooled to $20^{\circ} \mathrm{C}$. A GFP filter was used to illuminate the particle. A video was obtained of the motion of the particles inside the cylindrical chamber. It is not possible to analyze this video using the in-house PTV software because the concentration of particles is too low and in this experiment, the total volume of the fluid is being illuminated while in the case of PIV, the laser sheet illuminates the central plane of the cylinder. This makes it very difficult to obtain an accurate velocity field. In order to analyze the video, an arbitrary particle was chosen and its motion was recorded every second using a digital ruler, as shown in Fig. 54(a). The length of the cylinder corresponded to $9 \mathrm{~cm}$ on the digital ruler. This method was repeated for a few particles. 
The velocity of the particle was calculated based on its displacement in a span of 1 second. A few representative particle motion profiles are shown in Fig. 54(b)-(e).

(a)

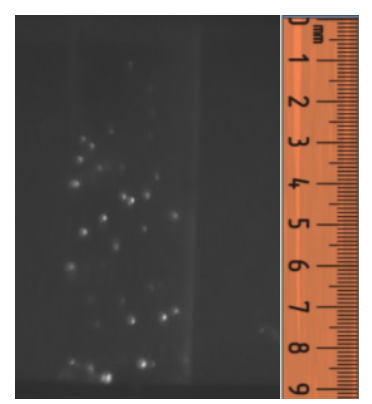

(b)

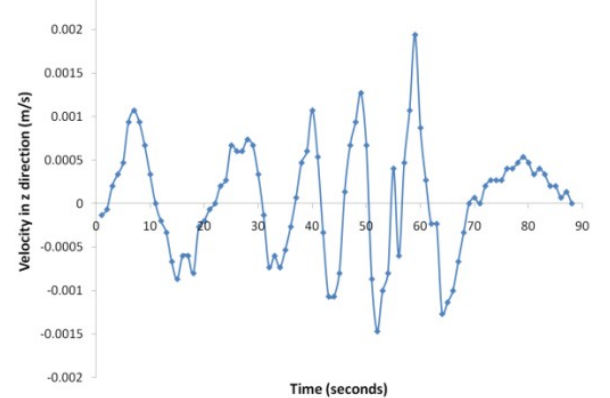

(d)

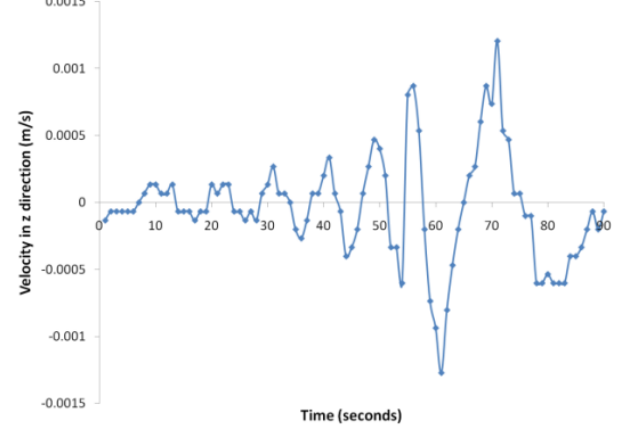

(c)

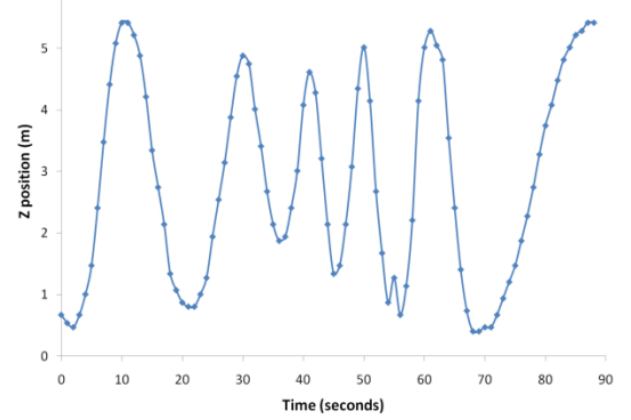

(e)

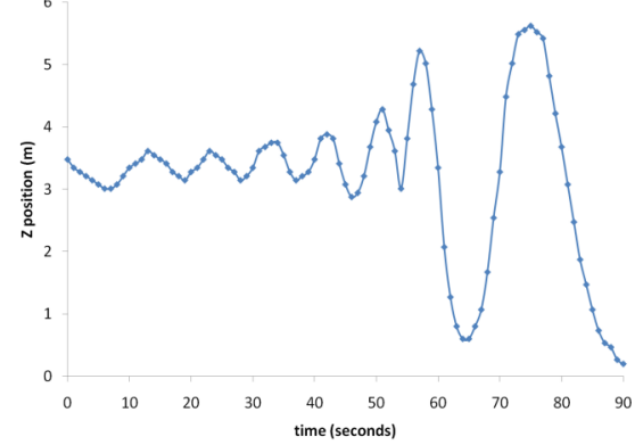

Fig. 54(a) Screenshot of movie with the digital ruler in the background. Fig. 54(b) and (d) Velocity in the $\mathrm{z}$ direction for two arbitrarily chosen particles from the visualization experiment using the optical microscope. Fig. 54(c) and (e) Position along the $z$ direction for two arbitrarily chosen particles from the visualization experiment using the optical microscope. 
The geometry used for the purpose of visualization using an optical microscope was also simulated using GAMBIT and FLUENT. A few typical streamtraces are shown in Fig. 55, with their velocity profiles plotted against time. It can be seen that the velocities are of the same order as that obtained from the experiment. One can also notice similarities between the trajectory of a particle in the experiment and the simulation. This validates our computational fluid dynamics simulations of the PCR geometries.

(a)

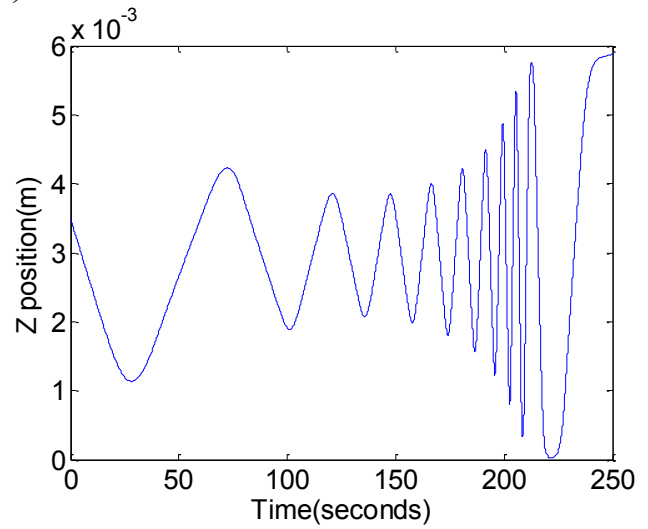

(c)

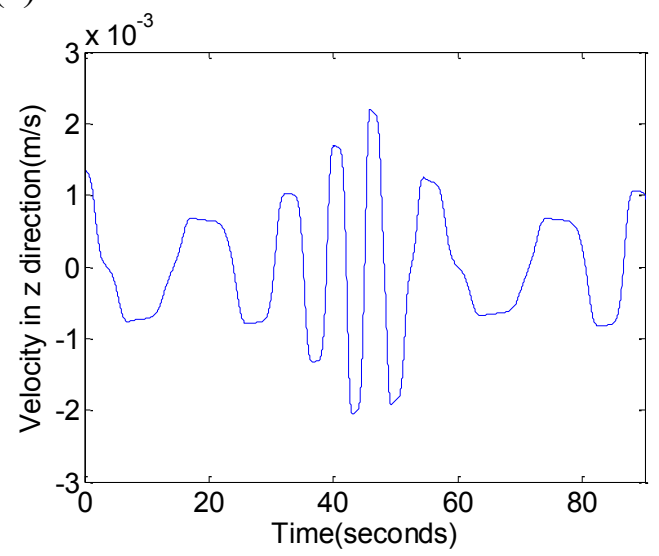

(b)

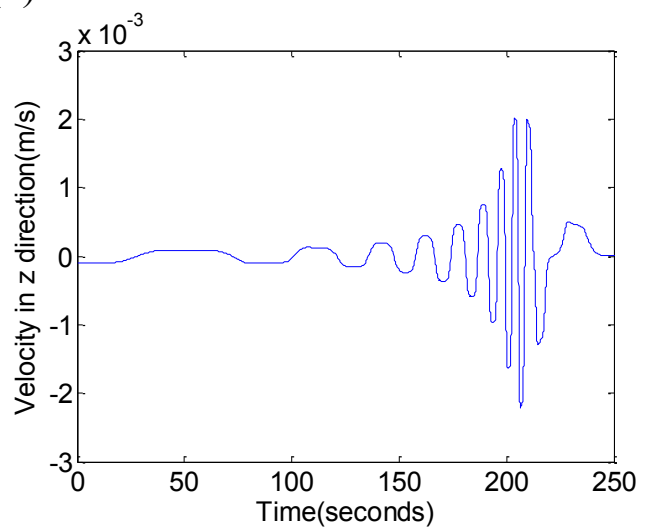

(d)

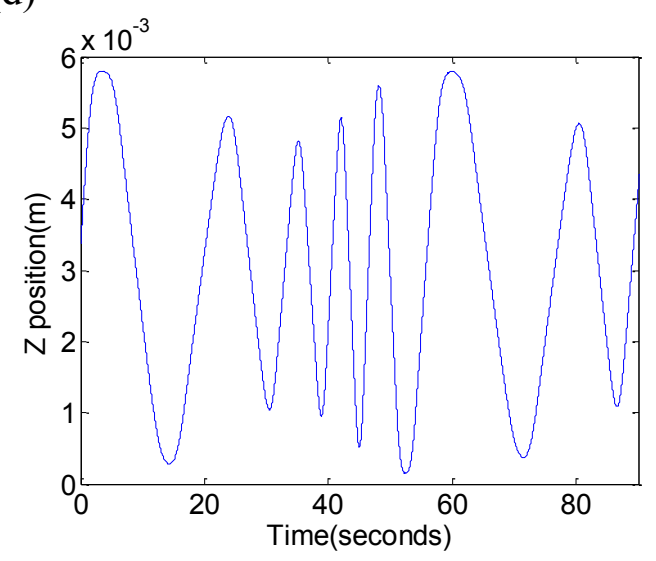

Fig. 55(a) and (c) Velocity in the $\mathrm{z}$ direction for two arbitrarily chosen particles from the numerical simulation of visualization experiment using the optical microscope. Fig. 52(b) and (d) Position along the $\mathrm{z}$ direction for two arbitrarily chosen particles from the numerical simulation of visualization experiment using the optical microscope. 


\section{CHAPTER VI}

\section{CONCLUSIONS}

Numerical simulations were performed for the Rayleigh-Bénard system, with particular applications to polymerase chain reactions. Using CFD, different types of flows were identified and their influence on the rate of the chemical reaction was predicted based on the nature of the flow field. In particular, it was found that using a cylinder with aspect ratio 3 seemed more disordered than the cylinder with aspect ratio 9, which was used in previous work. It was hypothesized that the chaotic nature of the flow in the cylinder with aspect ratio 3 might actually accelerate the reaction due to enhanced mixing which could help all the reagents reach the desired temperatures - this prediction was validated by performing PCR experiments, where we observed that while the reaction time was only $10 \mathrm{~min}$ for the cylinder with aspect ratio 3 , the reaction time for the cylinder with aspect ratio 9 was $20 \mathrm{~min}$.

Numerical simulations were also performed by coupling the flow and reaction kinetics by following the motion of a particle on a streamtrace, and it was found that the cylinder with aspect ratio 9 seemed to possess faster reaction rates. However, our experiments strongly contrast this result because our simulations model only a single streamtrace - this is a limitation of our numerical simulation. This could be overcome by performing simulations where the reaction kinetics is integrated into the flow - we found this approach unfeasible using available software packages like FLUENT and COMSOL. 
The chaotic nature of the flow was evaluated both qualitatively and quantitatively through the use of Poincaré maps and correlation dimensions. Using these approaches, we have established the presence of chaos in the cylinder with aspect ratio 3 and the absence of the same in the cylinder with aspect ratio 9. We also computed the correlation dimension for cylinders of varying aspect ratios and volumes - this helps us to map out the chaotic Rayleigh-Bénard convection regime for various aspect ratios, for a particular volume of the cylinder. The Rayleigh-Bénard system was also studied in greater detail by changing the Rayleigh number for constant aspect ratios and by changing the aspect ratio for constant Rayleigh numbers to understand transitions between flows.

Experimental computation of the flow and temperature fields was also performed using particle image velocimetry and laser induced fluorescence. It was found that using glass capillaries for visualization purposes was beneficial because of the optical clarity provided by glass, however, it was also found that using glass created newer problems sealing the fluid inside the cylinder without any air bubbles was very difficult. The effect of the glass sidewalls on the flow inside also cannot be ignored since the numerical simulations were performed for insulated sidewalls. These problems can be overcome by using an acrylic cylinder with a highly polished inner and outer surface. Validation of the numerical simulation was performed using flow visualization from an optical microscope. This method, though rudimentary, proved to be useful to compare against numerical simulations. 
Future research could focus on predicting the effect of correlation dimension on the rate of reaction - this would answer a question on how much chaos is optimal to accelerate the rate of the polymerase chain reaction. Three dimensional PIV and LIF measurements are also needed in order to accurately compute the velocity and temperature fields in the cylinder. 


\section{REFERENCES}

1. Chandrasekhar, S., 1961, Hydrodynamic and Hydromagnetic Stability, Clarendon Press, Oxford, U.K.

2. Charlson, G. S., and Sani, R. L., 1970, "Thermoconvective Instability in a Bounded Cylindrical Fluid Layer," International Journal of Heat and Mass Transfer, 13, pp. 1479-1496.

3. Charlson, G. S., and Sani, R. L., 1971, "On Thermoconvective Instability in a Bounded Cylindrical Fluid Layer," International Journal of Heat and Mass Transfer, 14, pp. 2157-2160.

4. Gershuni, G. Z., and Zhukovitskii, E. M., 1976, Convective Stability of Incompressible Fluids (translated from Russian), Israel Program for Scientific Translations, Jerusalem.

5. Muller, G., Neumann, G., and Weber, W., 1984, "Natural Convection in Vertical Bridgman Configurations,” Journal of Crystal Growth, 70, pp. 78-93.

6. McPherson, M. J., and Møller, S. G., 2000, PCR, BIOS Scientific Publishers Limited, Oxford, U.K.

7. Butler, D., 2006, “Disease Surveillance Needs a Revolution,” Nature, 440, pp. 67.

8. Chin, C. D., Linder, V., and Sia, S. K., 2007, "Lab-on-a-chip Devices for Global Health: Past Studies and Future Opportunities," Lab on a Chip, 7, pp. 41-57. 
9. King, D. A., Peckham, C., Waage, J. K., Brownlie, J., and Woolhouse, M. E. J., 2006, “Infectious Diseases: Preparing for the Future.” Science, 313, pp. 13921393.

10. Sauer, S., Lange, B. M. H., Gobom, J., Nyarsik, L., Seitz, H., and Lehrach, H., 2005, "Miniaturization in Functional Genomics and Proteomics," Nature Reviews: Genetics, 6, pp. 465-476.

11. Syvänen, A. C., 2005, "Toward Genome-wide SNP Genotyping," Nature Genetics, 37, pp. S5-S10.

12. Yager, P., Edwards, T., Fu, E., Helton, K., Nelson, K., Tam, M., and Weigl, B. H., 2006, "Microfluidic Diagnostic Technologies for Global Public Health," Nature, 442, pp. 412-418.

13. Yang, S., and Rothman, R. E., 2004, "PCR-based Diagnostics for Infectious Diseases: Uses, Limitations, and Future Applications in Acute-care Settings," The Lancet: Infectious Diseases, 4, pp. 337-348.

14. Spitzack, K. D., and Ugaz, V. M., 2005, "Polymerase Chain Reaction in Miniaturized Systems: Big Progress in Little Devices," Microfluidic Techniques: Reviews and Protocols, Minteer, S. D., ed., Methods in Molecular Biology Series, Humana Press, Inc., Totowa, New Jersey, pp. 97-129.

15. Krishnan, M., Ugaz, V. M., and Burns, M. A., 2002, "PCR in a Rayleigh-Bénard Convection Cell," Science, 298, pp. 793. 
16. Krishnan, M., Agrawal, N., Burns, M. A., and Ugaz, V. M., 2004, "Reactions and Fluidics in Miniaturized Natural Convection Systems," Analytical Chemistry, 76, pp. 6254-6265.

17. Hennig, M., and Braun, D., 2005, "Convective Polymerase Chain Reaction around Micro Immersion Heater,” Applied Physics Letters, 87, pp. 183901-3.

18. Braun, D., Goddard, N. L., and Libchaber, A., 2003, "Exponential DNA Replication by Laminar Convection,” Physical Review Letters, 91, pp. 1581034.

19. Chung, K. H., Park, S. H., and Choi, Y. H., 2009, “A Palmtop PCR System with a Disposable Polymer Chip Operated by the Thermosiphon Effect," Lab on a Chip, 10, pp. 202-210.

20. Wheeler, E. K., Benett, W., Stratton, P., Richards, J., Chen, A., Christian, A., Ness, K. D., Ortega, J., Li, L. G., Weisgraber, T. H., Goodson, K., and Milanovich, F., 2004, "Convectively Driven Polymerase Chain Reaction Thermal Cycler," Analytical Chemistry, 76, pp. 4011-4016.

21. Chen, Z., Qian, S., Abrams, W. R., Malamud, D., and Bau, H. H., 2004, "Thermosiphon-Based PCR Reactor: Experiment and Modeling," Analytical Chemistry, 76, pp. 3707-3715.

22. Agrawal, N., and Ugaz, V. M., 2007, "A Buoyancy-Driven Compact Thermocycler for Rapid PCR," Clinics in Laboratory Medicene, 27, pp. 215-223. 
23. Agrawal, N., Hassan, Y. A., and Ugaz, V. M., 2007, “A Pocket-Sized Convective PCR Thermocycler," Angewandte Chemie International Edition, 46, pp. 4316 4319.

24. Yao, D.-J., Chen, J.-R., and Ju, W.-T., 2007, "Micro-Rayleigh-Bénard Convection Polymerase Chain Reaction System," Journal of Micro/Nanolithography, 6, pp. 043007.

25. Zhang, C., and Xing, D., 2009, "Parallel DNA Amplification by Convective Polymerase Chain Reaction with Various Annealing Temperatures on a Thermal Gradient Device,” Analytical Biochemistry, 387, pp. 102-112.

26. Kopecka, K., Drouin, G., and Slater, G. W., 2004, "Capillary Electrophoresis Sequencing of Small ssDNA Molecules versus the Ogston Regime: Fitting Data and Interpreting Parameters,” Electrophoresis, 25, pp. 2177-2185.

27. Larson, R. G., 1992, "Instabilities in Viscoelastic Flows," Rheologica Acta, 31, pp. 213-263.

28. Allen, J. W., Kenward, M., and Dorfman, K. D., 2008, “Coupled Flow and Reaction during Natural Convection PCR," Microfluidics and Nanofluidics, 5, pp. $121-130$.

29. Yariv, E., Ben-Dov, G., and Dorfman, K. D., 2005, "Polymerase Chain Reaction in Natural Convection Systems: A Convection-Diffusion-Reaction Model," Europhysics Letters, 71, pp. 1008-1014.

30. Cross, M. C., and Hohenberg, P. C., 1993, "Pattern Formation outside of Equilibrium.” Reviews of Modern Physics, 65, pp. 851-1112. 
31. Davaille, A., 1999, "Simultaneous Generation of Hotspots and Superswells by Convection in a Heterogeneous Planetary Mantle.” Nature, 402, pp. 756-760.

32. Brown, R. A., 1988, “Theory of Transport Processes in Single Crystal Growth from the Melt." American Institute of Chemical Engineering Journal, 34, pp. $881-911$.

33. Kim, H. J., and Beskok, A., 2007, "Quantification of Chaotic Strength and Mixing in a Micro Fluidic System," Journal of Micromechanics and Microengineering, 17, pp. 2197-2210.

34. Grassberger, P., and Procaccia, I., 1983, "Characterization of Strange Attractors," Physical Review Letters, 50, pp. 346-349.

35. Hillborn, R., 2001, Chaos and Nonlinear Dynamics: An Introduction for Scientists and Engineers, Oxford University Press, New York.

36. Toomey, J. P., Kane, D. M., Valling, S., and Lindberg, A. M., 2009, “Automated Correlation Dimension Analysis of Optically Injected Solid State Lasers," Optics Express, 17, pp. 7592-7608.

37. Bucchignani, E., and Stella, F., 1999, "Rayleigh-Bénard Convection in Limited Domains: Part 2- Transition to chaos," Numerical Heat Transfer, Part A: Applications, 36, pp. 17-34.

38. Solomon, T. H., and Gollub, J. P., 1988, "Chaotic Particle Transport in Timedependent Rayleigh-Bénard Convection,” Physical Review A, 38, pp. 62806286. 
39. Solomon, T. H., Tomas, S., and Warner, J. L., 1998, "Chaotic Mixing of Immiscible Impurities in a Two-dimensional Flow," Physics of Fluids, 10, pp. $342-350$.

40. Camassa, R., and Wiggins, S., 1991, "Chaotic Advection in a Rayleigh-Bénard Flow," Physical Review A, 43, pp. 774-797.

41. Fountain, G. O., Khakhar, D. V., and Ottino, J. M., 1998, "Visualization of Three-dimensional Chaos," Science, 281, pp. 683-686.

42. Heslot, F., Castaing, B., and Libchaber, A., 1987, "Transitions to Turbulence in Helium Gas,” Physical Review A, 36, pp. 5870-5873.

43. Lee, T. W., 2008, Thermal and Flow Measurements, Taylor \& Francis Group, CRC Press, New York.

44. Raffel, M., Willert, C. E., Wereley, S. T., and Kompenhans, J., 2007, Particle Image Velocimetry: A Practical Guide (Experimental Fluid Mechanics), Springer, New York.

45. Melling, A., 1997, "Tracer Particles and Seeding for Particle Image Velocimetry," Measurement Science and Technology, 8(12), pp. 1406-1416.

46. Wereley, S. T., and Meinhart, C. D., 2010, "Recent Advances in Micro-Particle Image Velocimetry," Annual Review of Fluid Mechanics, 42, pp. 557-576.

47. Smith, C. R., Sabatino, D. R., and Praisner, T. J., 2001, "Temperature Sensing with Thermochromic Liquid Crystals,” Experiments in Fluids, 30, pp. 190-201. 
48. Sakakibara, J., and Adrian, R. J., 1999, "Whole Field Measurement of Temperature in Water Using Two-Color Laser Induced Fluorescence," Experiments in Fluids, 26, pp. 7-15.

49. Wereley S.T., and Nguyen N.T., 2002, Fundamentals and Applications of Microfluidics, Artech House, Boston. 


\section{VITA}

Name: $\quad$ Radha Malini Gowri Muddu

Address: $\quad 309$, Jack E. Brown Building, Texas A\&M University, College Station, TX 77843-3123

Email Address: radha.m@gmail.com

Education: $\quad$ B.Tech., Mechanical Engineering, Indian Institute of Technology Madras, 2004

M.Tech., Mechanical Engineering, Indian Institute of Technology Madras, 2005

M.S., Mechanical Engineering, Purdue University, 2007 